COMMUNICATIONS IN

ANALYSIS AND GEOMETRY

Volume 5, Number 4, 625-684, 1997

\title{
The first two Betti numbers of the moduli spaces of vector bundles on surfaces
}

\author{
JUN LI ${ }^{1}$
}

\section{Introduction.}

This paper is a continuation of our effort in understanding the geometry of the moduli space of stable vector bundles. For any polarized smooth projective surface $(X, H)$ and any choice of $(I, d) \in \operatorname{Pic}(X) \times H^{4}(X, \mathbb{Z})$, there is a coarse moduli space $\mathfrak{M}(I, d)^{0}$ of rank two $\mu$-stable (with respect to $H$ ) locally free sheaves $\mathcal{E}$ of $\wedge^{2} \mathcal{E} \cong I$ and $c_{2}(\mathcal{E})=d$. This moduli space has been studied extensively recently. One important discovery is that the moduli space $\mathfrak{M}(I, d)^{0}$ exhibits remarkable properties at stable range. To cite a few, for arbitrary surface the moduli space $\mathfrak{M}(I, d)^{0}$ has the expected dimension, is smooth at general points and is irreducible, and for a large class of surfaces of general type $\mathfrak{M}(I, d)^{0}$ are of general type, all true for $d$ sufficiently large [Fr, GL, Li2, Do, Zh]. In this paper, we will investigate another aspect of this moduli space. Namely, the Betti numbers of $\mathfrak{M}(I, d)^{0}$. So far, there have been a lot of progress along this direction based on two different approaches: Algebro-geometric approach and gauge theoretic approach. The algebraic geometry approach is relatively new. In [ES, Ki,Yo], they studied in detail the Betti numbers of the moduli space of stable sheaves over $\mathbf{P}^{2}$ (for the rank two and higher rank cases). Beauville [Be] has a nice observation concerning some rational surfaces and Göttsche and Huybrechts [GH] have worked out the case for K3 surfaces. The gauge theory approach has been around for quite a while. To begin with, let $(M, g)$ be a compact oriented Riemannian four-manifold and let $P_{d}$ be a smooth $\mathrm{SO}(3)$ (or SU(2))-bundle over $M$ whose associated vector bundle has rank $2, c_{1}=I$ and $c_{2}=d$. Consider the pair

$$
\mathcal{N}\left(P_{d}\right) \subset \mathcal{B}\left(P_{d}\right)^{*},
$$

where $\mathcal{B}\left(P_{d}\right)^{*}$ is the space of gauge equivalent classes of irreducible connections on $P_{d}$ and $\mathcal{N}\left(P_{d}\right)$ is the subspace of Anti-Self-Dual connections. By a

\footnotetext{
${ }^{1}$ This research was partially supported by NSF grant DMS-9307892 and Alfred P. Sloan research fellowship.
} 
celebrated theorem of Donaldson, when $M=X$ is an algebraic surface with a Kahler metric associated to the ample divisor $H, \mathfrak{M}(I, d)^{0}$ is canonically isomorphic to $\mathcal{N}\left(P_{d}\right)$. The advantage of looking at the pair $(0.1)$ is that $H_{*}\left(\mathcal{B}\left(P_{d}\right)^{*}\right)$ is calculable, at least modulo torsions, in terms of the homotopy type of $X$. Thus we will know $H_{*}\left(\mathfrak{M}(I, d)^{0}\right)$ if we know the induced homomorphism

$$
\ell(d)_{i}: H_{i}\left(\mathfrak{M}(I, d)^{0}, \mathbb{Z}\right) \longrightarrow H_{i}\left(\mathcal{B}\left(P_{d}\right)^{*}, \mathbb{Z}\right) .
$$

In [AJ], Atiyah and Jones conjectured that for $M=S^{4}$ and SU(2)-bundle $P_{d}$, there is a sequence of (explicit) integers $\left\{q_{k}\right\}$ such that for $d \geq q_{k},(0.2)$ is an isomorphism for $i \leq k$. Later, Taubes' work [Ta] suggests that similar conjecture should hold for arbitrary 4-manifold with possibly different sequence $\left\{q_{k}\right\}$. This conjecture has been confirmed for $S^{4}, \mathbf{C F}^{2}$ and $K 3$ surfaces, see [HB, $\mathrm{HBM}^{2}, \mathrm{ES}, \mathrm{GH}, \mathrm{Ki}$, Ti1, Ti2, Yo].

In this paper, we will study $H_{*}\left(\mathfrak{M}(I, d)^{0}\right)$ for arbitrary algebraic surface. Due to technical difficulties, we are unable to prove the generalized AtiyahJones conjecture for all Betti numbers. Instead, we will prove the following theorems that will determine the first two Betti numbers of the moduli space.

Theorem 0.1. For any smooth projective surface $(X, H)$ and any $I \in$ $\operatorname{Pic}(X)$, there is an integer $N$ depending on $(X, I, H)$ so that whenever $d \geq N$, then

$$
\ell(d)_{i}: H_{i}\left(\mathfrak{M}(I, d)^{0} ; \mathbb{Q}\right) \longrightarrow H_{i}\left(\mathcal{B}\left(P_{d}\right)^{*} ; \mathbb{Q}\right)
$$

is an isomorphism for $i \leq 2$, where $P_{d}$ is the $S O(3)$ (or $S U(2)$ )-buindle associated to the rank two complex vector bundle $E$ with $\wedge^{2} E \cong I$ and $c_{2}(E)=d$.

By calculating the first and second Betti numbers of $\mathcal{B}\left(P_{d}\right)^{*}$, we get

Theorem 0.2. With the notation as in theorem 0.1, then there is an integer $N$ depending on $(X, I, H)$ so that for $d \geq N$, $\operatorname{dim} H_{1}\left(\mathfrak{M}(I, d)^{0}\right)$ and $\operatorname{dim} H_{2}\left(\mathfrak{M}(I, d)^{0}\right)$ are $b_{1}$ and $b_{2}+\frac{1}{2} b_{1}\left(b_{1}-1\right)$ respectively, where $b_{i}=$ $\operatorname{dim} H_{i}(X)$.

In algebraic geometry, there is a moduli space $\mathfrak{M}(I, d)$ of $H$-stable rank two sheaves $\mathcal{E}$ with $\operatorname{det} \mathcal{E} \cong I$ and $c_{2}(\mathcal{E})=d . \mathfrak{M}(I, d)$ is quasi-projective and contains $\mathfrak{M}(I, d)^{0}$ as a Zariski open subset. We calculate the first two Betti numbers of $\mathfrak{M}(I, d)$ as well. 
Theorem 0.3. With the notation as in theorem 0.1, then there is an integer $N$ depending on $(X, I, H)$ so that for $d \geq N, \operatorname{dim} H_{1}(\mathfrak{M}(I, d))$ and $\operatorname{dim} H_{2}(\mathfrak{M}(I, d))$ are $b_{1}$ and $b_{2}+\frac{1}{2} b_{1}\left(b_{1}-1\right)+1$ respectively.

There is a general principle [Mu] that explains why the Betti numbers of $\mathfrak{M}(I, d)$ take the form in theorem 0.3. For simplicity, let us assume $\mathfrak{M}(I, d)$ is projective and admits a universal family, say $\mathcal{E}$ over $X \times \mathfrak{M}(I, d)$. Then $\mathcal{E}$ is expected to contain all information of $\mathfrak{M}(I, d)$. For instance, the cohomology ring $H^{*}(\mathfrak{M}(I, d)$ ) (with rational coefficient) should be generated by the Kunneth components of $c_{i}(\mathcal{E})$. Put it differently, each $c_{i}(\mathcal{E})$ defines homomorphisms

$$
\mu_{i}^{[*]}: H_{*}(X) \longrightarrow H^{2 i-*}(\mathfrak{M}(I, d))
$$

via slant product. Then the Mumford principle states that $H^{*}(\mathfrak{M}(I, d))$ is generated by the image of $\left\{\mu_{i}\right\}_{i \geq 2}$, and that within range up to $r(d)$ $(r(d) \rightarrow \infty$ when $d \rightarrow \infty)$ their images obey no restraint other than the obvious commutativity law of the cohomology ring. In particular, if we look at $H^{1}(\mathfrak{M}(I, d))$, then it should be generated (freely) by the images of $\mu_{2}^{[3]}: H_{3}(X) \rightarrow H^{1}(\mathfrak{M}(I, d))$, which has dimension $b_{1}$ by Poincare duality. For $H^{2}(\mathfrak{M}(I, d))$, it should be generated freely by the wedge product of $H^{1}(\mathfrak{M}(I, d))$, the image of $\mu_{2}^{[2]}: H_{2}(X) \rightarrow H^{2}(\mathfrak{M}(I, d))$ and the image of $\mu_{3}^{[4]}: H_{4}(X) \rightarrow H^{2}(\mathfrak{M}(I, d))$. Together they span a linear space of total dimension $b_{2}+\frac{1}{2} b_{1}\left(b_{1}-1\right)+1$.

One motivation of the current work is to determine the Picard group of the moduli space $\mathfrak{M}(I, d)^{0}$ and $\mathfrak{M}(I, d)$. As is known, $\operatorname{Pic}(\mathfrak{M}(I, d))$ is largely determined by $\operatorname{dim} H_{1}(\mathfrak{M}(I, d))$ and $\operatorname{dim} H_{2}(\mathfrak{M}(I, d))$. In [Li3], we have determined their Picard groups based on the information gained here.

Now we explain the strategy in establishing these Theorems. In the following, we let $i=1$ or 2 . According to Taubes [Ta], for large $d$ there are canonical homomorphisms $\tau(d)_{i}$ and $\tilde{\tau}(d)_{i}$ making the following diagram commutative: ${ }^{2}$

$$
\begin{array}{ccc}
H_{i}\left(\mathfrak{M}(I, d)^{0}\right) & \stackrel{\ell(d)_{i}}{\longrightarrow} & H_{i}\left(\mathcal{B}\left(P_{d}\right)^{*}\right) \\
\left\lfloor\tau(d)_{i}\right. & & \downarrow \tilde{\tau}(d)_{i} \\
H_{i}\left(\mathfrak{M}(I, d+1)^{0}\right) \stackrel{\ell(d+1)_{i}}{\longrightarrow} & H_{i}\left(\mathcal{B}\left(P_{d+1}\right)^{*}\right) .
\end{array}
$$

\footnotetext{
${ }^{2}$ Taubes constructed a diagram using the space of based connections. His diagram is identical to ours in case $i \leq 2$ because $\mathrm{SO}(3)$ and $\mathrm{SU}(2)$ are rational 3-sphere.
} 
(Here and in the remainder of this paper, all homologies are with rational coefficients unless otherwise is stated.) Further, $\tilde{\tau}(d)_{i}$ is an isomorphism, $\ell(d)_{i}$ is surjective for sufficiently large $d$ and the composition of $\tau(\cdot)_{i}$ 's

$$
\tau(d, d+k)_{i}: H_{i}\left(\mathfrak{M}(I, d)^{0}\right) \rightarrow H_{i}\left(\mathfrak{M}(I, d+k)^{0}\right)
$$

has the property that

$$
\operatorname{ker}\left\{\ell(d)_{i}\right\} \subset \operatorname{ker}\left\{\tau(d, d+k(d))_{i}\right\}
$$

for some $k(d)$. Thus, if for large $d$ the homomorphism $\tau(d)_{i}$ is surjective, $\tau(d)_{i}$ must be an isomorphism for sufficiently large $d$. Therefore by (0.4), $\ell(d)_{i}$ will be an isomorphism for sufficiently large $d$ as well, thus establishing theorem (0.1). (Please see section 4 for more details.)

The homomorphism

$$
\tau(d)_{i}: H_{i}\left(\mathfrak{M}(I, d)^{0}\right) \longrightarrow H_{i}\left(\mathfrak{M}(I, d+1)^{0}\right), \quad i \leq 2
$$

can be defined easily in our context. Set $\mathfrak{M}(I, d)^{\mu} \subset \mathfrak{M}(I, d)$ be the open subset of $\mu$-stable sheaves. We fix an $x \in X$ and let $\mathcal{S}_{1}^{x} \mathfrak{M}(I, d+1)^{\mu} \subset$ $\mathfrak{M}(I, d+1)^{\mu}$ be those $\mathcal{E}$ such that $\mathcal{E}^{\vee \vee} / \mathcal{E} \cong \mathbb{C}_{x} . \mathcal{S}_{1}^{x} \mathfrak{M}(I, d+1)^{\mu}$ is a $\mathbf{P}^{1}$ bundle over $\mathfrak{M}(I, d)^{\mu}$ by sending $\mathcal{E}$ to $\mathcal{E}^{\vee \vee}$. Let $V_{0}$ be a general fiber. Then the inclusion $V_{0} \subset \mathfrak{M}(I, d+1)^{\mu}$ and the bundle $\mathcal{S}_{1}^{x} \mathfrak{M}(I, d+1)^{\mu} \rightarrow \mathfrak{M}(I, d)^{0}$ induce a commutative diagram

$$
\begin{aligned}
& 0 \rightarrow H_{i}\left(V_{0}\right) \rightarrow H_{i}\left(\mathcal{S}_{1}^{x} \mathfrak{M}(I, d+1)^{\mu}\right) \rightarrow H_{i}\left(\mathfrak{M}(I, d)^{0}\right) \quad \rightarrow 0 \\
& \| r(d)_{i} \downarrow \\
& 0 \rightarrow H_{i}\left(V_{0}\right) \rightarrow H_{i}\left(\mathfrak{M}(I, d+1)^{\mu}\right) \leftarrow H_{i}\left(\mathfrak{M}(I, d+1)^{0}\right) .
\end{aligned}
$$

Let $i$ be 1 or 2 . When $d$ is sufficiently large, $H_{i}\left(\mathfrak{M}(I, d+1)^{\mu}\right)$ is a direct sum of the images of $H_{i}\left(V_{0}\right)$ and $H_{i}\left(\mathfrak{M}(I, d+1)^{0}\right)$. Therefore, (0.6) induces a homomorphism $H_{i}\left(\mathfrak{M}(I, d)^{0}\right) \rightarrow H_{i}\left(\mathfrak{M}(I, d+1)^{0}\right)$ that is the mentioned homomorphism $\tau(d)_{i}$. (See Lemma 4.2 for details.)

From (0.6), $\tau(d)_{i}$ is surjective if $r(d)_{i}$ is surjective. In this paper, we will prove the surjectivity of $r(d)_{i}$ by establishing the following two theorems:

Theorem 0.4. With the notation as in theorem 0.1, then there is an integer $N$ so that whenever $d \geq N$, then for $i=1,2$,

$$
H_{i}(\mathcal{S} \mathfrak{M}(I, d)) \longrightarrow H_{i}(\mathfrak{M}(I, d)),
$$

is surjective, where $\mathcal{S} \mathfrak{M}(I, d)=\mathfrak{M}(I, d)-\mathfrak{M}(I, d)^{0}$. 
Theorem 0.5. With the notation as in theorem 0.1, then there is an integer $N$ so that whenever $d \geq N$, then for $i=1,2$ and closed $x \in X$, the image of

$$
H_{i}(\mathcal{S M}(I, d)) \longrightarrow H_{i}(\mathfrak{M}(I, d))
$$

is contained in the image of

$$
H_{i}\left(\mathcal{S}_{1}^{x} \mathfrak{M}(I, d)^{\mu}\right) \longrightarrow H_{i}(\mathfrak{M}(I, d)) .
$$

Clearly, Theorem 0.4 and 0.5 together implies the surjectivity of $r(d)_{i}$. The strategy to establish theorem 0.4 is to apply the Lefschetz hyperplane theorem to the moduli space. The classical Lefschetz hyperplane theorem states that for any smooth, projective variety $Z$ of complex dimension $n$ and any smooth very ample divisor $Z_{1} \subset Z$, the pair $\left(Z, Z_{1}\right)$ has vanishing homology groups up to degree $n-1$. Concerning our situation, the ideal pair to look at is $(\mathfrak{M}(I, d), \mathcal{S M}(I, d))$. But Lefschetz hyperplane theorem does not apply directly to this pair because $\mathcal{S M}(I, d)$ is definitely not ample. Instead, we will first find an ample subvariety $\mathcal{Y}$ of $\mathfrak{M}(I, d)$ and apply the generalized Lefschetz hyperplane theorem to the pair $(\mathfrak{M}(I, d), \mathcal{Y})$ to establish the surjectivity of

$$
H_{i}(\mathcal{Y}, \mathcal{S M}(I, d) \cap \mathcal{Y}) \longrightarrow H_{i}(\mathfrak{M}(I, d), \mathcal{S M}(I, d)), \quad i \leq 2 .
$$

The set $\mathcal{Y}$ has an explicit geometric description: let $C \in|n H|$ be a fixed smooth divisor for some $n>0$. Then $\mathcal{Y} \subset \mathfrak{M}(I, d)$ consists of those $\mathcal{E}$ such that $\mathcal{E}_{\mid C}$ is not semistable, including those where $\mathcal{E}_{\mid C}$ are not locally free. By work of [Li1], there is a morphism

$$
\Psi_{C}: \mathfrak{M}(I, d) \longrightarrow \mathbf{P}^{M}
$$

and a codimension $3 g(C)-2$ linear subspace $V \subset \mathbf{P}^{N}$ such that $\Phi_{C}^{-1}(V)=\mathcal{Y}$. Hence we obtain the surjectivity of (0.7) by applying the stratified Morse theory developed in [GM] to $\Psi_{C}$ and $V \subset \mathbf{P}^{N}$.

The next step is to show that

$$
H_{i}(\mathcal{Y}, \mathcal{S} \mathfrak{M}(I, d) \cap \mathcal{Y}), \quad i \leq 2
$$

is trivial. The tactic is to construct explicitly a homology between any class in (0.9) with the null class by exploiting the fact that restriction to $C$ of sheaves in $\mathcal{Y}$ are not semistable. Here is an outline: for any locally free sheaf $\mathcal{E} \in \mathcal{Y}$, let $\mathcal{L}$ be the destabilizing quotient sheaf of $\mathcal{E}_{\mid C}$ and let $\mathcal{F}$ be the elementary transformation of $\mathcal{E}$ defined by the exact sequence

$$
0 \longrightarrow \mathcal{F} \longrightarrow \mathcal{E} \longrightarrow \mathcal{L} \longrightarrow 0 .
$$


Then $\mathcal{E}$ can be reconstructed from $\mathcal{F}$ via

$$
0 \longrightarrow \mathcal{E} \longrightarrow \mathcal{F}(C) \stackrel{\alpha}{\longrightarrow} \mathcal{O}(I) \otimes \mathcal{L}^{-1} \longrightarrow 0 .
$$

If we vary $\alpha$ and $\mathcal{L}$, we get deformations of $\mathcal{E}$ within $\mathcal{Y}$. In certain cases, we can deform $\mathcal{E}$ to non-locally free sheaves this way. This method was used by O'Grady in showing that $H_{0}(\mathcal{Y}, \mathcal{S M}(I, d) \cap \mathcal{Y})=0$ [OG1]. In this paper, we will work out this construction in the relative case to prove the vanishing of (0.9).

A large portion of the current work is devoted to study the singularities of various sets. This is necessary because generalized Lefschetz hyperplane theorem only apply to varieties with "mild" singularities. In principle, the current approach should work for all homology groups through a range that depends on the (local) topology of the singularities of $\mathcal{Y}$. For the moment, the author can only show that $\mathcal{Y}$ is locally irreducible away from $\mathcal{S M}_{H}(I, d)$. Nevertheless, the local irreducibility of $\mathcal{Y}$ is sufficient to show the vanishing of $(0.9)$ and thus establishing theorem 0.4 . Theorem 0.5 is proved by carefully studying the inclusion $\mathcal{S} \mathfrak{M}(I, d) \subset \mathfrak{M}(I, d)$.

The layout of the paper is as follows: In $\S 1$, we will gather all relevant properties of the moduli space $\mathfrak{M}(I, d)$ of which we will need. These include discussion of singularities of algebraic sets. In $\S 2$, by studying deformation of sheaves over curves, we will show that the set $\mathcal{Y} \subset \mathfrak{M}(I, d)$ is locally irreducible away from $\mathcal{S} \mathfrak{M}_{H}(I, d)$. 33 is a refinement of [La, OG1] in which we will demonstrate how one can deform a family of locally free sheaves to non-locally free sheaves and thus deriving the vanishing of (0.9). The theorem 0.4 and 0.5 will be proved in $\S 5$. Most of the materials concerning singularity and Lefschetz hyperplane theorem are drawn from the book of Goresky and MacPherson [GM].

\section{Preliminaries.}

In the first part of this section, we will gather results of $\mathfrak{M}(I, d)$ that are important to our study. Some of them have already appeared or: known to the experts and others are improvements of the earlier results. We will give the reference to each result and provide proof if necessary.

First, let us introduce the convention that will be used throughout this paper. In this paper, all schemes considered are of finite type and are over complex number field. All points of schemes are closed points. We will use Zariski topology throughout the paper unless otherwise mentioned. Thus a closed subset is a union of finite closed subvarieties. We will use algebraic 
subset to mean finite union of locally closed subsets. By dimension of an algebraic set we mean complex dimension. We will only consider coherent sheaves in this paper and will not distinguish a vector bundle from the sheaf of its sections. In studying homology, we will use the notion of chains and cycles. An $r$-chain in $Z$ is a closed real $r$-dimensional piecewise smooth subset with or without boundary and an $r$-cycle is an $r$-chain without boundary. We will use $\partial \Sigma$ to denote the boundary of a chain $\Sigma$. When $\partial \Sigma=0$, then we will use $[\Sigma]$ to denote the corresponding homology class.

Throughout this paper, we fix a smooth algebraic surface $X$ and a line bundle $I \in \operatorname{Pic}(X)$. Let $H$ be an ample divisor on $X$. We say a rank two sheaf $\mathcal{E}$ is $H$-stable (resp. $H$-semistable) if for any proper quotient sheaf $\mathcal{E} \rightarrow \mathcal{F}$, (i.e. it has non-trivial kernel) we have

$$
\frac{1}{\operatorname{rank\mathcal {E}}} \chi_{\mathcal{E}}(n)<\frac{1}{\operatorname{rank} \mathcal{F}} \chi_{\mathcal{F}}(n) \quad(\text { resp. } \leq)
$$

for sufficiently large $n$, where $\chi_{\mathcal{E}}(n)=\chi\left(\mathcal{E} \otimes H^{\otimes n}\right)$ is the value of the Hilbert polynomial of $\mathcal{E}$. Note that $H$-semistable sheaves are necessarily torsion free. Similarly, we say a torsion free $\operatorname{sheaf} \mathcal{E}$ is $H$ - $\mu$-stable (resp. $H$ - $\mu$-semistable ) if for any rank one torsion free quotient sheaf $\mathcal{E} \rightarrow \mathcal{F}$, we have $\mu(\mathcal{E})<\mu(\mathcal{F})$ (resp. $\leq$ ), where $\mu(\mathcal{E})=\frac{1}{\operatorname{rank\mathcal {E}}} c_{1}(\mathcal{E}) \cdot H$. We define stable and $\mu$-stable sheaves on curves similarly. We also need the notion of $e$-stable. For any constant $e$, a rank two sheaf $\mathcal{E}$ is said to be $e$-stable if for any rank one torsion free quotient sheaf $\mathcal{E} \rightarrow \mathcal{F}$, we have $\mu(\mathcal{E})<\mu(\mathcal{F})+e$. One notices that $H$ $\mu$-stable implies $H$-stable and $H$-semistable implies $H$ - $\mu$-semistable. In case the choice of $H$ is apparent from the context, we will simply call them stable or $\mu$-stable. We agree that by unstable we mean not semistable. According to [Gi], for any $d \in \mathbb{Z}$ there is a moduli scheme $\overline{\mathfrak{M}}_{H}(I, d)$ of rank two $H$ semistable sheaves $\mathcal{E}$ with $\operatorname{det} \mathcal{E}=I$ and $c_{2}(\mathcal{E})=d$ (modulo equivalence relation). $\overline{\mathfrak{M}}_{H}(I, d)$ is projective. In the following, we will freely refer a semistable sheaf $\mathcal{E}$ as a point in $\overline{\mathfrak{M}}_{H}(I, d)$.

There are several open subsets of $\overline{\mathfrak{M}}_{H}(I, d)$ that are relevant to our study. The first collection consists of open subset $\mathfrak{M}_{H}(I, d) \subset \overline{\mathfrak{M}}_{H}(I, d)$ of all $H$-stable sheaves (called the moduli of stable sheaves), $\mathfrak{M}_{H}(I, d)^{\mu} \subset$ $\mathfrak{M}_{H}(I, d)$ of all $\mu$-stable sheaves and $\mathfrak{M}_{H}(I, d)^{0} \subset \mathfrak{M}_{H}(I, d)$ of all $\mu$ stable locally free sheaves. In most cases, $\mathfrak{M}_{H}(I, d)^{0}$ is a Zariski dense open subset of $\overline{\mathfrak{M}}_{H}(I, d)$. We let $\mathcal{S} \overline{\mathfrak{M}}_{H}(I, d)=\overline{\mathfrak{M}}_{H}(I, d)-\mathfrak{M}_{H}(I, d)^{0}$ and $\mathcal{S M}_{H}(I, d)=\mathfrak{M}_{H}(I, d)-\mathfrak{M}_{H}(I, d)^{0} . \mathcal{S} \overline{\mathfrak{M}}_{H}(I, d)$ contains (a priori) some points parameterizing locally free sheaves. For integer $l \geq 1$, we let $\mathcal{S}_{l} \mathfrak{M}_{H}(I, d) \subset \mathcal{S} \mathfrak{M}_{H}(I, d)$ be the set of non-locally free sheaves $\mathcal{E}$ such that the length $\ell\left(\mathcal{E}^{\vee \vee} / \mathcal{E}\right)=l$, where $\mathcal{E}^{\vee \vee}$ is the double dual of $\mathcal{E}$, and let 
$\mathcal{S}_{l}^{0} \mathfrak{M}_{H}(I, d) \subset \mathcal{S}_{l} \mathfrak{M}_{H}(I, d)$ be the subset of those $\mathcal{E}$ such that $\mathcal{E}^{\vee \vee} / \mathcal{E}$ supports on $l$ distinct points. Note that $\mathcal{S}_{l}^{0} \mathfrak{M}_{H}(I, d) \subset \mathcal{S}_{l} \mathfrak{M}_{H}(I, d)$ is open.

Usually, the algebraic subset $\mathcal{S} \overline{\mathfrak{M}}_{H}(I, d) \subset \overline{\mathfrak{M}}_{H}(I, d)$ is not Cartier (Cartier means that set-theoretically it is locally definable by one equation) which makes the study of the topology difficult. However, in some cases they do. Namely, when $H$ is $(I, d)$-generic.

Definition 1.1. 1. An ample divisor $H$ is called $(I, d)$-generic if for any strictly $H$-semistable sheaf $\mathcal{E}$ with $\operatorname{det} \mathcal{E}=I$ and $c_{2}(\mathcal{E}) \leq d, \mathcal{E}$ is S-equivalent to a direct sum (of rank one sheaves) $\mathcal{L}_{1} \oplus \mathcal{L}_{2}$ such that $c_{1}\left(\mathcal{L}_{1}\right)=c_{1}\left(\mathcal{L}_{2}\right) \in$ $H^{2}(X, \mathbb{R})$.

2. Let $H_{0}$ be any ample divisor. An ample divisor $H$ is called $\left(H_{0}, I, d\right)$ suitable if not only $H$ is $(d, I)$-generic but also has the properity that any $H$-semistable sheaves $\mathcal{E}$ with $\operatorname{det} \mathcal{E}=I$ and $c_{2}(\mathcal{E}) \leq d$ are necessarily $H_{0^{-}} \mu$ semistable.

As was mentioned, the study of the topology of $\mathfrak{M}_{H}(I, d)$ simplifies if $\mathcal{S} \overline{\mathfrak{M}}_{H}(I, d) \subset \overline{\mathfrak{M}}_{H}(I, d)$ is Cartier. Thus we need to choose $H$ that is $(d, I)$-generic. However, in proving the main theorems we need to work on $\mathfrak{M}_{H}(I, d)$ inductively on $d$. Although for fixed $(I, d)$ there are plenty of $(I, d)$-generic ample divisors, each polarization $H$ will cease to be $(I, d)$ generic for large enough $d$, assuming $\operatorname{dim} H^{1,1}(X)>1$. Thus we need to adjust $H$ constantly as $d$ increases. To get by this, we will work: with a set of polarizations simultaneously.

To this end, some discussion on the selection of polarizations is in order. First, any two ample divisors $H_{1}$ and $H_{2}$ will give rise to (canonically) isomorphic moduli spaces $\mathfrak{M}_{H_{1}}(I, d)$ and $\mathfrak{M}_{H_{2}}(I, d)$ if $c_{1}\left(H_{1}\right)$ and $c_{1}\left(H_{2}\right)$ lie on the same (real) line in $H^{2}(X ; \mathbb{R})$. Now let

$$
\mathrm{NS}_{\mathbb{R}}=\left(H^{1,1}(X, \mathbb{R}) \cap H^{2}(X, \mathbb{Q})\right) \otimes_{\mathbb{Q}} \mathbb{R},
$$

let $\mathrm{NS}_{\mathbb{R}}^{+}$be the ample cone and let $\mathrm{NS}_{\mathbb{Q}}$ and $\mathrm{NS}_{\mathbb{Q}}^{+}$be the intersection with $H^{2}(X, \mathbb{Q})$ of the corresponding spaces. For any $\xi \in \mathrm{NS}_{\mathbb{Q}}^{+}$, we define the moduli space $\overline{\mathfrak{M}}_{\xi}(I, d)$ to be $\overline{\mathfrak{M}}_{H}(I, d)$ for some ample $H$ such that $c_{1}(H)=$ $n \xi$ for some $n$. By abuse of notation, in the following we will use $H \in \mathrm{NS}_{\mathbb{Q}}^{+}$ to mean $H$ a $\mathbb{Q}$-divisor with $c_{1}(H) \in \mathrm{NS}_{\mathbb{Q}}^{+}$. Next, let $H_{0}$ be any ample divisor and let $\mathcal{C}_{\varepsilon} \subset \mathrm{NS}_{\mathbb{Q}}$ be an $\varepsilon$-ball in $\mathrm{NS}_{\mathbb{Q}}$ centered at $H_{0} \in \mathrm{NS}_{\mathbb{Q}}^{+}$, after fixing an Euclidean metric on $\mathrm{NS}_{\mathbb{Q}}$. For sufficiently small $\varepsilon>0$, the closure $\operatorname{cl}\left(\mathcal{C}_{\varepsilon}\right)$ of $\mathcal{C}_{\varepsilon}$ in $\mathrm{NS}_{\mathbb{R}}$ is still contained in $\mathrm{NS}_{\mathbb{R}}^{+}$. We call such $\mathcal{C}_{\varepsilon}$ precompact neighborhood of $H_{0} \in \mathrm{NS}_{\mathbb{Q}}^{+}$and denoted by $\mathcal{C}_{\varepsilon} \Subset \mathrm{NS}_{\mathbb{Q}}^{+}$. 
Lemma 1.2. Let $H_{0}$ be an ample line bundle and let $\mathcal{C} \Subset N S_{\mathbb{Q}}^{+}$be a precompact neighborhood of $H_{0} \in N S_{\mathbb{Q}}^{+}$. Then for any choice of $(I, d)$, we can find an $\left(H_{0}, I, d\right)$-suitable $\mathbb{Q}$-ample divisor $H$ in $\mathcal{C}$.

Proof. It follows from theorem 1 on page 398 of [Qi] and the Hodge index theorem.

From now on, we fix an $H_{0} \in \mathrm{NS}_{\mathbb{Q}}^{+}$and a precompact neighborhood $\mathcal{C} \Subset \mathrm{NS}_{\mathbb{Q}}^{+}$of $H_{0} \in \mathrm{NS}_{\mathbb{Q}}^{+}$. We will study moduli space $\mathfrak{M}_{H}(I, d)$ with $H$ an arbitrary $\mathbb{Q}$-divisor in $\mathcal{C}$ and derive estimate that depend on the set $\mathcal{C} \Subset \mathrm{NS}_{\mathbb{Q}}^{+}$rather than individual $H \in \mathcal{C}$. We choose once and for all a smooth $C \in\left|n_{0} H\right|$, where $n_{0}$ is a large even integer satisfying

$$
n_{0}^{2} H^{2}-n_{0}\left|K_{X} \cdot H\right|-n_{0} I \cdot H \geq 10,
$$

and denote by $g$ the genus of $C$ in the remainder of this paper.

Let $H \in \mathcal{C}$. Since usually the moduli space $\mathfrak{M}_{H}(I, d)$ is singular, it is convenient to work with a smooth subset of it:

$$
\mathcal{M}_{d}=\left\{\mathcal{E} \in \mathfrak{M}_{H}(I, d) \mid \mathcal{E} \text { is } \mu \text {-stable and } H^{2}\left(\mathcal{E} n d^{0}\left(\mathcal{E}^{\vee}\right)\right)=0\right\} .
$$

(For any sheaf $\mathcal{E}$, we let $\mathcal{E} n d^{0}(\mathcal{E})$ be the sheaf of traceless endomorphisms of $\mathcal{E}$ and let $\operatorname{Ext}^{i}(\mathcal{E}, \mathcal{E})^{0}$ be the trace-less part of $\operatorname{Ext}^{i}(\mathcal{E}, \mathcal{E})$.) $\mathcal{M}_{d}$ is smooth. We let $\mathcal{M}_{d}^{0}, \mathcal{S M}_{d}$ and $\mathcal{S}_{l} \mathcal{M}_{d}$ etc. be $\mathcal{M}_{d} \cap \mathfrak{M}_{H}(I, d)^{0}, \mathcal{M}_{d} \cap \mathcal{S} \mathfrak{M}_{H}(I, d)$ and $\mathcal{M}_{d} \cap \mathcal{S}_{l} \mathfrak{M}_{H}(I, d)$ etc. respectively. For any constant $e<0$, we let $\overline{\mathfrak{M}}_{H}(I, d)_{e} \subset \overline{\mathfrak{M}}_{H}(I, d)$ be the set of all $e$-stable sheaves. We summarize some properties of these sets in the following lemma.

Lemma 1.3. There is an $N$ depending on $(X, I, \mathcal{C})$ so that whenever $d \geq$ $N$, then for any $H \in \mathcal{C}$,

(1) $\overline{\mathfrak{M}}_{H}(I, d)$ is normal, irreducible and has pure dimension $4 d-I^{2}-$ $3 \chi\left(\mathcal{O}_{X}\right)$;

(2) $\mathfrak{M}_{H}(I, d)$ is a local complete intersection scheme;

(3) Both $\mathcal{M}_{d}$ and $\mathcal{S}_{1} \mathcal{M}_{d}$ are smooth;

(4) $\mathfrak{M}_{H}(I, d)^{0} \subset \overline{\mathfrak{M}}_{H}(I, d), \mathfrak{M}_{H}(I, d) \subset \overline{\mathfrak{M}}_{H}(I, d)$ and $\mathcal{M}_{d} \subset \mathfrak{M}_{H}(I, d)$ are dense. Furthermore, the codimension of the sets $\overline{\mathfrak{M}}_{H}(I, d)-$ $\mathfrak{M}_{H}(I, d)_{e}$ and $\overline{\mathfrak{M}}_{H}(I, d)-\mathcal{M}_{d}$ in $\overline{\mathfrak{M}}_{H}(I, d)$ are at least $10 \mathrm{~g}$, where $e=-2 n_{0}^{2} H^{2}$; 
(5) $\mathcal{S}_{l}^{0} \mathfrak{M}_{H}(I, d)$ has dimension $\operatorname{dim} \mathfrak{M}_{H}(I, d)-l$ and is dense in $\mathcal{S}_{l} \mathfrak{M}_{H}(I, d)$ for $l \leq 10$

(6) There are $B \subset \mathfrak{M}_{H}(I, d)$ and $B_{0} \subset \mathfrak{M}_{H_{0}}(I, d)$, each has codimension at least $-3 \chi\left(\mathcal{O}_{2 C}\right)+2 g+14$, such that

$$
\mathfrak{M}_{H}(I, d)-B \cong \mathfrak{M}_{H_{0}}(I, d)-B_{0},
$$

where each consists of sheaves that are both $H$ and $H_{0}$ stable sheaves.

Proof. (1) and (2) were proved in [GL, Li2]. (3) follows from [At] and (4) and (5) can be found in [Do, Fr, Li1, Qi, Zu]. (6) is proved in [Qi].

We now introduce some subsets of $\mathfrak{M}_{H}(I, d)$ associated to $C$. Let $2 C \subset$ $X$ be the obvious non-reduced subscheme supported on $C$. We define

(1.3) $\Lambda_{1}^{C}=\left\{\mathcal{E} \in \mathfrak{M}_{H}(I, d) \mid \operatorname{Ext}_{X}^{2}\left(\mathcal{E}^{\vee}, \mathcal{E}^{\vee}(-2 C)\right)^{0} \neq 0\right\}$

(1.4) $\Lambda_{2}^{C}=\left\{\mathcal{E} \in \mathfrak{M}_{H}(I, d) \mid \mathcal{E}_{\mid C}\right.$ is locally free and $\operatorname{Ext}^{0}\left(\mathcal{E}_{2 C}, \mathcal{E}_{\mid 2 C}\right)^{0}>4 g+$ $12\}$ and

(1.5) $\Lambda_{\mathcal{E}}^{C}=\left\{\mathcal{F} \in \mathfrak{M}_{H}(I, d) \mid \mathcal{F}_{\mid 2 C} \cong \mathcal{E}_{\mid 2 C}\right\}$, where $\mathcal{E}$ is a sheaf (on $X$ ) locally free along $C$.

It is clear that $\Lambda_{1}^{C}$ is closed and the others are locally closed in $\mathfrak{M}_{H}(I, d)$. Following [GL lemma 6.6], $\Lambda_{\mathcal{E}}^{C}$ is a subscheme of $\mathfrak{M}_{H}(I, d) .{ }^{3}$ Let $\mathcal{F} \in \Lambda_{\mathcal{E}}^{C}$. The Zariski tangent space of $\Lambda_{\mathcal{E}}^{C}$ at $\mathcal{F}$ is isomorphic to the kernel of the restriction homomorphism $\operatorname{Ext}_{X}^{1}(\mathcal{F}, \mathcal{F})^{0} \rightarrow \operatorname{Ext}_{2 C}^{1}\left(\mathcal{F}_{\mid 2 C}, \mathcal{F}_{\mid 2 C}\right)^{0}$.

Lemma 1.4. Let $\mathcal{E}$ be any locally free sheaf on $2 C$. Then away from $\Lambda_{1}^{C}$ the subscheme $\Lambda_{\mathcal{E}}^{C}$ is smooth and it meets $\mathcal{S}_{1} \mathfrak{M}_{H}(I, d)$ transversally.

Proof. This follows from a standard deformation argument. We omit the details here.

Next we estimate the codimension of the sets $\Lambda_{1}^{C}$ and $\Lambda_{2}^{C}$.

Lemma 1.5. There is an integer $N$ depending on $(X, I, \mathcal{C})$ such that whenever $d \geq N$ and $H \in \mathcal{C}$, then $\operatorname{codim}\left(\Lambda_{2}^{C}, \mathfrak{M}_{H}(I, d)\right) \geq 3 g+3$ and

$$
\operatorname{codim}\left(\Lambda_{1}^{C}, \mathfrak{M}_{H}(I, d)\right)>-3 \chi\left(\mathcal{O}_{2 C}\right)+3 g+14 .
$$

\footnotetext{
${ }^{3}$ By this we mean $\Lambda_{\mathcal{E}}^{C}$ is an open subset of a closed subscheme of $\mathfrak{M}_{H}(I, d)$.
} 
Proof. The proof given by [Do, $\mathrm{Fr}, \mathrm{Zu}$ ] can be adopted to cover the estimate of $\operatorname{codim}\left(\Lambda_{1}^{C}, \mathfrak{M}_{H}(I, d)\right)$. Now we show that by choosing $N$ large,

$$
\operatorname{codim}\left(\Lambda_{2}^{C}, \mathfrak{M}_{H}(I, d)\right) \geq 3 g+3 .
$$

Let $\mathcal{E} \in \Lambda_{2}^{C}$ be any sheaf. Then since $C^{2}>0$,

$$
\operatorname{dim} \operatorname{Ext}^{0}\left(\mathcal{E}_{\mid 2 C}, \mathcal{E}_{\mid 2 C}\right)^{0} \leq 2 \operatorname{dim} \operatorname{Ext}^{0}\left(\mathcal{E}_{\mid C}, \mathcal{E}_{\mid C}\right)^{0} .
$$

Thus $\Lambda_{2}^{C}$ is contained in the set $\Lambda^{\prime}$ of $\mathcal{E} \in \mathfrak{M}_{H}(I, d)$ such that $\mathcal{E}_{\mid C}$ is locally free and $\operatorname{Ext}^{0}\left(\mathcal{E}_{\mid C}, \mathcal{E}_{\mid C}\right)^{0}>2 g+6$. Thus it suffices to show that $\operatorname{codim}\left(\Lambda^{\prime}\right)>$ $3 g+3$. Let $\mathcal{E} \in \Lambda^{\prime}$. Since $\operatorname{dim} \operatorname{End}\left(\mathcal{E}_{\mid C}\right)^{0}>2 g+6, \mathcal{E}_{\mid C}$ is unstable. Let $\mathcal{L}_{1}$ be the destabilizing subsheaf of $\mathcal{E}_{\mid C}$ and $\mathcal{L}_{2}=\mathcal{E}_{\mid C} / \mathcal{L}_{1}$. Then since

$$
\operatorname{dim} \operatorname{End}\left(\mathcal{E}_{\mid C}\right)^{0} \leq 2+h^{0}\left(\mathcal{L}_{2}^{\vee} \otimes \mathcal{L}_{1}\right) \leq 2+\operatorname{deg}\left(\mathcal{L}_{2}^{\vee} \otimes \mathcal{L}_{1}\right),
$$

$\operatorname{deg} \mathcal{L}_{1} \geq \operatorname{deg} \mathcal{L}_{2}+2 g+4$, and then $\mathcal{E}_{\mid C} \cong \mathcal{L}_{1} \oplus \mathcal{L}_{2}$. Since $\mathcal{L}_{1} \otimes \mathcal{L}_{2} \cong \mathcal{O}_{C}\left(I_{\mid C}\right)$, the set $\left\{\mathcal{E}_{\mid C} \mid \mathcal{E} \in \Lambda^{\prime}\right\}$ is isomorphic to a subset of $\operatorname{Pic}(C)$. Next, for stable $\mathcal{E} \in \Lambda^{\prime}-\Lambda_{1}^{C}$, the tangent space of the set $\left\{\mathcal{E}^{\prime} \in \Lambda^{\prime} \mid \mathcal{E}_{\mid C}^{\prime} \cong \mathcal{E}_{\mid C}\right\}$ at $\mathcal{E}$ is the kernel of $\operatorname{Ext}_{X}^{1}(\mathcal{E}, \mathcal{E})^{0} \rightarrow \operatorname{Ext}_{C}^{1}\left(\mathcal{E}_{\mid C}, \mathcal{E}_{\mid C}\right)^{0}$ which has dimension no more than

$$
\operatorname{dim} \operatorname{Ext}_{X}^{1}(\mathcal{E}, \mathcal{E})^{0}-\operatorname{dim} \operatorname{Ext}_{C}^{1}\left(\mathcal{E}_{\mid C}, \mathcal{E}_{\mid C}\right)^{0}<\operatorname{dim} \mathfrak{M}_{H}(I, d)-(5 g+3),
$$

using the Riemann-Roch and $\operatorname{dim} \operatorname{Ext}_{C}^{0}\left(\mathcal{E}_{\mid C}, \mathcal{E}_{\mid C}\right)^{0}>2 g+6$. Hence

$$
\operatorname{codim}\left(\Lambda^{\prime}-\Lambda_{1}^{C}\right) \geq(5 g+3)-\operatorname{dim} \operatorname{Pic}(C)>4 g+3 .
$$

Finally, since $\operatorname{codim}\left(\Lambda_{1}^{C}\right) \geq 3 g+3$, we have $\operatorname{codim}\left(\Lambda_{2}^{C}\right)>3 g+3$ as desired. This proves the lemma.

In studying $\mathfrak{M}_{H}(I, d)$, we often need to use the local tautological family. Let $w \in \mathfrak{M}_{H}(I, d)$ be any closed point. A local tautological family of $\mathfrak{M}_{H}(I, d)$ at $w$ is an analytic (or étale) neighborhood $\varphi: U \rightarrow \mathfrak{M}_{H}(I, d)$ of $w$ and a family of sheaves $\mathcal{E}_{U}$ on $X \times U$ flat over $U$ such that for any $u \in U$, the restriction sheaf $\mathcal{E}_{U \mid X \times\{u\}}$ is represented by the $\varphi(u) \in \mathfrak{M}_{H}(I, d)$. By expressing $\mathfrak{M}_{H}(I, d)$ as G.I.T. quotient of the Grothendieck's quotient scheme and applying the étale slicing theorem, we have

Lemma 1.6. Any point $w \in \mathfrak{M}_{H}(I, d)$ admits a local tautological family.

Now we discuss how to construct the morphism (0.8). We fix an $N$ given by lemma 1.3. For any $d \geq N$, let $H \in \mathcal{C}$ be $\left(H_{0}, I, d\right)$-suitable. 
In [Li1], the author constructed a line bundle $\mathcal{L}_{C}$ over $\overline{\mathfrak{M}}_{H}(I, d)$ and the associated morphism $\Psi_{C}: \overline{\mathfrak{M}}_{H}(I, d) \longrightarrow \mathbf{P}^{R}$. The fiber of $\Phi_{C}$ containing an $\mathcal{F} \in \mathfrak{M}_{H}(I, d)$ is described by the set $S(\mathcal{F})$, which is

$$
\left\{\begin{array}{l|l}
\mathcal{E} \in \mathfrak{M}_{H}(I, d) \mid \begin{array}{l}
\mathcal{F}^{\vee} \cong \mathcal{E}^{\vee} \text { and } \\
\ell\left(\left(\mathcal{F}^{\vee \vee} / \mathcal{F}\right)_{x}\right) \cong \ell\left(\left(\mathcal{E}^{\vee \vee} / \mathcal{E}\right)_{x}\right) \text { for each } x \in X
\end{array}
\end{array}\right\}
$$

We summarize the property of this morphism as follows:

Lemma 1.7. Let $N$ be given in lemma 1.3 and let $d \geq N, H \in \mathcal{C}$ be $\left(H_{0}, I, d\right)$-suitable. Then there is a line bundle $\mathcal{L}_{C}$ on $\overline{\mathfrak{M}}_{H}(I, d)$ of which the following holds:

(1) For some large $m>0, H^{0}\left(\overline{\mathfrak{M}}_{H}(I, d), \mathcal{L}_{C}^{\otimes m}\right)$ is base point free. We now let $\Psi_{C}: \overline{\mathfrak{M}}_{H}(I, d) \rightarrow \mathbf{P}^{R}$ be the induced morphism;

(2) For any $\mathcal{E} \in \mathfrak{M}_{H}(I, d), \Psi_{C}^{-1}\left(\Psi_{C}(\mathcal{E})\right) \cap \mathfrak{M}_{H}(I, d)$ is identical to $S(\mathcal{E})$;

(3) There is a codimension $3 g-2$ linear subspace $V \subset \mathbf{P}^{R}$ such that $\Psi_{C}^{-1}(V)$ is exactly the set

$$
\operatorname{cl}(\mathcal{Y})=\left\{\mathcal{E} \in \overline{\mathfrak{M}}_{H}(I, d) \mid \mathcal{E}_{\mid C} \text { is either non-locally free or unstable }\right\} .
$$

Proof. Following [Li1], away from the set $W \subset \overline{\mathfrak{M}}_{H}(I, d)$, where $W$ consists of strictly $H$-semistable but $H_{0}-\mu$-unstable sheaves, one can construct a line bundle $\mathcal{L}_{C}$ satisfying (1) and (2). Namely, $H^{0}\left(\overline{\mathfrak{M}}_{H}(I, d)-W, \mathcal{L}_{C}^{\otimes m}\right)$ is base point free. (See Proposition 1.7 and 2.5 in [Li1] for the proof of (1) and Theorem 4 in [Li1] for the proof of (2).) Since $H$ is $\left(H_{0}, I, d\right)$-suitable, $W$ is empty. This proves (1) and (2). Now we prove (3). Let $\mathfrak{M}(C)$ be the moduli space of semistable vector bundles over $C$ of determinent $I_{\mid C}$ and let $\rho: \overline{\mathfrak{M}}_{H}(I, d)--\rightarrow \mathfrak{M}(C)$ be the rational map sending $\mathcal{E}$ to $\mathcal{E}_{\mid C}$ when it is semistable. Then there is an ample line bundle $L_{C}$ on $\mathfrak{M}(C)$ such that $\rho^{*}\left(L_{C}\right) \cong \mathcal{L}_{C}$ over where $\rho$ is defined. Further, for any $s \in H^{0}\left(\mathfrak{M}(C), L_{C}^{\otimes m}\right)$, $\rho^{*}(s)$ extends to $\overline{\mathfrak{M}}_{H}(I, d)$ and the vanishing locus of this extension consists of all $\mathcal{E}$ that satisfy one of the following conditions: (1) $\mathcal{E}_{\mid C}$ is non-locally free; (2) $\mathcal{E}_{\mid C}$ is locally free but unstable or (3) $\mathcal{E}_{\mid C}$ lies in $s^{-1}(0)$. (See [Li1, Proposition 2.5] or the proof of [Li2, Lemma 4.12] for proofs.) 'Thus, if we choose $3 g-2$ sections of $L_{C}^{\otimes m}$ with no common vanishing locus, then the extensions of their pull backs on $\overline{\mathfrak{M}}_{H}(I, d)$ will define a codimension $3 g-2$ linear subspace $V \subset \mathbf{P}^{R}$ that has the desired property. 
Note that in the proof, we only used the fact that all sheaves in $\overline{\mathfrak{M}}_{H}(I, d)$ are $H_{0}-\mu$-semistable. The extra requirement that $H$ be $(I, d)$-generic will be useful later because of the following lemma.

Lemma 1.8. Let $N$ be as before and let $d \geq N, H \in \mathcal{C}$ be $(I, d)$-generic. Then the subset $\mathcal{S} \overline{\mathfrak{M}}_{H}(I, d) \subset \overline{\mathfrak{M}}_{H}(I, d)$ is Cartier.

Proof. This lemma is proved in [Li3, Lemma 2.7]. Note that when $d$ is large and $H$ is $(I, d)$-generic, any semistable but not $\mu$-stable sheaf is non-locally free.

A recent result of O'Grady shows that there are no complete subsets of $\mathfrak{M}_{H}(I, d)^{0}$ of small codimension. As an application, we have the following existence Lemma:

Lemma 1.9. Let $C \subset X$ be as before and $H \in \mathcal{C}$ be any polarization. Assume $\mathcal{V}_{0}$ is a rank 2 locally free sheaf on $2 C$ such that $\operatorname{dim} \operatorname{Hom}_{2 C}\left(\mathcal{V}_{0}, \mathcal{V}_{0}\right) \leq$ $4 g+12$. Then there is an $N$ such that whenever $d \geq N, \mathcal{S}_{1} \mathfrak{M}_{H}(I, d)$ intersects each irreducible component of $\Lambda_{\mathcal{V}_{0}}^{C}-\Lambda_{1}^{C}$, assuming it is non-empty.

Proof. We first prove the lemma in case $H=H_{0}$. We let $\Lambda_{\mathcal{V}_{0}}^{C}\left(H_{0}, d\right)$ be the subset $\Lambda_{\mathcal{V}_{0}}^{C} \subset \mathfrak{M}_{H_{0}}(I, d)$ defined in (1.5) and let $R$ be an irreducible component of $\Lambda_{\mathcal{V}_{0}}^{C}\left(H_{0}, d\right)-\Lambda_{1}^{C}$. Note that the codimension of $R$ at a general $\mathcal{E} \in R$ is at most

$$
\operatorname{dim} \operatorname{Ext}_{2 C}^{1}\left(\mathcal{V}_{0}, \mathcal{V}_{0}\right)^{0}=-3 \chi\left(\mathcal{O}_{2 C}\right)+4 g+12
$$

By the main theorem proved in [OG2], for large $d$ the closure $\operatorname{cl}(R)$ of $R$ in $\overline{\mathfrak{M}}_{H_{0}}(I, d)$ intersects $\mathcal{S} \overline{\mathfrak{M}}_{H_{0}}(I, d)$. However, since the dimension of $\overline{\mathfrak{M}}_{H_{0}}(I, d)-\mathfrak{M}_{H_{0}}(I, d)$ is much less than the dimension of $R$ for large $d$ by Lemma 1.3, $\operatorname{cl}(R)$ intersects $\mathcal{S M}_{H_{0}}(I, d)$. It remains to show that $R \cap$ $\mathcal{S}_{1} \mathfrak{M}_{H_{0}}(I, d) \neq \emptyset$. Indeed, by combining the deformation argument in the proof of Theorem 6.2 of [GL] and the estimate of $\operatorname{codim}\left(\Lambda_{1}^{C}\right)$, we know that $c l(R)-R$ can not be a codimension one subset of $\operatorname{cl}(R)$. Therefore, $R \cap$ $\mathcal{S} \mathfrak{M}_{H_{0}}(I, d) \neq \emptyset$ because $\mathcal{S} \mathfrak{M}_{H_{0}}(I, d)$ is a divisor. By the same deformation argument again, one concludes that $R$ contains sheaves $\mathcal{E} \in \mathcal{S} \mathfrak{M}_{H_{0}}(I, d)$ that has length $\ell\left(\mathcal{E}^{\vee \vee} / \mathcal{E}\right)=1$. This shows that $R \cap \mathcal{S}_{1} \mathfrak{M}_{H_{0}}(I, d) \neq \emptyset$.

Now we prove the Lemma for arbitrary $H \in \mathcal{C}$. By (6) of Lemma 1.3, there are $B^{\prime} \subset \Lambda_{\mathcal{V}_{0}}^{C}(d, H)$ and $B_{0}^{\prime} \subset \Lambda_{\mathcal{V}_{0}}^{C}\left(d, H_{0}\right)$ such that

$$
\Lambda_{\mathcal{V}_{0}}^{C}(d, H)-B^{\prime} \cong \Lambda_{\mathcal{V}_{0}}^{C}\left(d, H_{0}\right)-B_{0}^{\prime}
$$


and that the codimension of $B^{\prime}$ in $\Lambda_{\mathcal{V}_{0}}^{C}(d, H)$ and that of $B_{0}^{\prime}$ in $\Lambda_{\mathcal{V}_{0}}^{C}\left(d, H_{0}\right)$ are at least 2. Therefore since $\Lambda_{\mathcal{V}_{0}}^{C}\left(d, H_{0}\right) \cap \mathcal{S}_{1} \mathfrak{M}_{H_{0}}(I, d)$ is non-empty and is a divisor of $\Lambda_{\mathcal{V}_{0}}^{C}\left(d, H_{0}\right)$,

$$
\left(\Lambda_{\mathcal{V}_{0}}^{C}\left(d, H_{0}\right)-B_{0}^{\prime}\right) \cap \mathcal{S}_{1} \mathfrak{M}_{H_{0}}(I, d) \neq \emptyset .
$$

This shows that $\Lambda_{\mathcal{V}_{0}}^{C}(d, H) \cap \mathcal{S}_{1} \mathfrak{M}_{H}(I, d)$ is non-empty. This proves the Lemma.

We close this part of discussion with a lemma concerning the normal bundle of $\mathcal{S}_{1} \mathfrak{M}_{H}(I, d) \subset \mathfrak{M}_{H}(I, d)$.

Lemma 1.10. Let $\mathcal{F} \in \mathcal{S}_{1} \mathcal{M}_{d}$ be any sheaf and let $S(\mathcal{F}) \subset \mathcal{S}_{1} \mathcal{M}_{d}$ be defined in (1.6). Then there is a normal slice $U$ of $\mathcal{S}_{1} \mathcal{M}_{d} \subset \mathcal{M}_{d}$ along $S(\mathcal{F})$ such that $U-S(\mathcal{F})$ has trivial first and second homology groups (with rational coefficients).

Here by a normal slice of $\mathcal{S}_{1} \mathcal{M}_{d} \subset \mathcal{M}_{d}$ we mean an analytic submanifold $U$ that intersect $\mathcal{S}_{1} \mathcal{M}_{d}$ transversally along $\mathcal{S}(\mathcal{F})$.

Proof. Let $S=S(\mathcal{F})$. Clearly, $S \cong \mathbf{P}^{1}$ since $\mathcal{E} \in S$ are constructed as kernels of $\mathcal{F}^{\vee \vee} \rightarrow \mathcal{O}_{x}$, where $x=\operatorname{supp}\left(\mathcal{F}^{\vee \vee} / \mathcal{F}\right)$. From Lemma 1.7, we know that the morphism $\Psi_{C}$ contracts $\mathcal{S}_{1} \mathcal{M}_{d}$ to $X \times \mathcal{M}_{d-1}^{0}$ whose fibers are exactly $S(\mathcal{E})$ for $\mathcal{E} \in \mathcal{S}_{1} \mathcal{M}_{d}$. Therefore, if we choose a smocth analytic surface $N$ containing $S\left(=\mathcal{S}(\mathcal{F})\right.$ for some $\left.\mathcal{F} \in \mathcal{S}_{1} \mathcal{M}_{d}\right)$ that rneets $\mathcal{S}_{1} \mathcal{M}$ transversally along $S \subset \mathcal{S}_{1} \mathcal{M}_{d}$, then the restriction of $\Psi_{C}$ to $N$ contracts $S \subset N$. Therefore, the normal bundle of $S \subset N$ is $\mathcal{O}_{S}(-l)$, the line bundle of degree $-l<0$, and a tubular neighborhood $U$ of $S \subset N$, which is a normal slice of $\mathcal{S}_{1} \mathcal{M}_{d} \subset \mathcal{M}_{d}$ along $S$, satisfies $H_{1}(U-S)=H_{2}(U-S)=0$. This proves the lemma.

Remark 1.11. A straightforward calculation shows that $S \subset N$ is indeed a $(-2)$-curve. Since we do not need this information in this paper, we will leave its proof to the readers.

In the remainder of this section, we will consider issues concerning homology of singular spaces. One type of technical results that we need in the future says that for certain closed subset $\Lambda \subset \overline{\mathfrak{M}}_{H}(I, d)$,

$$
H_{i}\left(\overline{\mathfrak{M}}_{H}(I, d)-\Lambda, \mathcal{S} \overline{\mathfrak{M}}_{H}(I, d)-\Lambda\right) \longrightarrow H_{i}\left(\overline{\mathfrak{M}}_{H}(I, d), \mathcal{S} \overline{\mathfrak{M}}_{H}(I, d)\right)
$$

is an isomorphism. This type of results are certainly known to the experts. Due to the lack of reference, we shall provide proofs of them. 
Lemma 1.12. Let $Z \subset \mathbf{P}^{n+r}$ be any irreducible quasi-projective variety of pure dimension $n$ and $\Lambda, V \subset Z$ two closed algebraic subsets $(V$ may be empty).

(1) Assume $V$ is Cartier, $\Lambda \subset Z$ has codimension at least $k$ and $Z-V$ is locally defined by at most $r+l$ equations, then

$$
H_{i}(Z-\Lambda, V-\Lambda) \longrightarrow H_{i}(Z, V)
$$

is an isomorphism for $i<(k-l)-1$ and is surjective for $i=(k-l)-1$.

(2) Assume $Z \subset \mathbf{P}^{n+r}$ is locally defined by at most $r+l$ equations and $T \subset Z$ is the locus where $Z$ is not locally irreducible. Then $\operatorname{dim} T \geq$ $n-l-2$.

Proof. Let $\mathcal{S}$ be a Whitney stratification of $Z$ by algebraic subsets so that $V$ and $\Lambda$ are union of strata. Let $S_{1}, \cdots, S_{h}$ be strata of $\Lambda$ with nondecreasing dimensions and let $\Lambda_{i}=\cup_{j \leq i} S_{j}$. Then the lemma follows if the homomorphism

$$
H_{i}\left(Z-\Lambda_{j}, V-\Lambda_{j}\right) \longrightarrow H_{i}\left(Z-\Lambda_{j-1}, V-\Lambda_{j-1}\right)
$$

has the stated property for all $j$. Because of this observation, we only need to prove the lemma under the assumption that $\Lambda$ is already a stratum in $\mathcal{S}$. Since $\Lambda \subset Z$ is a stratum and $\Lambda \subset Z$ is closed, there is a compact (in the ordinary topology) $\Lambda_{0} \subset \Lambda$ such that $\left((Z-\Lambda) \cup \Lambda_{0},(V-\Lambda) \cup \Lambda_{0}\right)$ has the same homology group as $(Z, V)$. Hence it suffices to show that

$$
H_{i}(Z-\Lambda, V-\Lambda) \longrightarrow H_{i}\left((Z-\Lambda) \cup \Lambda_{0},(V-\Lambda) \cup \Lambda_{0}\right)
$$

has the desired property. Let $p_{0} \in \Lambda_{0}$ and let $N_{p_{0}}$ be a normal slice of $\Lambda \subset Z$ at $p_{0}$. We claim that the pair

$$
\left(\partial B_{\varepsilon}\left(p_{0}\right) \cap N_{p_{0}}, \partial B_{\varepsilon}\left(p_{0}\right) \cap N_{p_{0}} \cap V\right)
$$

is homologically $(k-l-2)$-connected for $0<\varepsilon \ll 1$. Indeed, the case when $p_{0} \in V$ follows directly from theorem 2 and the remark preceding it on page 156 of $[\mathrm{GM}]$. When $p_{0} \notin V$, we let $\left(p_{0}, N_{p_{0}}\right) \subset\left(0, \mathbb{C}^{R}\right)$ be an embedding and let $h_{1}, \cdots, h_{t}, t=R-\operatorname{dim} N_{p_{0}}+r$, be the defining equations of $N_{p_{0}}$. Then the claim follows from applying the same theorem in [GM] to map $\pi: \mathbb{C}^{R} \rightarrow \mathbb{C}^{R+t}, \pi(z)=(z, h .(z))$, and the linear subspace $C^{R} \times\{0\} \subset \mathbb{C}^{R+t}$. 
Now it is easy to prove (1) of the Lemma. By Thom's first isotopy lemma [GM], we can find a set $U \subset(Z-\Lambda) \cup \Lambda_{0}$ containing; $\Lambda_{0}$ with a projection $\pi: U \rightarrow \Lambda_{0}$ such that the restriction of $\pi$ to $\Lambda_{0}$ is identity and that $\pi: U \rightarrow \Lambda_{0}$ is an $N_{p_{0}}$ bundle over $\Lambda_{0}$. (i.e. $U$ is a union of normal slices of $\Lambda \subset Z$ at $p \in \Lambda_{0}$, each homeomorphic to $N_{p_{0}}$.) Because (1.7) is $(k-l-2)$ connected, $\left(U-\Lambda_{0},\left(U-\Lambda_{0}\right) \cap V\right)$ is $(k-l-2)$-connected as well. Finally, we apply the Mayer-Vietoris sequence to pairs $(Z-\Lambda, V-\Lambda)$ and $(U, U \cap V)$. Because $(U, U \cap V)$ has trivial homology groups and $\left(U-\Lambda_{0},\left(U--\Lambda_{0}\right) \cap V\right)$ is $(k-l-2)$-connected, (1.6) is an isomorphism for $i<k-l-1$ and surjective for $i=k-l-1$. This proves the first part of the Lemma.

For the second part, we let $p \in T$ be a general point and $N_{p}$ be a normal slice of $T \subset Z$ at $p$. Since $T$ is the locus of points where $Z$ is not locally irreducible and $p \in T$ general, $\partial B_{\epsilon}(p) \cap N_{p}$ is not connected for $0<\epsilon \ll 1$. However by the proof of $(1), H_{0}\left(\partial B_{\epsilon}(p) \cap N_{p}\right)=\mathbb{Q}$ if $\operatorname{codim}(T, Z)-l-2>0$. Thus we have $\operatorname{codim}(T, Z) \leq l+2$. This proves the Lemma.

For $T \subset Z$ that does not have the property of the previous Lemma, we still can choose representative of any homological class that intersects $T$ in the "least" possible way.

Lemma 1.13. Assume $\Lambda, V \subset Z$ as in the previous Lemma. Assume $\operatorname{dim} \Lambda \leq n-1, \operatorname{dim} \Lambda \cap V \leq n-2$ and $V \subset Z$ is Cartier, then for $i=1$ or 2 , any class in $H_{i}(Z, V)$ can be represented by a cycle $\Sigma$ such, that $\Sigma \cap \Lambda$ has real dimension at most $i-1$ and $\Sigma \cap \Lambda \cap V$ has real dimension at most $i-2$. If $Z-V$ is locally irreducible near $\Lambda$, then we can choose $\Sigma$ so that $\Sigma \cap \Lambda$ and $\Sigma \cap \Lambda \cap V$ have real dimension at most $i-2$. Furihermore, if $\operatorname{dim} \Lambda \cap V \leq n-3$ and $Z-V$ is locally irreducible, then we can choose $\Sigma$ such that $\Sigma \cap \Lambda \cap V$ has real dimension at most $i-3$.

Proof. We prove the case where $i=2, V=\emptyset$ and $Z$ is locally irreducible. The other case is similar. Note that the only case we need in this paper is when $i=1$ and 2 . Let $\mathcal{S}$ be a Whitney stratification of $Z$ by subvarieties such that $\Lambda$ is a union of strata. Let $S_{1}, \cdots, S_{r}$ be strata in $\Lambda$ of nor.-decreasing dimension. We pick a 2-cycle $\Sigma$ representing a class in $H_{2}(Z)$. We say $\Sigma$ meets $\Lambda$ generically near $A \subset \Lambda$ if there is an analytic neighborhood $O_{A}$ of the closure $\bar{A}$ of $A \subset Z$ such that $\Sigma \cap \Lambda \cap O_{A}$ is discrete. We claim that if $\Sigma$ meets $\Lambda$ generically near $S_{1} \cup \cdots \cup S_{r}$, then we can find a new representative $\tilde{\Sigma}$ so that $\tilde{\Sigma}$ meets $\Lambda$ generically near $S_{1} \cup \cdots \cup S_{r+1}$. Note that then by induction on $r$, we get a desired cycle $\Sigma^{\prime}$ that intersects $\Lambda$ at discrete points. 
We now prove the claim. Let $S=S_{r+1}$. Without loss of generality we can assume $\Sigma \cap S$ is a piecewise smooth subset. Let $W=\Sigma \cap S$. Since the closure of $S$ is contained in the union of $S_{1}, \cdots, S_{r}, W$ is compact. Thus we can find a compact neighborhood $N$ of $W$ such that $N$ is a fiber bundle over $W_{0}, W \subset W_{0} \subset S$, with projection $\pi: N \rightarrow W_{0}$, such that whose fibers are homeomorphic to $N_{p} \cap B_{\epsilon}(p)$, where $N_{p}$ is a normal slice of $S \subset Z$ at $p \in S$ and $0<\epsilon \ll 1$. Since $Z$ is locally irreducible along $S, \partial \pi^{-1}(p t)$ is non-empty and connected. Hence by a standard obstruction argument, we can find a cycle $\tilde{\Sigma}$ such that $\tilde{\Sigma}|Z-\stackrel{\circ}{N}=\Sigma| Z-\stackrel{\circ}{N}$ and $\tilde{\Sigma} \cap S$ is discrete, where ${ }_{N}^{\circ}$ is the interior of $N$. Not only that, by perturbing $\tilde{\Sigma}$ in a small neighborhood $U$ of $\tilde{\Sigma} \cap S$ we can assume $\tilde{\Sigma} \cap U \cap \Delta=\tilde{\Sigma} \cap S$. Thus $\tilde{\Sigma}$ meets $S_{1}, \cdots, S_{r+1}$ generically. This proves the claim and then the first part of the Lemma. The furthermore part can be proved using the argument after (1.7) and that of [GM].

Lemma 1.14. Let $S \in \mathcal{S}$ be any stratum and let $q \in \bar{S} \subset Z$ be any point. Then if $\Sigma \subset Z$ is a closed cycle contained in $\cup_{i \in A} S_{i}$, where $A$ is a subset of $\mathcal{S}$, and $\Sigma \cap S$ is discrete, then we can find a new representative $\Sigma^{\prime} \subset Z$ of $[\Sigma] \in H_{i}(Z)$ such that $\Sigma^{\prime} \subset\left(\cup_{j \in A} S_{j}-S\right) \cup\{q\}$.

Proof. Let $U$ be a cone neighborhood of $q \in Z$ that respects the stratification $\mathcal{S}$. Let $p \in \Sigma \cap S$. We choose a differentiable path $\rho:[0,1] \rightarrow S$ connecting $p$ with a $p_{1} \in U$ and let $N_{[0,1]}$ be a continuous family of normal slices of $S \subset Z$ along $\rho([0,1])$. By shrinking $N_{[0,1]}$ if necessary, we can assume $N_{[0,1]}$ is homeomorphic to $N_{p} \times[0,1]$, say by $\Psi: N_{p} \times[0,1] \rightarrow N_{[0,1]}$. Next, by perturbing $\Sigma$ near $p$, we can assume $\Sigma \cap B_{\varepsilon}(p) \subset N_{p}$ for $0<\varepsilon \ll 1$. We fix a sufficiently small $\varepsilon>0$ so that $\Psi\left(\left(B_{\varepsilon}(p) \cap N_{p}\right) \times\{1\}\right) \subset U$. Now let $A_{1}=\Sigma-B_{\varepsilon}(p), A_{2}=\Psi(\Gamma \times[0,1])$, where $\Gamma=\partial\left(\Sigma \cap B_{\varepsilon}(p)\right)$ and $A_{3}$ is the cone over $\Psi(\Gamma \times\{1\})$ in $U$. Then $\Sigma^{\prime}=A_{1} \cup A_{2} \cup A_{3}$ is a representative of $[\Sigma]$ with $\#\left(\Sigma^{\prime} \cap S\right)<\#(S)$. By performing the above perturbation at each $p \in \Sigma \cap S$, we will get a desired representative of $[\Sigma] \in H_{*}(Z)$.

\section{Unstable sheaves over curves.}

The goal of this section is to investigate the local irreducibility of the space of $\mu$-unstable sheaves over a smooth curve. This will be needed in studying the singularity of the set $\mathcal{Y} \subset \mathfrak{M}_{H}(I, d)$ mentioned in the introduction. 
We first fix the convention we will use throughout this section. Let $C$ be a smooth curve of genus $g \geq 3$ and $\overline{\mathcal{E}}$ a rank $2 \mu$-unstable sheaf on $C$. Since we will be interested in sheaves with torsions, we will generalize the notion of $\mu$-stability to arbitrary sheaves. A sheaf $\mathcal{F}$ of $\operatorname{rank} \mathcal{F}>0$ on $C$ is $\mu$-stable (resp. $\mu$-semistable) if for any locally free quotient sheaf $\mathcal{F} \rightarrow \mathcal{L}$ with $\operatorname{rank} \mathcal{L}<\operatorname{rank} \mathcal{F}$, we have $\mu(\mathcal{F})<\mu(\mathcal{L})$ (resp. $\leq$ ). Note that there are sheaves with torsions that are $\mu$-stable. As usual, $\mu$-stable and $\mu$-semistable are open conditions. Let $\left(A, \mathcal{F}_{A} ; 0, \overline{\mathcal{E}}\right)$ be a versal deformation space of $\overline{\mathcal{E}}$ of fixed determinant of even degree. (We will recall the definition of versal deformation space in Definition 2.3.) Here, $A$ is a scheme containing 0 and $\mathcal{F}_{A}$ a sheaf on $C \times A$ restricting to $\overline{\mathcal{E}}$ along $C \times\{0\}$. We define

$$
A_{0}=\left\{w \in A \mid \mathcal{F}_{w} \text { is } \mu \text {-unstable }\right\} .
$$

$A_{0}$ is closed in $A$. Since $\overline{\mathcal{E}}$ is $\mu$-unstable, $0 \in A_{0}$. As usual, we say a variety $Z$ is locally irreducible at $z \in Z$ if for $0<\varepsilon \ll 1$ the smooth locus of the $\varepsilon$-neighborhood $B_{\varepsilon}(z) \cap Z$ is connected. In this section, we shall prove the following technical results concerning $A_{0} \subset A$.

Proposition 2.1. With the notation as above, then the subvariety $A_{0} \subset A$ has pure complex codimension $g+1$ at $0 \in A_{0}$.

Proposition 2.2. Assume $\overline{\mathcal{E}}$ is locally free, then the subvariety $A_{0}$ is locally irreducible at $0 \in A_{0}$.

We first recall the notion of algebraic versal deformation space of $\overline{\mathcal{E}}$.

Definition 2.3. Let $Z$ be a projective scheme and let $\overline{\mathcal{E}}$ be a rank two sheaf over $Z$ with $\operatorname{det} \overline{\mathcal{E}} \cong M$. An algebraic versal deformation space of $\overline{\mathcal{E}}$ (resp. of fixed determinant) is a collection $\left(A, \mathcal{F}_{A} ; 0, \overline{\mathcal{E}}\right)$, where $0 \in A$ is a quasi-projective scheme and $\mathcal{F}_{A}$ is an algebraic family of sheaves on $Z \times A$ flat over $A$ (resp. with $\operatorname{det} \mathcal{F}_{A} \cong p_{Z}^{*} M, p_{Z}: Z \times A \rightarrow Z$ ) such that

(1) the restriction of $\mathcal{F}_{A}$ to $Z \times\{0\}$, say $\mathcal{F}_{0}$, is isomorphic to $\overline{\mathcal{E}}$, and that the Kodaira-Spencer map $T_{0} A \rightarrow \operatorname{Ext}^{1}\left(\mathcal{F}_{0}, \mathcal{F}_{0}\right)^{0}$ induced by the family $\mathcal{F}_{A}$ is an isomorphism;

(2) For any pair of affine varieties $S_{0} \subset S$ coupled with a sheaf $\mathcal{E}_{S}$ on $Z \times S$ flat over $S$ such that $S_{0} \subset S$ is closed, $\operatorname{det} \mathcal{E}_{S} \cong ! p_{Z}^{*} M$ and $\mathcal{E}_{S \mid Z \times S_{0}} \cong q_{Z}^{*} \overline{\mathcal{E}}_{\mid Z \times S_{0}}$, where $q_{Z}: Z \times S_{0} \rightarrow Z$, there is an analytic neighborhood $U$ of $S_{0} \subset S$ and an analytic map $\eta:\left(U, S_{0}\right) \rightarrow(A, 0)$ so that the restriction of $\mathcal{E}_{S}$ to $Z \times U$ is isomorphic to $\left(1_{Z} \times \eta\right)^{*} \mathcal{F}_{A}$, extending the given isomorphism $\mathcal{F}_{0} \cong \overline{\mathcal{E}}$ and $\mathcal{E}_{S \mid Z \times S_{0}} \cong q_{Z}^{*} \overline{\mathcal{E}}$. 
The situation in which we need the versal deformation space is either when $Z=C$, or when $Z=2 C$ and $\overline{\mathcal{E}}$ is locally free $(2 C$ is a subscheme of $X$, where $C \subset X$ is a smooth curve in $\left.\left|n_{0} H\right|\right)$. The existence of versal deformation spaces in these settings is known for long time (see $[\mathrm{Ar}]$ ). Here we outline the proof for $Z=C$ since we need some properties of this deformation space that can not be found in literatures.

We now sketch the construction of versal deformation space of $\overline{\mathcal{E}}$ of fixed determinant on $C$. By choosing a sufficiently ample line bundle $H$ on $C$, we can express $\overline{\mathcal{E}}$ as a quotient sheaf of $\mathcal{R}=\oplus^{N} \mathcal{O}\left(H^{-1}\right)$ with $N=h^{0}(\overline{\mathcal{E}} \otimes$ $\mathcal{O}(H))$. Let $\mathfrak{Q}\left(\operatorname{resp} \mathfrak{Q}_{0}\right)$ be the Grothendieck's Quot-scheme parameterizing all rank 2 quotient sheaves $\mathcal{F}$ of $\mathcal{R}$ (resp. with $\operatorname{det} \mathcal{F} \cong M$ ). We fix a point $0 \in \mathfrak{Q}$ so that $\mathcal{F}_{0} \cong \overline{\mathcal{E}}$ and the associated quotient sheaf $\mathcal{R} \stackrel{\sigma_{0}}{\longrightarrow} \mathcal{F}_{0}$ induces isomorphism $\mathbb{C}^{N} \cong H^{0}\left(\mathcal{F}_{0} \otimes \mathcal{O}(H)\right)$. Because $C$ is a smooth curve and $H$ is sufficiently ample, $\mathfrak{Q}$ is smooth at 0 [Ma, p594]. Further, the morphism $\mathfrak{Q} \rightarrow \operatorname{Pic}(C)$ that sends $\mathcal{R} \rightarrow \mathcal{F}$ to $\operatorname{det} \mathcal{F}$ is a submersion. Hence $\mathfrak{Q}_{0}$, which is the preimage scheme of $M \in \operatorname{Pic}(C)$, is smooth as well. Also, the tangent space of $\mathfrak{Q}_{0}$ at 0 belongs to the exact sequence

$$
0 \rightarrow \operatorname{Hom}\left(\mathcal{F}_{0}, \mathcal{F}_{0}\right) \rightarrow \operatorname{Hom}\left(\mathcal{R}, \mathcal{F}_{0}\right) \stackrel{j}{\longrightarrow} T_{0} \mathfrak{Q}_{0} \rightarrow \operatorname{Ext}^{1}\left(\mathcal{F}_{0}, \mathcal{F}_{0}\right)^{0} \rightarrow 0
$$

Now we let $A \subset \mathfrak{Q}_{0}$ be an affine smooth subvariety containing 0 so that the induced homomorphism

$$
T_{0} A \hookrightarrow T_{0} \mathfrak{Q}_{0} \longrightarrow \operatorname{Ext}^{1}\left(\mathcal{F}_{0}, \mathcal{F}_{0}\right)^{0}
$$

is an isomorphism. Let $\mathcal{F}_{A}$ be the restriction to $C \times A$ of the universal quotient family. Then the data $\left(A, \mathcal{F}_{A} ; 0, \mathcal{F}_{0}\right)$ satisfies 1$)$ of the definition 2.3 .

Now, we show that they also satisfy 2) of the definition 2.3. Let $S_{0} \subset$ $S$ and $\mathcal{E}_{S}$ be given in (2). Since $\mathcal{E}_{S \mid Z \times S_{0}}$ is isomorphic to the pull-back of $\mathcal{F}_{0}(=\overline{\mathcal{E}})$, we can express $\mathcal{E}_{S \mid Z \times S_{0}}$ as the quotient sheaf of $q_{Z}^{*} \mathcal{R}$ using $\mathcal{R} \stackrel{\sigma_{0}}{\longrightarrow} \mathcal{F}_{0}$. Since $H$ is sufficiently ample, that $S_{0}$ is affine and closed in $S$, we can extend $q_{Z}^{*} \mathcal{R} \rightarrow \mathcal{E}_{S \mid Z \times S_{0}}$ to a neighborhood of $S_{0} \subset S$. Then by shrinking $S$ if necessary (still containing $S_{0}$ ), there is a morphism $g: S \rightarrow \mathfrak{Q}_{0}$ with $g\left(S_{0}\right)=0$ so that $\mathcal{E}_{S}$ is isomorphic to the pullback of the universal quotient family. Next we will find an analytic neighborhood $U$ of $0 \in \mathfrak{Q}_{0}$ and an analytic map $\pi_{+}: U \rightarrow A$ so that $\pi_{+} \circ g$ is the desired map $\eta$. Note that $\mathfrak{Q}_{0}$ is a $G$-scheme with $G=G L(N, \mathbb{C})=\operatorname{Hom}(\mathcal{R}, \mathcal{R})$. Let $G_{0}$ be the stabilizer of $0 \in \mathfrak{Q}_{0}$ and let $N$ be a normal slice of $G_{0} \subset G$ at $1 \in G_{0}$. Then the orbit $N \cdot 0$ is smooth at 0 and its tangent space at 0 is the image $j\left(\operatorname{Hom}\left(\mathcal{R}, \mathcal{F}_{0}\right)\right) \subset T_{0} \mathfrak{Q}_{0}$. Thus $N \cdot 0$ and $A$ meets transversally at 0 . In 
particular, there is an analytic neighborhood $V_{-}$of $1 \in N$ and an analytic neighborhood $V_{+}$of $0 \in A$ so that $V_{-} \times V_{+} \rightarrow \mathfrak{Q}_{0},\left(u_{-}, u_{+}\right) \mapsto u_{-} \cdot u_{+}$, is one-to-one. Let $\mathcal{U}$ be the image of $V_{-} \times V_{+} \rightarrow \mathfrak{Q}_{0}$ and let

$$
\pi_{ \pm}: \mathcal{U} \longrightarrow V_{ \pm}
$$

be the induced projections. Then for any analytic variety $B$ and any analytic $\operatorname{map} \xi: B \rightarrow \mathcal{U} \subset \mathfrak{Q}_{0}, \xi(z)=\pi_{-} \circ \xi(z) \cdot\left(\pi_{+} \circ \xi(z)\right)$. Hence the sheaf $\mathcal{E}_{B}$ in the pullback quotient family $h_{B}: p_{C}^{*} \mathcal{R} \rightarrow \mathcal{E}_{B}$ via $1_{C} \times \xi$ and the sheaf $\mathcal{E}_{B}^{\prime}$ in the pullback quotient family $h_{B}^{\prime}: p_{C}^{*} \mathcal{R} \rightarrow \mathcal{E}_{B}^{\prime}$ via $1_{C} \times\left(\pi_{+} \circ \xi\right)$ are isomorphic (analytically). Back to $g: S \rightarrow \mathfrak{Q}_{0}$, we let $U=g^{-1}(\mathcal{U})$ and $\eta=\pi_{+} \circ g: U \rightarrow A$. The previous reasoning shows that $(\eta, U)$ sa.tisfying (2) of the Definition.

Remark 2.4. For $w \in V_{+} \subset A$, because $T_{w} \mathfrak{Q}_{0} \rightarrow \operatorname{Ext}^{1}\left(\mathcal{F}_{w}, \mathcal{J}_{w}\right)^{0}$ is surjective and $T_{w}\left(V_{-} \cdot w\right) \rightarrow \operatorname{Ext}^{1}\left(\mathcal{F}_{w}, \mathcal{F}_{w}\right)^{0}$ is trivial, $T_{w} A \rightarrow \operatorname{Ext}^{1}\left(\mathcal{F}_{w}, \mathcal{F}_{w}\right)^{0}$ must be surjective.

We now prove a technical lemma concerning components of germ of $0 \in A_{0}$. In the following, for any point $w$ in an algebraic set $W$, we will use $\operatorname{germ}(W, w)$ to denote the $\varepsilon$-ball $B_{\varepsilon}(w)$ of $w \in W$, where $0<\varepsilon \ll 1$, under some Riemannian metric.

Lemma 2.5. Let $\mathcal{E}_{S}$, where $s_{0} \in S$ is a smooth curve, be a family of $\mu$ unstable sheaves satisfying $\mathcal{E}_{s_{0}} \cong \overline{\mathcal{E}}$. Then if $s_{0} \in U \subset S$ and $\eta_{1}$ and $\eta_{2}$ are two analytic maps from $\left(U, s_{0}\right)$ to $(A, 0)$ given by (2) of the definition 2.3 based on the family $\mathcal{E}_{S}$, then the images $\eta_{1}\left(\operatorname{germ}\left(U, s_{0}\right)\right)$ and $\eta_{2}\left(\operatorname{germ}\left(S, s_{0}\right)\right)$ are contained in the same irreducible component of $\operatorname{germ}\left(A_{0}, 0\right)$.

Proof. We continue to use the notation developed in the argument after Definition 2.3. Let $\mathcal{E}_{U}=\mathcal{E}_{S \mid C \times U}$. Since $A \subset \mathfrak{Q}_{0}$, the map $\eta_{i}: U \rightarrow A$ is given by quotient sheaf homomorphism $f_{i}: p_{C}^{*} \mathcal{R} \rightarrow \mathcal{E}_{U}$, which is determined by the induced isomorphism $\tilde{f}_{i}: \oplus^{N} \mathcal{O}_{C \times U} \rightarrow p_{U *}\left(\mathcal{E}_{U} \otimes p_{C}^{*} \mathcal{O}(H)\right.$ ) (at; least near $\left.0 \in \mathfrak{Q}_{0}\right)$. Let $\tilde{g}=\tilde{f}_{2}^{-1} \circ \tilde{f}_{1} \in \operatorname{Hom}\left(\mathcal{O}_{C \times U}^{\oplus N}, \mathcal{O}_{C \times U}^{\oplus N}\right)$. $\tilde{g}$ induces a homomorphism $g: p_{C}^{*} \mathcal{R} \rightarrow p_{C}^{*} \mathcal{R}$ such that $f_{1}=f_{2} \circ g$. Since $\eta_{1}\left(s_{0}\right)=\eta_{2}\left(s_{0}\right)$ as quotient sheaves, $\tilde{g}\left(s_{0}\right)=c \cdot i d$ for some constant $c$. Next, let $T$ be a connected analytic neighborhood of $1 \in G L(N)$ and let $\Psi: T \times U \rightarrow \mathfrak{Q}_{0}$ be the map defined by $\Psi(h, s)=h \cdot \eta(s)$. By shrinking $T$ and $U$ if necessary, we can assume the composition of $\Psi$ with the local projection $\pi_{+}$of (2.2) is well-defined. Then

$\eta_{i}\left(\operatorname{germ}\left(U, s_{0}\right)\right) \subset \pi_{+} \circ \Psi\left(\operatorname{germ}\left(T \times U,\left(1, s_{0}\right)\right)\right) \subset \operatorname{germ}\left(A_{0}, 0\right) \quad$ for $i=1,2$. 
Because $T$ is irreducible, $\eta_{1}\left(\operatorname{germ}\left(U, s_{0}\right)\right)$ and $\eta_{2}\left(\operatorname{germ}\left(U, s_{0}\right)\right)$ must be contained in the same irreducible component of germ $\left(A_{0}, 0\right)$. This completes the proof of the lemma.

We now turn our attention to the proof of the Proposition 2.1 and 2.2. For simplicity we will assume $\operatorname{deg} \overline{\mathcal{E}}$ is even, since these are the only cases needed in this paper. The proofs of the odd degree cases are parallel. From now on, we assume $\operatorname{deg} \overline{\mathcal{E}}$ is even. Then by tensoring $\overline{\mathcal{E}}$ with $\mathcal{M}^{-1}$, where $\mathcal{M}^{\otimes 2} \cong \operatorname{det} \overline{\mathcal{E}}$, we can assume without loss of generality that $\operatorname{det} \overline{\mathcal{E}} \cong \mathcal{O}_{C}$. Let $\mathcal{U}_{1}$ be the set of all $\mu$-unstable sheaves of determinant $\mathcal{O}_{C}$ whose destabilizing (locally free) quotient sheaves have degree -1 and $\mathcal{U}_{1}^{0}$ be the subset of $\mathcal{U}_{1}$ consisting of sheaves having no traceless automorphisms. Note that sheaves in $\mathcal{U}_{1}^{0}$ are necessarily locally free.

We first show that unstable sheaves can be deformed to sheaves in $\mathcal{U}_{1}^{0}$. We will consider the case of locally free sheaves in Lemma 2.6 and non-locally free case in Lemma 2.7.

Lemma 2.6. Let $\mathcal{E}$ be a rank 2 locally free $\mu$-unstable sheaf with $\operatorname{det} \mathcal{E} \cong$ $\mathcal{O}_{C}$, then there is a deformation of $\mathcal{E}$ so that whose generic members are in $\mathcal{U}_{1}^{0}$.

Proof. Since $\mathcal{E}$ is $\mu$-unstable and locally free, we can find a degree 1 invertible sheaf $\mathcal{L}$ and possibly a 0 -subscheme (divisor) $z \subset C$ such that

$$
0 \longrightarrow \mathcal{L} \longrightarrow \mathcal{E} \longrightarrow \mathcal{L}^{-1}(-z) \oplus \mathcal{O}_{z} \longrightarrow 0
$$

is exact. We choose a smooth affine curve $s_{0} \in S$ and an invertible sheaf $\mathcal{L}_{S}$ on $C \times S$ such that $\mathcal{L}_{S \mid C \times\left\{s_{0}\right\}} \cong \mathcal{L}$ and that for general $s \in S$, $H^{0}\left(\mathcal{L}_{S \mid C \times\{s\}}^{\otimes 2}\right)=0$ (note $g(C) \geq 3$ ). We now consider the sheaves $\mathcal{L}_{S}$ and $\mathcal{L}_{S}^{-1} \otimes \mathcal{I}$ on $C \times S$, where $\mathcal{I}$ is the ideal sheaf of $z \times\left\{s_{0}\right\} \in C \times S$. Clearly, both sheaves are flat over $S$ and the restriction of $\mathcal{L}_{S}^{-1} \otimes \mathcal{I}$ to $C \times\left\{s_{0}\right\}$ is $\mathcal{L}^{-1}(-z) \oplus \mathcal{O}_{z}$. We consider the $\mathcal{O}_{S}$-module $\operatorname{Ext}_{C \times S}^{1}\left(\mathcal{L}_{S}^{-1} \otimes \mathcal{I}, \mathcal{L}_{S}\right)$ and the restriction homomorphism

$$
\operatorname{Ext}_{C \times S}^{1}\left(\mathcal{L}_{S}^{-1} \otimes \mathcal{I}, \mathcal{L}_{S}\right) \stackrel{\text { rest }}{\longrightarrow} \operatorname{Ext}_{C}^{1}\left(\mathcal{L}^{-1}(-z) \oplus \mathcal{O}_{z}, \mathcal{L}\right) .
$$

By hase change and cohomology theorem, (2.5) is surjective since all higher extension groups vanish. Therefore we can find a

$$
v \in \operatorname{Ext}_{C \times S}^{1}\left(\mathcal{L}_{S}^{-1} \otimes \mathcal{I}, \mathcal{L}_{S}\right)
$$


so that whose associated exact sequence

$$
0 \longrightarrow \mathcal{L}_{S} \longrightarrow \mathcal{E} \longrightarrow \mathcal{L}_{S}^{-1} \otimes \mathcal{I} \longrightarrow 0
$$

restricts to (2.4) along $C \times\left\{s_{0}\right\}$. Finally by choosing $v$ general, we can assume the restriction of the above exact sequence to general fibers $C \times\{s\}$ does not split. Therefore for general $s \in S H^{0}\left(\mathcal{E} n d^{0}\left(\mathcal{E}_{s}\right)\right)=0$. This proves Lemma 2.6.

Lemma 2.7. Let $\mathcal{E}$ be any rank two $\mu$-unstable sheaf with torsion having $\operatorname{det} \mathcal{E} \cong \mathcal{O}_{C}$, then there is

(1) a deformation of $\mathcal{E}$ so that whose general members are $\mu$-unstable and locally free;

(2) a deformation of $\mathcal{E}$ so that whose generic members $\mathcal{E}_{v}$ are in $\mathcal{U}_{1}$ and $\ell\left(\mathcal{E}_{v}^{t}\right)=1$, where $\mathcal{E}_{v}^{t}$ is the torsion part of $\mathcal{E}_{v}$.

Proof. Let $\mathcal{L}$ be the (locally free) destabilizing quotient sheaf of $\mathcal{E}$. Then there is another invertible sheaf $\mathcal{M}$ and a torsion sheaf $\mathcal{T}$ such that

$$
0 \longrightarrow \mathcal{M} \oplus \mathcal{T} \longrightarrow \mathcal{E} \longrightarrow \mathcal{L} \longrightarrow 0
$$

is exact. Obviously, we can deform $\mathcal{E}$ with fixed determinant so that the general members belong to $(2.6)$ with $\mathcal{M}$ (resp. $\mathcal{L}$ ) replaced by invertible sheaf $\mathcal{M}^{\prime}$ (resp. $\mathcal{L}^{\prime}$ ) of identical degree and $\mathcal{T}$ replaced by $\oplus_{i=1}^{r} \mathcal{O}_{x_{i}}$, where $x_{i} \in C$ are distinct. Since deformations of deformations are deformations, to prove this lemma it suffices to consider those $\mathcal{E}$ whose torsion $\mathcal{T}=\oplus_{i=1}^{r} \mathcal{O}_{x_{i}}$. We first prove (1). Let $z=\cup_{i=1}^{r} x_{i} \subset C$ be the 0 -subscheme. Then $\mathcal{M} \oplus \mathcal{T}$ is the restriction to $C \times\left\{s_{0}\right\} \subset C \times S$, where $s_{0} \in S$ is a smooth affine curve, of the sheaf $p_{C}^{*} \mathcal{L}^{-1} \otimes \mathcal{I}$. ( $\mathcal{I}$ is still the ideal sheaf of $z \times\left\{s_{0}\right\} \subset C \times S$.) Similar to the proof of Lemma 2.6, we can choose an element in $\operatorname{Ext}_{C \times S}^{1}\left(p_{C}^{*} \mathcal{L}, p_{C}^{*} \mathcal{L}^{-1} \otimes \mathcal{I}\right)$ whose associated exact sequence

$$
0 \longrightarrow p_{C}^{*} \mathcal{L}^{-1} \otimes \mathcal{I} \longrightarrow \mathcal{E}_{S} \longrightarrow p_{C}^{*} \mathcal{L} \longrightarrow 0
$$

restricts to (2.6) along $C \times\left\{s_{0}\right\}$. (This is possible again by the base change and cohomology theorem.) The general members of $\mathcal{E}_{S}$ satisfy the conclusion of (1). Next we construct deformations satisfying (2). Let $\mathcal{E}^{\prime}=\mathcal{E} / \mathcal{O}_{x_{1}}$. Then using the argument for (1) we can find a deformation of $\mathcal{E}^{\prime}$ with fixed determinant such that whose general members are locally free and have 
$\mathcal{L}$ as their destabilizing quotient sheaves. Let $\mathcal{E}_{v}$ be its general member. Note that $\operatorname{det} \mathcal{E}_{v} \cong \mathcal{O}_{C}\left(-x_{1}\right)$. Then following the argument in the proof of Lemma 2.6, we can deform $\mathcal{E}_{v}$ (determinant fixed) to sheaves having degree -1 destabilizing quotient sheaves, say $\mathcal{E}_{w}$. Therefore, $\mathcal{E}_{w} \oplus \mathcal{O}_{x_{1}}$ is a deformation of $\mathcal{E}$ whose general members belong to $\mathcal{U}_{1}$ and have length 1 torsions. This proves the second part of the Lemma.

With the material prepared, it is now easy to prove the Proposition 2.1.

Proof of proposition 2.1. Since every $\mu$-unstable sheaf $\mathcal{E}$ admits a deformation whose general member belongs to $\mathcal{U}_{1}^{0}, A_{0} \cap \mathcal{U}_{1}^{0}$ is dense in $A_{0}$. Thus to prove Proposition 2.1 it suffices to show that for $w \in A_{0} \cap \mathcal{U}_{1}^{0}$ close to 0 , $\operatorname{codim}\left(A_{0} \cap \mathcal{U}_{1}^{0}\right.$ at $\left.w\right)=g+1$. Let $\mathcal{F}_{A}$ be the family associated to the versal deformation space $A$ of $\overline{\mathcal{E}}$, let $w \in A_{0} \cap \mathcal{U}_{1}^{0}$ and let $\left(A^{\prime}, \mathcal{F}_{A^{\prime}} ; 0^{\prime}, \mathcal{F}_{w}\right)$ be the versal deformation space of $\mathcal{F}_{w}$. Then there is an analytic neighborhood $U$ of $w \in A$ and an analytic map $f:(U, w) \rightarrow\left(A^{\prime}, 0^{\prime}\right)$ such that $\left(1_{C} \times f\right)^{*} \mathcal{F}_{A^{\prime}}$ is (analytically) isomorphic to $\mathcal{F}_{A}$ restricting to $C \times U$. Note, $U \cap \mathcal{U}_{1}^{0}=f^{-1}\left(A^{\prime} \cap \mathcal{U}_{1}^{0}\right)$. Therefore Proposition 2.1 follows from (a) $\operatorname{codim}\left(A^{\prime} \cap \mathcal{U}_{1}^{0}, A^{\prime}\right)=g+1$ and (b) $f$ is a submersion at $w$. We first prove (b). Since both $A$ and $A^{\prime}$ are smooth, it suffices to show that the differential $d f: T_{w} A \rightarrow T_{0^{\prime}} A^{\prime}$ is surjective. But this follows from the remark 2.4 since $w \in A_{0}$ is close to 0 . For a), we first note that the obvious map $A^{\prime} \cap \mathcal{U}_{1}^{0} \rightarrow \mathcal{U}_{1}^{0}$ is one-to-one, at least near $0^{\prime} \in A^{\prime}$. Hence $\operatorname{dim}\left(A^{\prime} \cap \mathcal{U}_{1}^{0}\right)=\operatorname{dim} \mathcal{U}_{1}^{0}$. We claim $\operatorname{dim} \mathcal{U}_{1}^{0}=2 g-4$. Indeed, since sheaves in $\mathcal{U}_{1}^{0}$ belong to (non-splitting) exact sequences

$$
0 \longrightarrow \mathcal{L} \longrightarrow \mathcal{E} \longrightarrow \mathcal{L}^{-1} \longrightarrow 0
$$

with $\operatorname{deg} \mathcal{L}=1$ and $H^{0}\left(\mathcal{L}^{\otimes 2}\right)=0$, the correspondence between $\mathcal{E} \in \mathcal{U}_{1}^{0}$ and exact sequence (2.6) is one-to-one modulo automorphisms of $\mathcal{L}^{-1}$. Hence the dimension of $\mathcal{U}_{1}^{0}$ at $\mathcal{E}$ (in (2.6)) is

$$
\operatorname{dim} \operatorname{Pic}(C)+\operatorname{dim} \operatorname{Ext}^{1}\left(\mathcal{L}^{-1}, \mathcal{L}\right)-1
$$

which is $g+(g-4)$. On the other hand, since $\operatorname{Ext}^{0}\left(\mathcal{F}_{w}, \mathcal{F}_{w}\right)^{0}$ is trivial, $A^{\prime}$ is smooth at $0^{\prime}$ of dimension $\operatorname{dim} \operatorname{Ext}^{1}\left(\mathcal{F}_{w}, \mathcal{F}_{w}\right)^{0}$, which is $3 g-3$. Therefore $\operatorname{codim}\left(A^{\prime} \cap \mathcal{U}_{1}^{0}, A^{\prime}\right)=g+1$. This completes the proof of proposition 2.1.

We now turn our attention to the proof of proposition 2.2 .

Proof of proposition 2.2. Let $l$ be the integer so that $\overline{\mathcal{E}}$ has degree $-(1+l)$ destabilizing quotient sheaf. Let $B=\operatorname{germ}\left(A_{0}, 0\right)$. By Lemma 2.6, $B \cap \mathcal{U}_{1}$ 
is dense in $B$. Since sheaves in $\mathcal{U}_{1}$ all have degree -1 destabilizing quotient sheaves, we can define a morphism

$$
p: B \cap \mathcal{U}_{1} \longrightarrow \operatorname{Pic}^{-1}(C)
$$

that sends $\mathcal{F}$ to its destabilizing quotient sheaf. (Superscript -1 stands for the degrees of bundles). Clearly, for each irreducible component $R$ of $B$, $p\left(R \cap \mathcal{U}_{1}\right)$ is not contained in any subvariety in $\mathrm{Pic}^{-1}(C)$. Hence for any two irreducible components $R_{1}$ and $R_{2}$ of $B$, we can find a smooth (analytic) curve $s_{0} \in S$, a morphism $j:\left(s_{0}, S\right) \rightarrow \operatorname{Pic}^{-1}(C)$ and morphisms $r_{i}:\left(s_{0}, S\right) \rightarrow$ $\left(0, A_{0}\right)$, where $i=1,2$, such that $r_{i}\left(S-s_{0}\right) \subset \mathcal{U}_{1}^{0}$, germ $\left(0, r_{i}(S)\right) \subset R_{i}$ and that after restricting to $r_{i}^{-1}\left(R_{i} \cap \mathcal{U}_{1}\right)$ the following triangle are commutative:

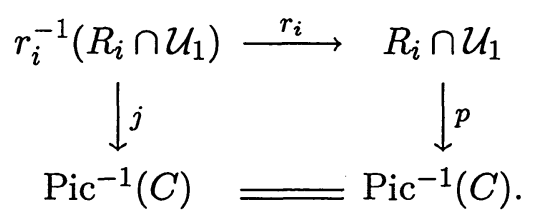

Let $\mathcal{L}$ be the invertible sheaf on $C \times S$ that is the pull-back of the Poincare bundle on $C \times \mathrm{Pic}^{-1}(C)$ via $1_{C} \times j$. We need the following technical result that will be proved shortly.

Sublemma. There is a 1-dimensional subscheme $D_{i} \subset C \times S$ flat over $S$ with $\operatorname{deg}\left(D_{i} \cap C \times\left\{s_{0}\right\}\right)=l$ and a locally free sheaf $\mathcal{G}_{i}$ on $C \times S$ such that $\mathcal{G}_{i}$ belongs to the exact sequence

$$
0 \longrightarrow \mathcal{L}^{-1}\left(D_{i}\right) \longrightarrow \mathcal{G}_{i} \longrightarrow \mathcal{L} \longrightarrow 0,
$$

and $\left(1_{C} \times r_{i}\right)^{*}\left(\mathcal{F}_{A}\right)$ and $\mathcal{G}_{i}$ are related by the exact sequence

$$
0 \longrightarrow\left(1_{C} \times r_{i}\right)^{*} \mathcal{F}_{A} \longrightarrow \mathcal{G}_{i} \longrightarrow \mathcal{O}_{D_{i}} \longrightarrow 0 .
$$

We continue our proof of the Proposition assuming this Sublemma. The strategy is to construct a family $\widetilde{\mathcal{F}}$ of $\mu$-unstable locally free sheaves on $C \times Z$, where $Z$ is smooth and irreducible, that has the following properties: (2.9) There are curves $\tilde{r}_{i}: S \rightarrow Z$ with $i=1,2$ such that the pull-backs $\left(1_{C} \times \tilde{r}_{i}\right)^{*} \widetilde{\mathcal{F}}$ are isomorphic to $\left(1_{C} \times r_{i}\right)^{*} \mathcal{F}_{A}$ respectively and (2.10) there is a connected $Z_{0} \subset Z$ containing $\tilde{r}_{1}\left(s_{0}\right)$ and $\tilde{r}_{2}\left(s_{0}\right)$ such that $\widetilde{\mathcal{F}}_{\mid C \times Z_{0}}$ is isomorphic to $p_{C}^{*} \overline{\mathcal{E}}$, where $p_{C}: C \times Z_{0} \rightarrow C$.

Once we have such a family, then we take an affine $T \subset Z$ such that $T_{0}=$ $T \cap Z_{0}$ is closed in $T$ and contains $\tilde{r}_{1}\left(s_{0}\right)$ and $\tilde{r}_{2}\left(s_{0}\right)$. Then $T_{0} \subset T$ with the family $\tilde{\mathcal{F}}_{\mid C \times T}$ satisfy the condition in (2) of Definition 2.3 . Therefore, we get 
a morphism $f: U \rightarrow A_{0}$ defined over an analytic neighborhood $U$ of $T_{0} \subset T$. By Lemma 2.5, $f\left(U \cap \tilde{r}_{i}(S)\right)$ and $r_{i}(S)$ are contained in the same irreducible component. However, since $T$ is irreducible and smooth and $T_{0}$ is connected, $B \cap f(U)$ is contained in an irreducible component of $B$, after shrinking $U$ if necessary. This is possible only if $r_{1}(S)$ and $r_{2}(S)$ are contained in the same irreducible component of $B$. Since we can choose $r_{1}$ and $r_{2}$ general, $R_{1}$ and $R_{2}$ must be identical. This will complete the proof of the Proposition.

We now construct the family $\widetilde{\mathcal{F}}$ on $C \times Z$ with the desired properties. Morally speaking, $Z$ will be a variety consisting of data $\left\{\mathcal{G} \rightarrow \mathcal{O}_{D}\right\}$, where $D$ is a degree $l$ effective divisor in $C, \mathcal{G}$ is an extension of $\mathcal{L}$ by $\mathcal{L}^{-1}(D)$ for some degree -1 invertible sheaf $\mathcal{L}$. Let $T$ be an affine open subset of Hilb $^{l}(C)$ containing $D_{1} \cap C \times\left\{s_{0}\right\}$ and $D_{2} \cap C \times\left\{s_{0}\right\}$, and let $D$ be the restriction to $C \times T$ of the universal divisor. We consider the sheaves $\pi_{C \times S}^{*} \mathcal{L}$ and $\pi_{C \times S}^{*} \mathcal{L}^{-1}(\tilde{D})$ on $C \times S \times T$ and the relative extension sheaf

$$
\mathcal{E} x t_{C \times S \times T / S \times T}^{1}\left(\pi_{C \times S}^{*} \mathcal{L}, \pi_{C \times S}^{*} \mathcal{L}^{-1}(\tilde{D})\right),
$$

where $\pi_{C \times S}$ and $\pi_{C \times T}$ are projections from $C \times S \times T$ to its factors and $\tilde{D}=\pi_{C \times T}^{-1}(D)$. Since $(2.11)$ is coherent, we can represent it as a quotient sheaf of a locally free sheaf $\mathcal{V}$ with the quotient homomorphism $q$. Let $V$ be the total space of the vector bundle associated to $\mathcal{V}, \pi: V \rightarrow S \times T$ the projection and $\varphi \in H^{0}\left(\pi^{*} \mathcal{V}\right)$ the tautological section (i.e. for any $v \in V$ over $x \in S \times T, \varphi_{\mid v}=v \in \mathcal{V}_{\mid x}$ ). We denote $p_{C \times S}$ (resp. $p_{C \times T}$ ) be the obvious projection from $C \times V$ to $C \times S$ (resp. $C \times T$ ). Then there is a rank 2 locally free sheaf $\widetilde{\mathcal{G}}$ on $C \times V$ belonging to the exact sequence

$$
0 \longrightarrow p_{C \times S}^{*} \mathcal{L}^{-1}\left(p_{C \times T}^{-1}(D)\right) \longrightarrow \widetilde{\mathcal{G}} \longrightarrow p_{C \times S}^{*} \mathcal{L} \longrightarrow 0
$$

defined by the extension class that is the image of the (tautological) section $\varphi$ under

$$
\begin{aligned}
\pi^{*} \mathcal{V} \stackrel{\pi^{*} q}{\longrightarrow} \pi^{*} \mathcal{E} x t_{C \times S \times T / S \times T}^{1}\left(\pi_{C \times S}^{*} \mathcal{L}, \pi_{C \times S}^{*} \mathcal{L}^{-1}\left(\pi_{C \times T}^{-1}(D)\right)\right) \\
\longrightarrow \mathcal{E} x t_{C \times V / V}^{1}\left(p_{C \times S}^{*} \mathcal{L}, p_{C \times S}^{*} \mathcal{L}^{-1}\left(p_{C \times T}^{-1}(D)\right)\right)
\end{aligned}
$$

(Note that $V$ is affine since $T$ and $S$ are both affine.) We next construct the family $\widetilde{\mathcal{F}}$. We consider the sheaf

$$
\mathcal{W}=\pi_{V *} \mathcal{H}\left(\mathbb{I}_{C \times V}\left(\widetilde{\mathcal{G}}, \mathcal{O}_{p_{C \times T}^{-1}(D)}\right), \quad \pi_{V}: C \times V \longrightarrow V .\right.
$$

$\mathcal{W}$ is locally free since $\widetilde{\mathcal{G}}$ is locally free and $p_{C \times T}^{-1}(D)$ is flat over $V$. Let $\mathbf{W}$ be the vector bundle associated to $\mathcal{W}$ and let $Z^{\prime}$ be the associated projective 
bundle $\mathbf{P}(\mathbf{W})$ with projection $\tilde{\pi}: Z^{\prime} \rightarrow V$. (Here we use the convention that $\mathbf{P}\left(\mathbb{C}^{n}\right)=\mathbb{C}^{n}-\{0\} / \mathbb{C}^{*}$.) Let

$$
\mathcal{H}:\left(1_{C} \times \tilde{\pi}\right)^{*} \widetilde{\mathcal{G}} \longrightarrow\left(1_{C} \times \tilde{\pi}\right)^{*} \mathcal{O}_{p_{C \times T}^{-1}(D)} \otimes \mathcal{O}_{Z^{\prime}}(1)
$$

be the tautological homomorphism. We let $Z \subset Z^{\prime}$ be the open subset consisting of those $z \in Z^{\prime}$ such that the restriction $\mathcal{H}_{\mid C \times\{z\}}$ are surjective, and let $\widetilde{\mathcal{F}}$ be the restriction to $C \times Z$ of the kernel $\operatorname{ker}\left\{\mathcal{H}_{C \times Z^{\prime}}\right\}$. We claim that $(Z, \tilde{\mathcal{F}})$ is the data we want.

We first show that $\widetilde{\mathcal{F}}$ satisfies condition (2.9). Let $f_{1}^{\prime}, f_{2}^{\prime}: S \rightarrow \operatorname{Hilb}^{l}(C)$ be morphisms induced by divisors $D_{1}$ and $D_{2}$ respectively. After shrinking $S$ if necessary we can assume $f_{i}^{\prime}$ factor through $T$. We denote by $f_{i}: S \rightarrow T$ the induced morphism. Thus $\left(1_{C} \times f_{i}\right)^{-1}(D)=D_{i}$. Then (2.7) assciciates to a section $\alpha_{i} \in \operatorname{Ext}_{C \times S}^{1}\left(\mathcal{L}, \mathcal{L}^{-1}\left(D_{i}\right)\right)$ that induces a curve $g_{i}: S \rightarrow V$ commuting with the projection $V \rightarrow S$. By the construction of $\tilde{\mathcal{G}},\left(1_{C} \times g_{i}\right)^{*} \widetilde{\mathcal{G}} \cong \mathcal{G}_{i}$. Further the quotient homomorphism

$$
\left(1_{C} \times g_{i}\right)^{*} \widetilde{\mathcal{G}} \equiv \mathcal{G}_{i} \longrightarrow \mathcal{O}_{D_{i}} \equiv \mathcal{O}_{\left(1_{C} \times f_{i}\right)^{-1}(D)}
$$

induced by (2.8) will provide us morphism $\tilde{r}_{i}: S \rightarrow Z$ that satisfies $\left(1_{C} \times\right.$ $\left.\tilde{r}_{i}\right)^{*} \widetilde{\mathcal{F}} \cong\left(1_{C} \times r_{i}\right)^{*} \mathcal{F}_{A}$, as desired.

We now show that the family satisfies (2.10). Let $Z_{s_{0}}$ be the fiber of $Z$ over $s_{0} \in S$ and let $Z_{0}$ be the subset of $z \in Z_{s_{0}}$ such that $\widetilde{\mathcal{F}}_{\mid C \times\{z\}} \cong \overline{\mathcal{E}}$. We claim that $Z_{0}$ is connected. Let $z_{1}, z_{2} \in Z_{0}$ be any two points. Let $t\left(z_{i}\right) \in T$ be the image in $T$ of $z_{i}$ under the obvious projection and $D_{t\left(z_{i}\right)} \subset C$ the divisor corresponding to $t\left(z_{i}\right) \in T$. By construction, $\overline{\mathcal{E}}=\tilde{\mathcal{F}}_{\mid C \times\left\{z_{i}\right\}}$ belongs to the exact sequence

$$
0 \longrightarrow \overline{\mathcal{E}} \longrightarrow \mathcal{G}_{i} \longrightarrow \mathcal{O}_{D_{t\left(z_{i}\right)}} \longrightarrow 0
$$

and $\mathcal{G}_{i}$ belongs to the exact sequence

$$
0 \longrightarrow \mathcal{L}_{0}^{-1}\left(D_{t\left(z_{i}\right)}\right) \longrightarrow \mathcal{G}_{i} \longrightarrow \mathcal{L}_{0} \longrightarrow 0
$$

where $\mathcal{L}_{0}=\mathcal{L}_{\mid C \times\left\{s_{0}\right\}}$. Also the cokernel of $\mathcal{G}_{i}\left(-D_{t\left(z_{i}\right)}\right) \rightarrow \overline{\mathcal{E}}$ induced by (2.12) is $\mathcal{O}_{D_{t\left(z_{i}\right)}}$, since $\mathcal{G}_{i}$ has degree $l$ and has degree -1 destabilizing quotient sheaf, and $\overline{\mathcal{E}}$ has degree 0 and has degree $-(1+l)$ destabilizing quotient sheaf. Hence

$$
0 \longrightarrow \mathcal{G}_{i}\left(-D_{t\left(z_{i}\right)}\right) \longrightarrow \overline{\mathcal{E}} \stackrel{\alpha_{i}}{\longrightarrow} \mathcal{O}_{D_{t\left(z_{i}\right)}} \longrightarrow 0
$$

is exact. Obviously, one can recover (2.12) from (2.13). Now we prove the connectivity of $Z_{0}$. Since $T$ is irreducible, there is an irreducible and affine 
curve $R \subset T$ containing $z_{1}$ and $z_{2}$. Let $D_{R}$ be the restriction of the universal divisor $D$ to $C \times R$. Then since $R$ is affine, we can find a homomorphism

$$
\alpha_{R}: \overline{\mathcal{E}} \otimes_{\mathcal{O}_{C}} \mathcal{O}_{C \times R} \longrightarrow \mathcal{O}_{D_{R}}
$$

such that its restriction to $C \times\left\{z_{i}\right\}$ is $\alpha_{i}$ in (2.13). Let $\mathcal{G}_{R}\left(-D_{R}\right)$ be the kernel of $\alpha_{R}$ and let $R_{0} \subset R$ be the open subset of $w \in R$ such that $\mathcal{G}_{R \mid C \times\{w\}}$ have degree -1 destabilizing quotient sheaves. Then if we let $\mathcal{L}_{R_{0}}$ be the sheaf on $C \times R_{0}$ that is the pull-back of $\mathcal{L}_{0}$ via the projection $C \times R_{0} \rightarrow C$, then $\mathcal{G}_{R_{0}}$ belongs to the exact sequence

$$
0 \longrightarrow \mathcal{L}_{R_{0}}^{-1}\left(D_{R_{0}}\right) \longrightarrow \mathcal{G}_{R_{0}} \longrightarrow \mathcal{L}_{R_{0}} \longrightarrow 0
$$

where $D_{R_{0}}=\left(D_{R} \cap C \times R_{0}\right)$. From our construction of $Z,(2.15)$ provides us a curve $R_{0} \rightarrow V_{s_{0}}$, where $V_{s_{0}}$ is the fiber of $V$ over $s_{0}$, and then (2.14) provides us a curve $R_{0} \rightarrow Z_{s_{0}}$ that contains $z_{1}$ and $z_{2}$. Since $R_{0}$ is irreducible and $z_{1}, z_{2} \in Z_{0}$ is arbitrary, $Z_{0}$ is irreducible. This completes the proof of Proposition 2.2.

We now provide the proof of the Sublemma.

Proof of the Sublemma. We let $i$ be either 1 or 2 and let $\mathcal{E}_{S}=\left(1_{C} \times\right.$ $\left.r_{i}\right)^{*} \mathcal{F}_{A}$. Because $r_{i}\left(S-s_{0}\right) \subset A_{0} \cap \mathcal{U}_{1}^{0}$ and because destabilizing subsheaves of unstable (locally free) sheaf is unique, there is an invertible sheaf $\mathcal{L}_{S}$ on $C \times S$ so that for $s \in S-s_{0}, \mathcal{L}_{s}$ is the destabilizing subsheaf of $\mathcal{E}_{s}$. Hence there is a homomorphism $\mathcal{L}_{S} \rightarrow \mathcal{E}_{S}$ that induces an exact sequence

$$
0 \longrightarrow \mathcal{L}_{S} \longrightarrow \mathcal{E}_{S} \stackrel{\beta}{\longrightarrow} \mathcal{L}_{S}^{-1} \otimes \mathcal{I}_{\Sigma} \longrightarrow 0,
$$

where $\mathcal{I}_{\Sigma}$ is the ideal sheaf of a zero-scheme $\Sigma \subset C \times S$ supported on $C \times\left\{s_{0}\right\}$. We claim that there is an effective divisor $D_{S} \subset C \times S$ containing $\Sigma$ flat over $S$ such that $D_{S}$ has degree $l$ along fibers of $C \times S \rightarrow S$, i.e. $D_{S} \cap\left(C \times\left\{s_{0}\right\}\right)$ has degree $l$. Indeed, since $\mathcal{E}_{s}$ is locally free at each $z \in \operatorname{supp}(\Sigma), \mathcal{I}_{\Sigma, z}$ is generated by two sections, say $f_{1}^{z}, f_{2}^{z} \in \mathcal{O}_{C \times S, z}$. Without loss of generality, we can assume

(2.17) $\left.f_{1}^{z}\right|_{C \times\left\{s_{0}\right\}}$ generates the locally free part of $\left.\mathcal{I}_{\Sigma}\right|_{C \times\left\{s_{0}\right\}}$ at $z$.

Then the union of $\left\{f_{1}^{z}=0\right\} \subset C \times S$ for $z \in \operatorname{supp}(\Sigma)$ form a divisor $D_{S}$ flat over $S$ near the fiber $C \times\left\{s_{0}\right\}$. By shrinking $S$ if necessary, we can assume $D_{S}$ is flat over $S$. We now check that it has degree $l$ along the fiber $C \times\left\{s_{0}\right\}$. Because of (2.17), by restricting (2.16) to $C \times\left\{s_{0}\right\}$ we get

$$
0 \longrightarrow \mathcal{L}_{s_{0}} \longrightarrow \mathcal{E}_{s_{0}} \stackrel{\beta_{s_{0}}}{\longrightarrow} \mathcal{L}_{s_{0}}^{-1}\left(-D_{s_{0}}\right) \oplus \tau \longrightarrow 0,
$$


where $D_{s_{0}}=D_{S} \cap\left(C \times\left\{s_{0}\right\}\right)$ and $\tau$ is a torsion sheaf. From this we see $\operatorname{deg} D_{s_{0}}=l$ since $\mathcal{E}_{s_{0}}=\overline{\mathcal{E}}$ has destabilizing quotient sheaf of degree $-(1+l)$ and $\operatorname{deg} \mathcal{L}_{s_{0}}=1$.

Now by taking the preimage of $\mathcal{L}_{S}^{-1}\left(-D_{S}\right) \subset \mathcal{L}_{S}^{-1} \otimes \mathcal{I}_{\Sigma}$ under $\beta$ in (2.16), we get a locally free sheaf $\mathcal{F}_{S}$ that belongs to the exact sequence

$$
0 \longrightarrow \mathcal{L}_{S} \longrightarrow \mathcal{F}_{S} \longrightarrow \mathcal{L}_{S}^{-1}\left(-D_{S}\right) \longrightarrow 0
$$

and the exact sequence

$$
0 \longrightarrow \mathcal{F}_{S} \longrightarrow \mathcal{E}_{S} \longrightarrow \mathcal{T} \longrightarrow 0
$$

where $\mathcal{T}=\mathcal{L}_{S}^{-1} \otimes \mathcal{I}_{\Sigma} / \mathcal{L}_{S}^{-1}\left(-D_{S}\right)$. Since $\mathcal{I}_{\Sigma}$ is generated by $f_{1}^{z}$ and $f_{2}^{z}$ and $\mathcal{L}_{S}^{-1}\left(-D_{S}\right)$ is generated by $f_{1}^{z}$ at $z \in \operatorname{supp}(\Sigma), \mathcal{T}=\mathcal{O}_{D_{S}}$. Therefore, we can recover $\mathcal{E}_{S}$ by

$$
0 \longrightarrow \mathcal{E}_{S} \longrightarrow \mathcal{F}_{S}\left(D_{S}\right) \stackrel{\sigma_{S}}{\longrightarrow} \mathcal{O}_{D_{S}} \longrightarrow 0 .
$$

Thus $\mathcal{G}_{i}=\mathcal{F}_{S}\left(D_{S}\right)$ with exact sequence (2.18) and (2.19) is the data required in the Sublemma. This finishes the proof of the Sublemma.

\section{Deformation of locally free sheaves.}

In this section, we will construct the homology mentioned in the introduction that will lead us to the proof of the vanishing of (0.9).

We fix $\left(H_{0}, I\right)$, a $C \in\left|n_{0} H_{0}\right|$ as in (1.1), a precompact neighborhood $\mathcal{C} \Subset \mathrm{NS}_{\mathbb{Q}}^{+}$of $H_{0} \in \mathrm{NS}_{\mathbb{Q}}^{+}$and the integer $N$ given in Lemma 1.3 and 1.5. We let $\mathcal{W} \subset \mathfrak{M}_{H}(I, d)$ be the subset consisting of $\mathcal{E}$ such that $\mathcal{E}_{\mid C}$ is locally free and unstable. In this section, we will prove

Proposition 3.1. Let $d \geq N$ and let $H \in \mathcal{C}$ be $\left(H_{0}, I, d\right)$-suitable, then we have

$$
H_{i}\left(\mathcal{W}, \mathcal{W} \cap \mathcal{S} \mathfrak{M}_{H}(I, d)\right)=0, \quad i \leq 2 .
$$

To prove this proposition, we need to show that every homology cycle $\left(\Sigma^{i}, \partial \Sigma^{i}\right) \rightarrow\left(\mathcal{W}, \mathcal{W} \cap \mathcal{S} \mathfrak{M}_{H}(I, d)\right)$ is homologous to zero. We will construct such homology directly by using the deformation of sheaves that will be constructed shortly.

We will construct such deformation of sheaves following [La, DG1]. The idea is as follows: Let $\mathcal{E}$ be any rank 2 locally free sheaf on $X$ of determinant $I$ such that its restriction to $C$ has destabilizing quotient sheaf $\mathcal{L}$ of degree 
$\frac{1}{2} I \cdot C-1$. (Note $I \cdot C$ is even.) By abuse of notation, we will view $\mathcal{L}$ as a sheaf of $\mathcal{O}_{X}$-modules by the inclusion $C \subset X$. Let $\mathcal{F}$ be the kernel of $\mathcal{E} \rightarrow \mathcal{L}$. Then $\mathcal{E}$ and $\mathcal{F}$ are related by the exact sequence

$$
0 \longrightarrow \mathcal{F} \longrightarrow \mathcal{E} \longrightarrow \mathcal{L} \longrightarrow 0 .
$$

Since the composite $\mathcal{E}(-C) \rightarrow \mathcal{E} \rightarrow \mathcal{L}$ is trivial, we have $\mathcal{E}(-C) \subset \mathcal{F}$, which induces the exact sequence

$$
0 \longrightarrow \mathcal{E}(-C) \longrightarrow \mathcal{F} \longrightarrow \mathcal{L}^{-1} \otimes \mathcal{O}_{C}\left(I_{\mid C}\right) \longrightarrow 0 .
$$

By replacing $\mathcal{L}^{-1} \otimes \mathcal{O}_{C}\left(I_{\mid C}\right)$ with a degree $\frac{1}{2} I \cdot C+1$ invertible sheaf of $\mathcal{O}_{C}$-modules $\mathcal{L}^{\prime}$, we obtain $\mathcal{E}^{\prime}$ based on the exact sequence

$$
0 \longrightarrow \mathcal{E}^{\prime}(-C) \longrightarrow \mathcal{F} \longrightarrow \mathcal{L}^{\prime} \longrightarrow 0
$$

that are deformations of $\mathcal{E}$. Note that the $\mathcal{E}^{\prime}$ so constructed depend only on the homomorphism $\mathcal{F}_{\mid C} \rightarrow \mathcal{L}^{\prime}$.

We now carry out this construction in details. First note that the restriction of $\mathcal{F}$ to $C$ is an extension of the degree $r_{1}=\frac{1}{2} I \cdot C+1$ sheaf $\mathcal{L}^{-1} \otimes \mathcal{O}_{C}\left(I_{\mid C}\right)$ by the degree $r_{2}=\frac{1}{2} I \cdot C-C^{2}-1$ sheaf $\mathcal{L}(-C)$. Accordingly, we will let $Y$ be the subset in the moduli space of stable rank two locally free sheaves on $C$ consisting of sheaves that are extensions of degree $r_{1}$ invertible sheaves by degree $r_{2}$ invertible sheaves. $Y$ is a quasi-projective scheme (with the reduced scheme structure). Let $y \in Y$ be any point and let $y \in U \subset Y$ be an (analytic or etále) neighborhood of $y \in Y$ such that there is a tautological family $\mathcal{V}_{U}$ on $C \times U$. By shrinking $U$ if necessary, we can assume that there is an integer $m$ such that $\left(\mathcal{V}_{U}\right)^{\vee}$ belongs to the exact sequence

$$
0 \longrightarrow\left(\mathcal{V}_{U}\right)^{\vee} \longrightarrow \mathcal{N}_{U} \longrightarrow \mathcal{T} \longrightarrow 0
$$

where $\mathcal{N}_{U}=\mathcal{O}_{C \times U}^{\oplus 2}\left(m p_{0} \times U\right)$ and $\mathcal{T}$ is a family of torsion sheaves on $C \times$ $U$ flat over $U$. Let $J=\operatorname{Pic}^{r_{1}}(C)$ (the component containing degree $r_{1}$ line bundles) and let $\mathcal{P}$ be the normalized Poincare line bundle on $C \times J$ (considered as a sheaf) so that $\mathcal{P}_{\mid p_{0} \times J} \cong \mathcal{O}_{J}$, where $p_{0} \in C$ is fixed. (For $\lambda \in J$, we will use $\mathcal{P}_{\lambda}$ to denote the restriction sheaf $\mathcal{P}_{\mid C \times\{\lambda\}}$.) Let $p_{C \times U}$, $p_{C \times J}$ and $p_{U \times J}$ be projections from $C \times U \times J$ to $C \times U, C \times J$ and $U \times J$ respectively. We consider the following direct image sheaves on $U \times J$ :

$$
\begin{gathered}
\mathcal{A}_{U \times J}=p_{U \times J *}\left(p_{C \times U}^{*} \mathcal{N}_{U} \otimes p_{C \times J}^{*} \mathcal{P}\right), \\
\mathcal{B}_{U \times J}=p_{U \times J *}\left(p_{C \times U}^{*} \mathcal{T} \otimes p_{C \times J}^{*} \mathcal{P}\right)
\end{gathered}
$$


and

$$
\mathcal{C}_{U \times J}=p_{U \times J *}\left(p_{C \times U}^{*}\left(\mathcal{V}_{U}\right)^{\vee} \otimes p_{C \times J}^{*} \mathcal{P}\right) .
$$

Both $\mathcal{A}_{U \times J}$ and $\mathcal{B}_{U \times J}$ are locally free. We let $\mathbf{A}$ be the vector bundle associated to $\mathcal{A}_{U \times J}$ and let $\mathcal{O}_{\mathbf{P}}(-1)$ be the tautological line bundle of the projective bundle $\mathbf{P}(\mathbf{A})$ of $\mathbf{A}$. (We use the convention adapted in §2.) Let $\pi: \mathbf{P}(\mathbf{A}) \rightarrow U \times J$ be the projection. Then composed with the induced homomorphism $\pi^{*} \mathcal{A}_{U \times J} \rightarrow \pi^{*} \mathcal{B}_{U \times J}$, we get

$$
f: \mathcal{O}_{\mathbf{P}}(-1) \longrightarrow \pi^{*} \mathcal{B}_{U \times J} .
$$

Since both $\mathcal{A}$ and $\mathcal{B}$ are locally free, for any $z \in \mathbf{P}(\mathbf{A})$ over $(y, \lambda) \in U \times J$, $z$ corresponds to a section (unique up to scalars) $\phi \in H^{0}\left(\mathcal{O}_{C}^{\oplus 2}\left(m p_{0}\right) \otimes \mathcal{P}_{\lambda}\right)$. Further, the restriction of $f$ to $\mathcal{O}_{\mathbf{P}}(-1) \otimes k(z)$ is trivial if and only if the image of $\phi$ in $H^{0}\left(\mathcal{T}_{\mid C \times\{y\}} \otimes \mathcal{P}_{\lambda}\right)$ is trivial. By the exactness of (3.3), this occurs exactly when $\phi$ can be lifted to a homomorphism $\tilde{\phi} \in \operatorname{Hom}_{C}\left(\mathcal{V}_{y}, \mathcal{P}_{\lambda}\right), \mathcal{V}_{y}=$ $\mathcal{V}_{U \mid C \times\{y\}}$. Hence, the set of all non-trivial homomorphisms $\operatorname{Horn}_{C}\left(\mathcal{V}_{y}, \mathcal{P}_{\lambda}\right)$ modulo scalars is parameterized by the scheme $f^{-1}(0) \subset \mathbf{P}(\mathbf{A})$. We let $\tilde{Z}$ be $f^{-1}(0)$ endowed with reduced scheme structure.

Lemma 3.2. (3.4) induces a homomorphism

$$
\beta_{\tilde{Z}}: \mathcal{O}_{\mathbf{P}}(-1)_{\mid \tilde{Z}} \longrightarrow\left(\pi^{*} \mathcal{C}_{U \times J}\right)_{\mid \tilde{Z}}
$$

so that for any closed $z \in \tilde{Z}$ over $(y, \lambda) \in U \times J$, the image

$$
\operatorname{Im}\left(\beta_{z}\right) \subset \operatorname{Hom}_{C}\left(\mathcal{F}_{y}, \mathcal{P}_{\lambda}\right)
$$

induced by the restriction homomorphism

$$
\beta_{z}: \mathcal{O}_{\mathbf{P}}(-1) \otimes k(z) \longrightarrow \pi^{*} \mathcal{C}_{U \times J} \otimes k(z) \longrightarrow \operatorname{Hom}_{C}\left(\mathcal{F}_{y}, \mathcal{P}_{\lambda_{1}}\right)
$$

is non-trivial.

Proof. Since $\pi$ is flat, $\pi^{*} \mathcal{A}_{U \times J}, \pi^{*} \mathcal{B}_{U \times J}$ and $\pi^{*} \mathcal{C}_{U \times J}$ belong to the exact sequence

$$
0 \longrightarrow \pi^{*} \mathcal{C}_{U \times J} \longrightarrow \pi^{*} \mathcal{A}_{U \times J} \stackrel{f}{\longrightarrow} \pi^{*} \mathcal{B}_{U \times J} .
$$

Because the composition of $\mathcal{O}_{\mathbf{P}}(-1)_{\mid \tilde{Z}} \rightarrow \pi^{*}\left(\mathcal{A}_{U \times J}\right)_{\mid \tilde{Z}}$ with $f$ is trivial, it lifts to a unique homomorphism $\beta_{\tilde{Z}}$ in (3.5). The non-triviality of $\operatorname{Im}\left(\beta_{z}\right)$ for $z \in \tilde{Z}$ is obvious. This proves the Lemma. 
In the following, we will use the section $\beta_{\tilde{Z}}$ to construct a family of homomorphisms of sheaves on $C \times \tilde{Z}$. Let $\pi_{C \times U}, \pi_{C \times J}$ and $\pi_{\tilde{Z}}$ be projections from $C \times \tilde{Z}$ to $C \times U, C \times J$ and $\tilde{Z}$ respectively. Then the section $\beta_{\tilde{Z}}$ provides us a homomorphism of sheaves

$$
\alpha_{\tilde{Z}}: \pi_{C \times U}^{*} \mathcal{V}_{U} \longrightarrow \pi_{\tilde{Z}}^{*}\left(\mathcal{O}_{\mathbf{P}}(1)_{\mid \tilde{Z}}\right) \otimes \pi_{C \times J}^{*} \mathcal{P}
$$

on $C \times \tilde{Z}$. Intuitively, $\beta_{\tilde{Z}}$ is the the one whose restriction to each $C \times\{z\} \subset$ $C \times \tilde{Z}$ is the image of $\beta_{z}$ in $\operatorname{Hom}_{C}\left(\mathcal{V}_{y}, \mathcal{P}_{\lambda}\right)$, where $z$ lies over $(y, \lambda) \in U \times J$. We denote the right hand side of (3.7) by $\mathcal{L}_{\tilde{Z}}$, which is an invertible sheaf on $C \times \tilde{Z}$. For technical reason, we let $Z \subset \tilde{Z}$ be the union of those irreducible components $A \subset \tilde{Z}$ such that the restriction of $\alpha_{\tilde{Z}}$ to general fibers of $C \times A \rightarrow A$ are surjective. We let $\alpha_{Z}$ and $\mathcal{L}_{Z}$ be restriction of $\alpha_{\tilde{Z}}$ and $\mathcal{L}_{\tilde{Z}}$ to $C \times Z$ respectively. We let $\Sigma \subset C \times Z$ be the subscheme so that $\operatorname{Im}\left(\alpha_{Z}\right)=\mathcal{L}_{Z} \otimes \mathcal{I}_{\Sigma}$, where $\mathcal{I}_{\Sigma}$ is the ideal sheaf of $\Sigma \subset C \times Z$. We now show that $\mathcal{I}_{\Sigma}$ is flat over $Z$.

Lemma 3.3. The ideal sheaf $\mathcal{I}_{\Sigma}$ is flat over $Z$.

Proof. Since $\mathcal{L}_{Z} \otimes \mathcal{I}_{\Sigma}$ is a quotient sheaf of the rank two locally free sheaf $\pi_{C \times U}^{*} \mathcal{V}, \Sigma$ is locally defined by at most two equations. Also by our choice of $Z, \Sigma$ does not meet general fibers of $C \times Z \rightarrow Z$ and does not contain any fiber of $C \times Z \rightarrow Z$. Therefore, $\operatorname{codim}(\Sigma) \geq 2$. Thus $\Sigma \subset C \times U$ is a local complete intersection scheme of codimension 2. Let $p \in \Sigma$ be any point. Since $\mathcal{I}_{\Sigma, p}$ is generated by two sections, say $f_{1}, f_{2}$, it belongs to the exact sequence

$$
0 \longrightarrow \mathcal{I}_{D_{1} \subset C \times Z, p} \longrightarrow \mathcal{I}_{\Sigma, p} \longrightarrow \mathcal{I}_{D_{2} \subset D_{1}, p} \longrightarrow 0,
$$

where $D_{1}$ is the divisor $\left\{f_{1}=0\right\}$ in $C \times Z$ and $D_{2}$ is the divisor $\left\{f_{2}=0\right\}$ in $D_{1}$. Here $\mathcal{I}_{D_{1} \subset C \times Z, p}$ and $\mathcal{I}_{D_{2} \subset D_{1}, p}$ are ideal sheaves of $D_{1} \subset C \times Z$ and $D_{2} \subset D_{1}$ at $p$ respectively, which are isomorphic to $\mathcal{O}_{C \times Z, p}$ and $\mathcal{O}_{D_{1}, p}$. Without loss of generality, we can assume $D_{1}$ is a divisor flat over $Z$ (because $\operatorname{supp}(\Sigma)$ contains no fibers of $C \times Z \rightarrow Z)$. Therefore, $\mathcal{I}_{D_{1} \subset C \times Z, p}$ and $\mathcal{I}_{D_{2} \subset D_{1}, p}$ are both flat over $Z$. Hence by (3.8), $\mathcal{I}_{\Sigma, p}$ is flat over $Z$ as desired.

We have the following lemma which states that the previous construction is independent of the choices made. 
Lemma 3.4. The scheme $\tilde{Z}=f^{-1}(0), Z \subset \tilde{Z}$ and the family (3.7) are independent of the choice of the inclusions $\left(\mathcal{V}_{U}\right)^{\vee} \rightarrow \mathcal{N}_{U}$.

Proof. We only need to check the following: Assume $\left(\mathcal{V}_{U}\right)^{\vee} \rightarrow \mathcal{O}_{C \times U}^{\oplus 2}\left(m^{\prime} p_{0} \times\right.$ $U$ ) is another inclusion with cokernel $\mathcal{T}^{\prime}$ flat over $U$ that makes the diagram

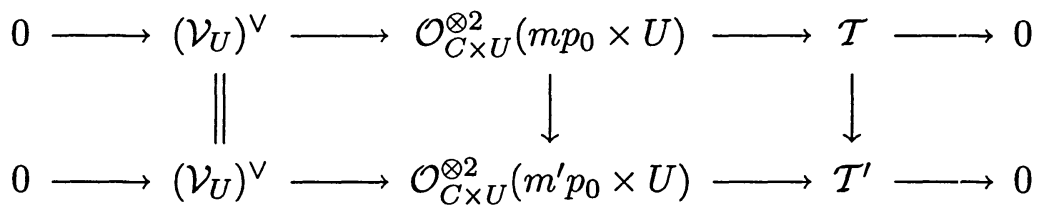

commutative and such that the vertical arrows are injective, and assume $Z \subset \tilde{Z} \subset \mathbf{P}(\mathbf{A})$ and $Z^{\prime} \subset \tilde{Z}^{\prime} \subset \mathbf{P}\left(\mathbf{A}^{\prime}\right)$ are the corresponding subschemes, then there is an isomorphism between $Z \subset \tilde{Z}$ and $Z^{\prime} \subset \tilde{Z}^{\prime}$ (non-canonically) and isomorphism between the corresponding pairs (3.7) (locally and noncanonically). This is obvious because $\mathbf{A}$ is a subbundle of $\mathbf{A}^{\prime}$, and that under the inclusion $\mathbf{P}(\mathbf{A}) \subset \mathbf{P}\left(\mathbf{A}^{\prime}\right)$ we have $\tilde{Z}^{\prime} \subset \mathbf{P}(\mathbf{A})$. We shall omit the details here.

Our next step is to introduce a subset of $Z$ that will provide us nonlocally free sheaves: We keep $p_{0} \in C$ and define $Z^{p_{0}} \subset Z$ be the the vanishing locus of the restriction to $\left\{p_{0}\right\} \times Z$ of (3.7):

$$
\alpha_{Z, p_{0}}: \pi^{*} \mathcal{V}_{0} \longrightarrow \mathcal{O}_{\mathbf{P}}(1)_{\mid Z}
$$

where $\pi: Z \rightarrow U$ is the projection and $\mathcal{V}_{0} \cong \mathcal{V}_{U \mid\left\{p_{0}\right\} \times U}$ is viewed as a sheaf on $U$. Note that $Z^{p_{0}} \subset Z$ has codimension at most two. Also, by our choice of $n_{0}$ in (1.1), $\operatorname{dim} \pi^{-1}(y)>10$ for any $y \in U$ [La, p45]. Since $\mathcal{O}_{\mathbf{P}}(1)$ is $\pi$-relatively ample [La,p46], and that $Z^{p_{0}} \cap \pi^{-1}(y)$ is the vanishing locus of two sections of the ample line bundle $\mathcal{O}_{\mathbf{P}}(1)_{\mid \pi^{-1}(y)}$, by the theorem on page 150 and remarks on page 152 of [GM] we can find a Riemannian metric on $Z$ so that

$$
H_{i}\left(\pi^{-1}(y)_{\mathrm{reg}}, Z^{p_{0}, \delta} \cap \pi^{-1}(y)_{\mathrm{reg}}\right)=0 \quad \text { for } i \leq 3,
$$

where the subscript reg stands for the regular locus of the set, and that $Z^{p_{0}, \delta}$ is the $\delta$-neighborhood of $Z^{p_{0}} \subset Z$.

We now extend this construction to $Y$. We cover $Y$ by (analytic or etále) open $U_{a}$ each of which admits a tautological family $\mathcal{V}_{a}$ fitting into the exact sequence (3.3) for some $m_{a}$. Then by the previous argument, we can find the family $Z_{a} \rightarrow U_{a}$, the family of homomorphisms

$$
\alpha_{Z_{a}}: \pi_{C \times U_{a}}^{*} \mathcal{V}_{a} \longrightarrow \pi_{Z_{a}}^{*}\left(\mathcal{O}_{\mathbf{P}_{a}}(1)_{\mid Z_{a}}\right) \otimes \pi_{C \times J}^{*} \mathcal{P}
$$


and subschemes $\Sigma_{a} \subset C \times Z_{a}$ and $Z_{a}^{p_{0}} \subset Z_{a}$. By Lemma 3.4, the schemes $Z_{a}$ glue together to form $Z \stackrel{\pi}{\longrightarrow} U$. Also over $C \times\left(U_{a} \cap U_{b}\right)$ we can find (non-canonical) isomorphism $\mathcal{V}_{a} \cong \mathcal{V}_{b}$ that will provide us isomorphism (noncanonical) over $Z_{a} \cap Z_{b}$ of the corresponding families (3.11). This guarantees that that the subschemes $\Sigma_{a}$ glue together to form a subscheme $\Sigma \subset C \times Z$ and that subschemes $Z_{a}^{p_{0}}$ will glue together to form a subscheme $Z^{p_{0}}$. Note that $Z \rightarrow Y$ and $Z^{p_{0}} \rightarrow Y$ are projective.

Now we construct the deformation needed to prove the vanishing of homology groups in Proposition 3.1. First, note that each $\mathcal{E} \in \mathcal{W}$ associates to a sheaf $\mathcal{F}_{\mathcal{E}}$ that is the kernel of $\mathcal{E} \rightarrow \mathcal{L}$, where $\mathcal{L}$ is the destabilizing quotient sheaf of $\mathcal{E}_{\mid C}$ (see (3.1)). ( $\mathcal{W}$ consists of sheaves in $\mathcal{Y}$ whose restriction to $C$ is locally free.) We let $e=-2 n_{0}^{2} H_{0}^{2}$ and

$$
W=\left\{\begin{array}{l|l}
\mathcal{E} \in \mathcal{W} \mid \begin{array}{l}
\mathcal{E} \text { is } 2 e \text {-stable, } \mathcal{F}_{\mathcal{E} \mid C} \text { is stable and the destabilizing } \\
\text { quotient sheaf of } \mathcal{E}_{\mid C} \text { has degree } \frac{1}{2} I \cdot C-1
\end{array}
\end{array}\right\} .
$$

We define $\rho: W \rightarrow Y$ to be the map sending $\mathcal{E} \in W$ to $\mathcal{F}_{\mathcal{E} \mid C}$. We now show that $\rho$ is a morphism: We first cover $W$ by (analytic or etále) open sets $W_{a}$ so that each of them admits tautological family $\mathcal{E}_{a}$ on $X \times W_{a}$. We let $\mathcal{E}_{a} \rightarrow \mathcal{L}_{a}$ be the quotient sheaf such that its restriction to each $C \times\{w\}$ is the destabilizing quotient sheaf of $\mathcal{E}_{a \mid C \times\{w\}}$. Let $\mathcal{F}_{a}$ be its kernel. Note that $\mathcal{F}_{a}$ belongs to the exact sequence

$$
0 \longrightarrow \mathcal{E}_{a}\left(-C \times W_{a}\right) \longrightarrow \mathcal{F}_{a} \longrightarrow \mathcal{L}_{a} \otimes p_{C}^{*} \mathcal{O}_{C}\left(I_{\mid C}\right) \longrightarrow 0,
$$

where $p_{C}: C \times W_{a} \rightarrow C$, similar to (3.2). Then the family $\mathcal{F}_{a \mid C \times W_{a}}$ will induce a morphism $\tilde{\rho}_{a}: W_{a} \rightarrow Y$. The collection $\tilde{\rho}_{a}$ 's glue together to form the morphism $\tilde{\rho}: W \rightarrow Y$. We let $A_{1}, \cdots, A_{k}$ be irreducible components of $W$ and let $Y^{\prime}$ be the disjoint union of $\tilde{\rho}\left(A_{i}\right): Y^{\prime}=\coprod_{i=1}^{k} \tilde{\rho}\left(A_{i}\right)$. Now let $W^{\prime}=W-\cup_{i \neq j}\left(A_{i} \cap A_{j}\right)$. Then $\tilde{\rho}$ induces a morphism $\rho: W^{\prime} \rightarrow Y^{\prime}$ whose restriction to $A_{i}-\cup_{j \neq i} A_{j}$ factor through $\tilde{\rho}\left(A_{i}\right) \subset Y^{\prime}$. Let $Z^{\prime}=Y^{\prime} \times_{Y} Z$ with $\pi^{\prime}$ the first projection and let $Z_{\text {reg }}^{\prime}$ be the Zariski open subset of $Z^{\prime}$ over which $\pi^{\prime}: Z^{\prime} \rightarrow Y^{\prime}$ is smooth. Next we let $Y_{0} \subset Y^{\prime}$ be a Zariski open subset such that $Z_{0} \equiv \pi^{\prime-1}\left(Y_{0}\right), Z_{0, \text { reg }} \equiv \pi^{\prime-1}\left(Y_{0}\right) \cap Z_{\text {reg }}^{\prime}$ and $Z_{0}^{p_{0}} \equiv \pi^{\prime-1}\left(Y_{0}\right) \cap Z^{p_{0}}$ are topological fiber bundles over $Y_{0}$ :

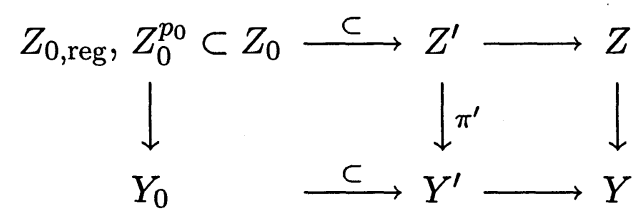


Such $Y_{0}$ can be found as follows: Since $\pi^{\prime}: Z^{\prime} \rightarrow Y^{\prime}$ is projective, there are Whitney stratification $\mathcal{S}_{Z^{\prime}}$ of $Z^{\prime}$ and $\mathcal{S}_{Y^{\prime}}$ of $Y^{\prime}$ by algebraic subvarieties such that $\pi^{\prime}$ is a stratified map (see [GM] for definition). Without loss of generality, we can assume $Z_{\mathrm{reg}}^{\prime}$ and $Z^{p_{0}} \cap Z^{\prime}$ are union of strata of $\mathcal{S}_{Z^{\prime}}$. Let $Y_{0}$ be the union of open strata in $Y^{\prime}$ and let $Z_{0}=\pi^{\prime-1}\left(Y_{0}\right) . Y_{0} \subset Y^{\prime}$ must be dense. Then by Thom's first isotopy lemma, $Z_{0}, Z_{0, \text { reg }} \equiv Z_{0} \cap Z_{\text {reg }}^{\prime}$ and $Z_{0}^{p_{0}} \equiv Z_{0} \cap Z^{p_{0}}$ are topological fiber bundles over $Y_{0}$. We let $W_{0}=\rho^{-1}\left(Y_{0}\right)$. Finally we form the fiber product

$$
V=Z_{0} \times_{Y_{0}} W_{0},
$$

and its subspaces $V_{\text {reg }}=Z_{0 \text {,reg }} \times_{Y_{0}} W_{0}$ and $V^{p_{0}}=Z_{0}^{p_{0}} \times_{Y_{0}} W_{0}$.

Remark 3.5. The reason we choose $Y^{\prime}$ to be the disjoint union of $\tilde{\rho}\left(A_{i}\right)$ is because in doing so we can be sure that $W_{0} \subset W$ is dense. Note that this may not necessarily be the case if we use $Y$ rather than $Y^{\prime}$ when some $\tilde{\rho}\left(A_{i}\right)$ is a proper subvariety of $\tilde{\rho}\left(A_{j}\right)$.

Our next step is to form a family of sheaves over $V$ that will be the deformations promised at the beginning of this section. We still work with a covering $W_{a}$ of $W_{0}$ and families $\mathcal{E}_{a}$ and $\mathcal{F}_{a}$. By shrinking $W_{a}$ if necessarily, we can assume $\rho\left(W_{a}\right)$ is contained in $U_{b} \subset Y_{0}$ for some $b$, where $U_{b}$ is an open set satisfying conditions spelled out before (3.3). Let

$$
\alpha_{Z_{b}}: \pi_{C \times U_{b}}^{*} \mathcal{V}_{b} \longrightarrow \mathcal{L}_{b} \otimes \mathcal{I}_{\Sigma_{b}}
$$

be the family of homomorphism as in (3.11). By shrinking $W_{a}$ further if necessarily, we can assume that there is an isomorphism

$$
\phi_{a}: \mathcal{F}_{a \mid C \times W_{a}} \stackrel{\cong}{\longrightarrow}\left(\left(1_{C} \times \rho_{b a}\right)^{*} \mathcal{V}_{b}\right)_{\mid C \times W_{a}}
$$

where $\rho_{b a}=\rho_{\mid W_{a}}: W_{a} \rightarrow U_{b}$. Then composed with $\alpha_{Z_{b}}$ in (3.14), we get a surjective homomorphism

$$
\varphi:\left(1_{X} \times \pi_{W_{0}}\right)^{*} \mathcal{F}_{a} \longrightarrow\left(1_{C} \times \pi_{Y_{0}}\right)^{*} \mathcal{V}_{b} \longrightarrow\left(1_{C} \times \pi_{Y_{0}}\right)^{*}\left(\mathcal{L}_{b} \otimes \mathcal{I}_{\Sigma_{b}}\right)
$$

of sheaves on $V_{a}=Z_{0} \times_{Y_{0}} W_{a}$, where $\pi_{W_{0}}$ is the second projection of $V_{a}$ and $\pi_{Y_{0}}: V_{a} \rightarrow Y_{0}$ is the obvious projection. Let $\mathcal{G}_{a}$ be the kernel of this homomorphism: Namely

$$
0 \longrightarrow \mathcal{G}_{a} \longrightarrow\left(1_{X} \times \pi_{W_{0}}\right)^{*} \mathcal{F}_{a} \stackrel{\varphi}{\longrightarrow}\left(1_{C} \times \pi_{Y_{0}}\right)^{*}\left(\mathcal{L}_{b} \otimes \mathcal{I}_{\Sigma_{b}}\right) \longrightarrow 0
$$

is exact. Since (3.14) is surjective and $\mathcal{L}_{b} \otimes \mathcal{I}_{\Sigma_{b}}$ is flat over $Y_{b}$ (Lemma $3.3), \mathcal{G}_{a}$ is a family of torsion free sheaves on $X \times V_{a}$. Also since sheaves 
in $W_{0}$ are $2 e-H$-stable, $\mathcal{G}_{a}$ is indeed a family of $H$-stable sheaves on $X$. From the construction, it is clear that member of the family $\mathcal{G}_{a}$ tensored by $\varnothing_{X \times V_{a}}\left(C \times V_{a}\right)$ has determinant $I$ and second Chern class $d$. Thus it induces a morphism $\eta_{a}: V_{a} \rightarrow \mathfrak{M}_{H}(I, d)$ by the universality of the moduli scheme. By Lemma 3.4, morphisms $\eta_{a}$ glue together to form a morphism $\eta: V \rightarrow \mathfrak{M}_{H}(I, d)$. We list the properties of the family $V \stackrel{\pi_{W_{0}}}{\longrightarrow} W_{0}$ and the morphism $\eta: V \rightarrow \mathfrak{M}_{H}(I, d)$ in the following proposition.

Proposition 3.6. Let $H_{0}, H_{0} \in \mathcal{C} \Subset N S_{\mathbb{Q}}^{+}, C \subset X$ and $N$ be given at the beginning of this section. Then for any $d \geq N$ and $\left(H_{0}, I, d\right)$-suitable $H \in \mathcal{C}$, the subset $\mathcal{W} \subset \mathfrak{M}_{H}(I, d)$ has pure codimension of $g+1$, where $g=g(C)$, and is locally irreducible. Further, the family $V \stackrel{W_{0}}{\longrightarrow} W_{0}$ has the following properties:

(1) $\eta(V) \subset \overline{\mathcal{W}}$, where $\overline{\mathcal{W}}$ is the closure of $\mathcal{W} \subset \mathfrak{M}_{H}(I, d)$;

(2) If $\mathcal{E} \in W_{0}$, then all $2 e-H$-stable sheaves $\mathcal{E}^{\prime} \in \mathfrak{M}_{H}(I, d)$ satisfying $\mathcal{E}_{\mid 2 C} \cong \mathcal{E}_{\mid 2 C}^{\prime}$ belong to $W_{0}$ as well;

(3) There is a section $s: W_{0} \rightarrow V$ (of $V \stackrel{\pi_{W_{0}}}{\longrightarrow} W_{0}$ ) such that $\eta \circ s$ coincides with the inclusion $W_{0} \subset \mathfrak{M}_{H}(I, d)$. Further, if we let $W^{0}=$ $s^{-1}\left(V_{\text {reg }}\right)$ then $W^{0}$ is dense in $\mathcal{W}$ and $W^{0} \cap \mathcal{S} \mathfrak{M}_{H}(I, d)$ is dense in $\overline{\mathcal{W}} \cap \mathcal{S M}_{H}(I, d)$;

(4) Both $V^{p_{0}}$ and $V_{\text {reg }}$ are topological bundles over $W_{0}$. Further, if we let $V^{p_{0}, \delta}=\left(Z^{p_{0}, \delta} \cap Z_{0}\right) \times_{Y_{0}} W_{0}$, then for any $w \in W_{0}$ the pair

$$
\left(V_{\text {reg }} \cap \pi_{W_{0}}^{-1}(w), V^{p_{0}, \delta} \cap V_{\text {reg }} \cap \pi_{W_{0}}^{-1}(w)\right)
$$

is homologically 3-connected;

(5) We have $\eta\left(V^{p_{0}}\right) \subset \mathcal{S M}_{H}(I, d)$. Also for any $w \in W_{0}$ associated to non-locally free sheaf, $\eta\left(\pi_{W_{0}}^{-1}(w)\right) \subset \mathcal{S} \mathfrak{M}_{H}(I, d)$.

Proof. We first prove that $\mathcal{W}$ has pure codimension $g+1$. Let $p \in \mathcal{W}$ be any point corresponding to $\mathcal{E}$ and let $U \subset \mathfrak{M}_{H}(I, d)$ be an analytic neighborhood of $p$ so that the restriction of the local tautological family $\mathcal{E}_{U}$ to $C \times U$ induces a map

$$
\varphi:(U, p) \longrightarrow(A, 0)
$$

provided by Definition 2.3, where $(A, 0)$ is the versal deformation space of $\mathcal{E}_{\mid C}$ of fixed determinant. Since $\varphi^{-1}\left(A_{0}\right)=U \cap \mathcal{W}$ and $\operatorname{codim}\left(A_{0}, A\right)=g+1$, 
the codimension of each component of $\mathcal{W}$ at $p$ is at most $g+1$. However, when $p \notin \Lambda_{1}^{C}$ (cf. (1.3)), $\varphi$ is a submersion at $p$ and the codimension of $\mathcal{W}$ at $p$ is exactly $g+1$. On the other hand, $\operatorname{dim} \Lambda_{1}^{C} \leq \operatorname{dim} \mathfrak{M}_{H}(I, d)-3 g$, by Lemma 1.5 and $\operatorname{codim} \mathcal{W}>3 g$, by Lemma 1.7 (3). Thus $\mathcal{W}-\Lambda_{1}^{C}$ is dense in $\mathcal{W}$ and therefore $\mathcal{W}$ has pure codimension $g+1$ as claimed. Next, we show that $\mathcal{W}$ is locally irreducible. Let $T \subset \mathcal{W}$ be the subset over which $\mathcal{W}$ is not locally irreducible. Since $\mathfrak{M}_{H}(I, d)$ is a local complete intersection and $\mathcal{W} \subset \mathfrak{M}_{H}(I, d)$ is defined by $3 g-2$ equations (Lemma 1.7), $T$ is either empty or $\operatorname{codim}\left(T, \mathfrak{M}_{H}(I, d)\right)$ can at most be $2 g+1$, by (2) of Lemma 1.12. Thus if $T \neq \emptyset$ then $T-\Lambda_{1}^{C} \neq \emptyset$. Let $p \in T-\Lambda_{1}^{C}$ be corresponding to $\mathcal{E}$ and let $p \in U \subset \mathfrak{M}_{H}(I, d)$ be an analytic neighborhood so that we have the map (3.17) provided by Definition 2.3, where $(A, 0)$ is the versal deformation space of $\mathcal{E}_{\mid C}$ of fixed determinant. Since $\varphi$ is a submersion at $p$ and $A_{0} \subset A$ is locally irreducible by Proposition $2.2, U \cap \mathcal{W}=\varphi^{-1}\left(A_{0}\right)$ will be locally irreducible at $p$ as well. This contradicts to the assumption that $p \in T$. Hence $T=\emptyset$ and thus $\mathcal{W}$ is locally irreducible.

Next, we prove the properties listed in the proposition. First, by restricting the exact sequence (3.16) to $C \times\{v\}$, where $v \in V_{a}$ is any general element, we see that $\mathcal{G}_{a \mid C \times\{v\}}(C)$ is an extension of a degree $\frac{1}{2} I \cdot C-1$ invertible sheaf by a degree $\frac{1}{2} I \cdot C+1$ invertible sheaf. Hence $\eta(v) \in \mathcal{W}$ and thus $\eta(V) \subset \overline{\mathcal{W}}$. This proves (1).

The property (2) follows from the fact that if $\mathcal{E}$ and $\mathcal{E}^{\prime} \in W$ such that $\mathcal{E}_{\mid C} \cong \mathcal{E}_{\mid C}^{\prime}$, then $\mathcal{F}_{\mathcal{E} \mid C} \cong \mathcal{F}_{\mathcal{E}^{\prime} \mid C}$.

Now we prove (3). Over $W_{a}$, the sheaf $\mathcal{L}_{a} \otimes p_{C}^{*} \mathcal{O}_{C}\left(I_{\mid C}\right)$ in (3.13) will induce a morphism $\xi: W_{a} \rightarrow \operatorname{Pic}^{r_{1}}(C)$ and the homomorphism $\varphi$ in (3.16) will induce a section of the sheaf $(\rho \times \xi)^{*} \mathcal{C}_{U_{b} \times J}$. Here we have assumed that $\rho\left(W_{a}\right) \subset U_{b}$. Combined with (3.6), we obtain a section of the locally free sheaf $(\rho \times \xi)^{*} \mathcal{A}_{U_{b} \times J}$, which will provide us the desired section $s_{a}: W_{a} \rightarrow$ $\tilde{Z} \times_{Y} W_{a}$. This section factor through $Z \times_{Y} W_{a}$ because the $\varphi$ in (3.16) is surjective. Further because we can recover $\mathcal{G}_{a}$ from the $\varphi$ in (3.16i) and vice versa, $\eta \circ s_{a}=$ id and $s_{a} \circ \eta(v)=v$ if $\eta(v) \in W_{0}$. Therefore by Lemma 3.4, $s_{a}$ 's glue together to form the desired section $s: W \rightarrow V$. Note that property (2) still holds for $W^{0}$.

To complete the proof of (3), we need to check the density of: $W^{0} \subset \mathcal{W}$ and of $W^{0} \cap \mathcal{S M}_{H}(I, d) \subset \overline{\mathcal{W}} \cap \mathcal{S M}_{H}(I, d)$. Note that $W_{0} \subset W$ is dense by Remark 3.5. Now we show that $W \subset \mathcal{W}$ is dense. Let $\mathcal{E} E \mathcal{W}$ be a general sheaf. By Lemma 1.3, $\mathcal{E}$ is $2 e$-stable and $\operatorname{Ext}^{2}(\mathcal{E}, \mathcal{E}(-C))^{0}=0$. Let $\mathcal{L}$ be the destabilizing quotient sheaf of $\mathcal{E}_{\mid C}$ and $\mathcal{F}$ be the kernel of $\mathcal{E} \rightarrow \mathcal{L}$. Then $\mathcal{F}_{\mid C}$ is the extension of $\mathcal{L}^{-1} \otimes \mathcal{O}_{C}\left(I_{\mid C}\right)$ by $\mathcal{L}(-C)$. Let $\mathcal{V}_{s}, s \in S$ is an 
affine curve, be a deformation of $\mathcal{F}_{\mid C}$ such that each $\mathcal{V}_{s}$ belongs to the exact sequence

$$
0 \longrightarrow \mathcal{L}(-C) \longrightarrow \mathcal{V}_{s} \stackrel{\beta}{\longrightarrow} \mathcal{L}^{-1} \otimes \mathcal{O}_{C}\left(I_{\mid C}\right) \longrightarrow 0
$$

and for general $s \in S$ the sheaf $\mathcal{V}_{s}$ is stable. Since $\operatorname{Ext}^{2}(\mathcal{E}, \mathcal{E}(-2 C))^{0}=0$, we have $\operatorname{Ext}^{2}(\mathcal{F}, \mathcal{F}(-C))^{0}=0$. Hence there is a family $\mathcal{F}_{s}, s \in S$, of sheaves on $X$ such that $\mathcal{F}_{s \mid C} \cong \mathcal{V}_{s}$. Using the $\beta$ in (3.18), we have a surjective homomorphism $\mathcal{F}_{s} \rightarrow \mathcal{V}_{s} \rightarrow \mathcal{L}^{-1} \otimes \mathcal{O}_{C}\left(I_{\mid C}\right)$ whose kernel we denote by $\mathcal{E}_{s}$. It is easy to check that $\mathcal{E}_{s}$ is a deformation of $\mathcal{E}$ and for general $s \in S$ the sheaf $\mathcal{E}_{s} \in W$ because $\mathcal{F}_{s}$ is stable. This shows that $W$ is dense in $\mathcal{W}$.

To show that $W^{0} \subset \mathcal{W}$ is dense it suffices to show that $W^{0} \cap A \neq \emptyset$ for each irreducible component $A$ of $W_{0}$. Let $\mathcal{E} \in A$ be a general element and let $v \in \pi_{W_{0}}^{-1}(\mathcal{E}) \cap V_{\text {reg }}$ be a general element, where $\pi_{W_{0}}: V \rightarrow W_{0}$. Then $\eta(v) \in W^{0}$ since $s \circ \eta(v)=v$. $\eta(v)$ will be in $A$ if we choose $v$ so that $v$ and $s(\mathcal{E})$ are in the same irreducible component of $\pi_{W_{0}}^{-1}(\mathcal{E})$, which is possible because $V_{\text {reg }} \rightarrow W_{0}$ and $V \rightarrow W_{0}$ are stratified maps, and $V_{\text {reg }}$ is dense in $V$ and $\mathcal{E} \in A$ is general. Thus $W^{0} \cap A \neq \emptyset$. This proves $W^{0} \subset \mathcal{W}$ is dense. Next we prove

$$
W^{0} \cap \mathcal{S M}_{H}(I, d) \subset \overline{\mathcal{W}} \cap \mathcal{S} \mathfrak{M}_{H}(I, d)
$$

is dense. By dimension count, it is clear that $\left(\overline{\mathcal{W}}-\Lambda_{1}^{C}\right) \cap \mathcal{S M}_{H}(I, d)$ is dense in $\overline{\mathcal{W}} \cap \mathcal{S M}_{H}(I, d)$. Let $\mathcal{E} \in\left(\overline{\mathcal{W}}-\Lambda_{1}^{C}\right) \cap \mathcal{S} \mathfrak{M}_{H}(I, d)$ be a general sheaf . Because $H^{2}\left(\mathcal{E} n d^{0}(\mathcal{E})(-2 C)\right)=0$, following the deformation argument in [GL, p86-87] we see that the subset in $\overline{\mathcal{W}}$ consisting of sheaves non-locally free along $C$ has codimension at least 2 in $\overline{\mathcal{W}}$. Therefore $\mathcal{E}_{\mid C}$ is locally free because $\mathcal{E}$ is general in $\overline{\mathcal{W}} \cap \mathcal{S} \mathfrak{M}_{H}(I, d)$ and that $\mathcal{S M}_{H}(I, d) \subset \mathfrak{M}_{H}(I, d)$ is a divisor. It remains to show that $\mathcal{E} \in W^{0}$. Because $W^{0} \subset \mathcal{W}$ is dense, we can find deformation $\mathcal{F}_{t}$ of $\mathcal{E}$ such that $\mathcal{F}_{t} \in W^{0}$ for general $t$. Because $H^{0}\left(\mathcal{E} n d^{0}(\mathcal{E})(-2 C)\right)=0$, we can find deformation $\mathcal{E}_{t}$ of $\mathcal{E}$ such that $\mathcal{F}_{t \mid 2 C} \cong$ $\mathcal{E}_{t \mid 2 C}$ and $\mathcal{E}_{t} \in \mathcal{S} \mathfrak{M}_{H}(I, d)$, following the same deformation argument in [GL]. Because (2) holds for $W^{0}, \mathcal{E}_{t} \in W^{0} \cap \mathcal{S} \mathfrak{M}_{H}(I, d)$. This proves (3).

The property (4) follows from (3.10) and the fact that $Z_{0}, Z_{0}^{p_{0}}$ and $Z_{0, \text { reg }}$ are fiber bundles over $Y_{0}$. The second statement of (5) is apparent because if $w \in W$ associates to a non-locally free sheaf, say $\mathcal{E}$, then $\mathcal{E}$ is non-locally free away from $C$. However, all sheaves in $\eta\left(\pi_{W_{0}}^{-1}(w)\right)$ are isomorphic to $\mathcal{E}$ over $X-C$. Thus they must belong to $\mathcal{S} \mathfrak{M}_{H}(I, d)$. Now let $v \in V^{p_{0}}$ be over a point in $W_{a}$. Then the restriction of (3.16) to $X \times\{v\}$ :

$$
0 \rightarrow \mathcal{G}_{a \mid X \times\{v\}} \rightarrow\left(1_{C} \times \pi_{Y_{0}}\right)^{*} \mathcal{V}_{b \mid X \times\{v\}} \rightarrow\left(1_{C} \times \pi_{Y_{0}}\right)^{*}\left(\mathcal{L}_{b} \otimes \mathcal{I}_{\Sigma_{b}}\right)_{\mid X \times\{v\}} \rightarrow 0
$$


is exact. Since the third (non-zero) term above has torsion supported at $p_{0} \in X, \mathcal{G}_{a \mid X \times\{v\}}$ is non-locally free at $p_{0}$. Hence $\eta(v) \in \mathcal{S M}_{H}(I, d)$. This completes the proof of the proposition.

In the following, we will show how to use the pair $\left(W^{0}, Z_{0}\right)$ to get the desired vanishing result in Proposition 3.1. We begin with the following lemma:

Lemma 3.7. Let the notation be as before, then for $i \leq 2$,

$$
H_{i}\left(W^{0}, W^{0} \cap \mathcal{S} \mathfrak{M}_{H}(I, d)\right) \longrightarrow H_{i}\left(\overline{\mathcal{W}}, \overline{\mathcal{W}} \cap \mathcal{S} \mathfrak{M}_{H}(I, d)\right)
$$

is trivial. (i.e. its image is $\{0\}$.)

Proof. Let $\xi \in H_{i}\left(W^{0}, W^{0} \cap \mathcal{S M}_{H}(I, d)\right)$ be any element represented by a cycle $(\Sigma, \partial \Sigma) \subset\left(W^{0}, W^{0} \cap \mathcal{S} \mathfrak{M}_{H}(I, d)\right)$. By using section $s: W^{0} \rightarrow V_{\text {reg }}$ we get a cycle $(s(\Sigma), \partial s(\Sigma)) \subset\left(V_{\text {reg }}, V_{\text {reg }} \cap \eta^{-1}\left(\mathcal{S M}_{H}(I, d)\right)\right)$. Because of (3) and (4) in Proposition 3.6, for each $0<\delta \ll 1$ we can find an $(i+1)$-chain $T^{\prime} \subset V_{\text {reg }}$ such that the boundary $\partial T^{\prime}$ is the union of $s(\Sigma)$ with $A_{1}^{\prime}$ and $A_{2}^{\prime}$, where $A_{1}^{\prime} \subset V_{\text {reg }} \cap\left(Z^{p_{0}, \delta} \times_{Y} W_{0}\right)$ and

$$
A_{2}^{\prime} \subset \bigcup\left\{\pi_{W_{0}}^{-1}(w) \mid w \in s(\partial \Sigma)\right\} \subset \eta^{-1}\left(\mathcal{S M}_{H}(I, d)\right) .
$$

Because $V \rightarrow W_{0}$ is proper and $\delta$ is arbitrarily small, we can find $T \subset V$ so that $\partial T=\Sigma^{\prime} \cup A_{1} \cup A_{2}$ with $A_{1} \subset V^{p_{0}}$ and $A_{2} \subset \eta^{-1}\left(\mathcal{S}_{H}(I, d)\right)$. Thus $\partial(\eta(T))=\Sigma$ modulo $\mathcal{S M}_{H}(I, d)$. In particular, the image of $\xi$ in $H_{i}\left(\overline{\mathcal{W}}, \overline{\mathcal{W}} \cap \mathcal{S} \mathfrak{M}_{H}(I, d)\right)$ is trivial.

Next we show that (3.19) is surjective. We remark that we will use ordinary topology in the rest of this section unless otherwise is mentioned. Note that this will prove Proposition 3.1. We first divide $\overline{\mathcal{W}}-W^{0}$ into two subsets: We let $B_{1}$ be $\left(\overline{\mathcal{W}}-W^{0}\right) \cap \mathcal{S M}_{H}(I, d)$ and let $B_{2}=(\overline{\mathcal{W}}-$ $\left.W^{0}\right)-\mathcal{S} \mathfrak{M}_{H}(I, d)$. Since $\mathfrak{M}_{H}(I, d)$ is quasi-projective, there is a Whitneystratification of $\mathfrak{M}_{H}(I, d)$ so that the subsets $\overline{\mathcal{W}}, W^{0}, \mathcal{S} \mathfrak{M}_{H}\left(I, d^{\prime}\right), \Lambda_{1}^{C}, B_{1}$ and $B_{2}$ are unions of strata. To simplify the notation, in the following we will denote $\mathcal{W}_{1}=\overline{\mathcal{W}}-B_{1}$ and $\mathcal{W}_{2}=\mathcal{W}_{1}-B_{2}$. Note that $\mathcal{W}_{2}=W^{0}$.

We first show that

$$
H_{i}\left(\mathcal{W}_{1}, \mathcal{W}_{1} \cap \mathcal{S} \mathfrak{M}_{H}(I, d)\right) \longrightarrow H_{i}\left(\overline{\mathcal{W}}, \overline{\mathcal{W}} \cap \mathcal{S} \mathfrak{M}_{H}(I, d)\right), \quad i \leq 2
$$

is surjective. Let $K_{1}, \ldots, K_{n}$ be open strata of $B_{1}$ and let $K^{--}=B_{1}-$ $\cup_{j=1}^{n} K_{j}$. Since $\overline{\mathcal{W}}$ is locally irreducible away from $\mathcal{S}_{H}(I, d)$ and has pure 
codimension $g+1$, and that $\mathcal{S M}_{H}(I, d) \subset \mathfrak{M}_{H}(I, d)$ is a Cartier divisor, by the furthermore part of Lemma 1.13,

$H_{i}\left(\overline{\mathcal{W}}-K^{-},\left(\overline{\mathcal{W}}-K^{-}\right) \cap \mathcal{S} \mathfrak{M}_{H}(I, d)\right) \longrightarrow H_{i}\left(\overline{\mathcal{W}}, \overline{\mathcal{W}} \cap \mathcal{S} \mathfrak{M}_{H}(I, d)\right), \quad i \leq 2$

is surjective, $\operatorname{since} \operatorname{codim}\left(K^{-}, \overline{\mathcal{W}} \cap \mathcal{S} \mathfrak{M}_{H}(I, d)\right) \geq 2$. Let $V_{l}=\overline{\mathcal{W}}-K^{-}-$ $\cup_{j=1}^{l} K_{j}$. To prove the surjectivity of (3.21), it suffices to show that

$$
H_{i}\left(V_{l+1}, V_{l+1} \cap \mathcal{S} \mathfrak{M}_{H}(I, d)\right) \longrightarrow H_{i}\left(V_{l}, V_{l} \cap \mathcal{S} \mathfrak{M}_{H}(I, d)\right), \quad i \leq 2
$$

is surjective for $l=1, \cdots, n$. Now let $\Delta_{l} \subset K_{l}$ be any non-empty compact subset. Then by Lemma 1.14, we have

$H_{i}\left(V_{l+1} \cup \Delta_{l},\left(V_{l+1} \cup \Delta_{l}\right) \cap \mathcal{S M}_{H}(I, d)\right) \longrightarrow H_{i}\left(V_{l}, V_{l} \cap \mathcal{S M}_{H}(I, d)\right), \quad i \leq 2$

is surjective. Now we show that the image of

$$
H_{i}\left(V_{l+1}, V_{l+1} \cap \mathcal{S M}_{H}(I, d)\right) \longrightarrow H_{i}\left(V_{l}, V_{l} \cap \mathcal{S} \mathfrak{M}_{H}(I, d)\right), \quad i \leq 2
$$

is the same as the image of (3.22). The strategy in proving this is to find an analytic component of $\mathcal{S M}_{H}(I, d)$, say $U$, such that $U$ intersects $K_{l}$ transversally at some points. Then if we choose $\Delta_{l}$ close to $U \cap K$, we can show that (3.22) and (3.23) have same images.

Now we provide the details of this argument. Let $\mathcal{E} \in K_{l}$ be a general point. Because $\operatorname{codim}\left(K_{l}\right)=g+3, \mathcal{E}$ is $2 e-H$-stable and

$$
H^{2}\left(\mathcal{E} n d^{0}(\mathcal{E})(-2 C)\right)=0 .
$$

We claim that either $\mathcal{E}_{\mid C}$ is not locally free and $\ell\left(\mathcal{E}^{\vee \vee} / \mathcal{E}\right)=1$, or $\mathcal{E}_{\mid C}$ is locally free and $\mathcal{E} \in \mathcal{S}_{1} \mathfrak{M}_{H}(I, d) \cup \mathcal{S}_{2}^{0} \mathfrak{M}_{H}(I, d)$. Indeed, following the proof of (3) of Proposition 3.6, we know that the set of $\mathcal{E} \in \overline{\mathcal{W}}$ that are nonlocally free along $C$ has codimension at least 2 in $\overline{\mathcal{W}}$. For the same reason, the set of $\mathcal{E} \in \overline{\mathcal{W}}$ that are non-locally free along $C$ and have $\ell\left(\mathcal{E}^{\vee \vee} / \mathcal{E}\right) \geq 2$ has codimension at least 3 in $\overline{\mathcal{W}}$. Therefore if $\mathcal{E}_{\mid C}$ is not locally free then $\ell\left(\mathcal{E}^{\vee \vee} / \mathcal{E}\right)=1$. When $\mathcal{E}_{\mid C}$ is locally free, then $\mathcal{E} \in \mathcal{S}_{1} \mathfrak{M}_{H}(I, d) \cup \mathcal{S}_{2}^{0} \mathfrak{M}_{H}(I, d)$, because its compliment in $\mathcal{W} \cap \mathcal{S M}_{H}(I, d)$ has codimension 3 in $\mathcal{W}$. Next, we estimate $\operatorname{dim} \operatorname{Ext}_{2 C}^{0}\left(\left(\mathcal{E}^{\vee \vee}\right)_{\mid 2 C},\left(\mathcal{E}^{\vee \vee}\right)_{\mid 2 C}\right)^{0}$. When $\mathcal{E}_{\mid C}$ is not locally free, we claim that the destabilizing quotient sheaf of $\left(\mathcal{E}^{\vee \mathrm{V}}\right)_{\mid C}$ has degree at least $\frac{1}{2} I \cdot C-3$. If not, then proof of Lemma 2.7 we can find deformation $\mathcal{E}_{t}$ of $\mathcal{E}$ so that $\mathcal{E}_{t \mid C}$ is locally free and has degree $\frac{1}{2} I \cdot C-2$ destabilizing quotient sheaf for general $t$. Then $K_{l}$ will be contained in the closure of $\mathcal{W}_{0}$ in $\mathfrak{M}_{H}(I, d)$, where $\mathcal{W}_{0}$ consists of $\mathcal{F}$ such that $\mathcal{F}_{\mid C}$ is locally free and has degree $\frac{1}{2} I \cdot C-2$ 
destabilizing quotient sheaf. Since sheaves in $K_{l}$ are non-locally free along $C, K_{l} \subset \overline{\mathcal{W}}_{0}$ has codimension at least 2. (See proof of (3) of Proposition 3.6.) However, $\overline{\mathcal{W}}_{0} \subset \overline{\mathcal{W}}$ has codimension at least 1 . Hence the codimension of $K_{l}$ in $\mathfrak{M}_{H}(I, d)$ will be bigger than $g+3$. A contradiction. For the same reason, if $\mathcal{E}_{\mid C}$ is locally free, then the destabilizing quotient sheaf: of $\mathcal{E}_{\mid C}$ has degree at least $\frac{1}{2} I \cdot C-3$. In particular, we have

$$
\operatorname{dim} \operatorname{Ext}_{2 C}^{0}\left(\left(\mathcal{E}^{\vee \vee}\right)_{\mid 2 C},\left(\mathcal{E}^{\vee \vee}\right)_{\mid 2 C}\right)^{0} \leq 2 \operatorname{dim} \operatorname{Ext}^{0}\left(\left(\mathcal{E}^{\vee \vee}\right)_{\mid C},\left(\mathcal{E}^{\vee \vee}\right)_{\mid C}\right)^{0} \leq 18 .
$$

Now let $a=\ell\left(\mathcal{E}^{\vee \vee} / \mathcal{E}\right)$. Note that $a=1$ or 2 . We consider the set

$$
Q=\left\{\mathcal{F} \in \mathfrak{M}_{H}(I, d-a) \mid \mathcal{F} \text { locally free and } \mathcal{F}_{\mid 2 C} \cong\left(\mathcal{E}^{\vee \vee}\right)_{\mid 2 C}\right\}
$$

and the set

$$
Q_{K_{l}}=\left\{\mathcal{F} \in Q \mid \text { there is an } \mathcal{E}^{\prime} \in K_{l} \text { such that } \mathcal{E}^{\prime} \text { is a subsheaf: of } \mathcal{F}\right\} .
$$

We claim that $Q_{K_{l}} \subset Q$ contains a neighborhood of $\mathcal{E}^{\vee \vee} \in Q$. Indeed, let $\mathcal{F}_{t}$ be any deformation of $\mathcal{E}^{\vee \vee}$ in $Q$. In case $\mathcal{E}_{\mid C}$ is locally free, then $\mathcal{F}_{t}$ will induce a deformation $\mathcal{E}_{t}$ in $\mathcal{S} \mathfrak{M}_{H}(I, d)$ of $\mathcal{E}$ such that $\mathcal{E}_{t}^{\vee \vee} \cong \mathcal{F}_{i}$. Because $\mathcal{E}_{t \mid 2 C} \cong \mathcal{E}_{\mid 2 C}$ and $\mathcal{E} \notin W^{0}$, by (2) of Proposition 3.6 we know that $\mathcal{E}_{t}$ belongs to $\overline{\mathcal{W}} \cap \mathcal{S M}_{H}(I, d)-W^{0}$. However, since all open strata of $\overline{\mathcal{W}} \cap S \mathfrak{M}_{H}(I, d)$ are contained in $W^{0}, \mathcal{E}_{t}$ must be in $K_{l}$ since $K_{l} \subset \overline{\mathcal{W}} \cap \mathcal{S} \mathfrak{M}_{H}(I, d)-W^{0}$ is open. Thus $\mathcal{F}_{t}$ belong to $Q_{K_{l}}$. Now assume $\mathcal{E}_{\mid C}$ is not locally free. Then we can find deformation $\mathcal{E}_{t}$ of $\mathcal{E}$ such that $\mathcal{E}_{t}^{\vee \vee} \cong \mathcal{F}_{t}$ while $\mathcal{E}_{t \mid C} \cong \mathcal{E}_{\mid C}$. By applying the deformation argument on page 95-98 of [GL] we know that $\mathcal{E}_{t} \in \overline{\mathcal{W}} \cap \mathcal{S} \mathfrak{M}_{H}(I, d)$. Because sheaves in open strata of $\overline{\mathcal{W}} \cap \mathcal{S} \mathfrak{M}_{H}(I, d)$ are locally free along $C, \mathcal{E}_{t}$ must belong to $K_{l}$. Therefore, $\mathcal{F}_{t}$ belong to $Q$. This proves the claim. As a consequence, the dimension of $Q_{K_{l}}$ at $\mathcal{E}^{\vee \vee}$ is identical to the dimension of $Q$ at $\mathcal{E}^{\vee \vee}$. Hence

$\operatorname{codim}\left(Q_{K_{l}}, \mathfrak{M}_{H}(I, d-a)\right) \leq \operatorname{Ext}_{2 C}^{1}\left(\left(\mathcal{E}^{\vee \vee}\right)_{\mid 2 C},\left(\mathcal{E}^{\vee \vee}\right)_{\mid 2 C}\right)^{0} \leq-3 \chi\left(\mathcal{O}_{2 C}\right)+18$, using (3.25). Now let $Q_{0} \subset Q_{K_{l}}$ be the irreducible component containing $\mathcal{E}^{\vee \vee}$. Applying Lemma 1.9, we obtain a non-locally free sheaf $\mathcal{F}_{0} \in \overline{Q_{0}}$ such that $\mathcal{F}_{0}^{\vee \vee} / \mathcal{F}_{0} \cong \mathcal{O}_{p}$ for some $p \in X-C$ in general position. Consequently we can find a sheaf $\mathcal{E}_{0} \in \overline{K_{l}} \subset \mathfrak{M}_{H}(I, d)$ such that $\mathcal{E}_{0} \subset \mathcal{F}_{0}$ and $\mathcal{F}_{0} / \mathcal{E}_{0} \cong \mathcal{O}_{z}$ with $\ell(z)=1$ or 2 and $p \notin \operatorname{supp}(z)$. The key observation is that since $\mathcal{E}_{0}^{\vee \vee} / \mathcal{E}_{0}=\mathcal{O}_{p} \oplus \mathcal{O}_{z}, \mathcal{S} \mathfrak{M}_{H}(I, d)$ at $\mathcal{E}_{0}$ is (analytically) a union of at least two irreducible components. We now choose one such component: Let $p \in U_{p} \subset$ $X-\operatorname{supp}(z)$ be an analytic neighborhood and let $\mathcal{S}_{U_{p}} \mathfrak{M}_{H}(I, d) \subset \mathfrak{S M}_{H}(I, d)$ 
be the set of those $\mathcal{E}$ such that $\mathcal{E}_{\mid U_{p}}$ is not locally free. $\mathcal{S}_{U_{p}} \mathfrak{M}_{H}(I, d)$ is an analytic divisor of $\mathfrak{M}_{H}(I, d)$ smooth at $\mathcal{E}_{0}$, because of (3.24). Now if we let $(A, 0)$ be a versal deformation space of fixed determinant of $\mathcal{E}_{0 \mid 2 C}$, let $\mathcal{U} \subset \mathfrak{M}_{H}(I, d)$ be an analytic neighborhood of $\mathcal{E}_{0}$ and

$$
\varphi:\left(\mathcal{U}, \mathcal{E}_{0}\right) \longrightarrow(A, 0)
$$

be the map provided by Definition 2.3, then the restriction of $\varphi$ to $\mathcal{S}_{U_{p}} \mathfrak{M}_{H}(I, d)$

$$
\varphi^{\prime}:\left(\mathcal{U} \cap \mathcal{S}_{U_{p}} \mathfrak{M}_{H}(I, d), \mathcal{E}_{0}\right) \longrightarrow(A, 0)
$$

will be a submersion, because of (3.24). Because both $\mathfrak{M}_{H}(I, d)$ and $\mathcal{S}_{U_{p}} \mathfrak{M}_{H}(I, d)$ are smooth at $\mathcal{E}_{0}$ and $A$ is smooth at 0 , by shrinking $\mathcal{U}$ if necessarily, we can find a homeomorphism

$$
\Phi: D \times\left(\mathcal{U} \cap \mathcal{S}_{U_{p}} \mathfrak{M}_{H}(I, d)\right) \longrightarrow \mathcal{U},
$$

where $D \subset \mathbb{C}$ is the unit disk, such that after restricting to $\{0\} \times(\mathcal{U} \cap$ $\left.\mathcal{S}_{U_{p}} \mathfrak{M}_{H}(I, d)\right)$, the map $\Phi(0, \cdot)$ is the identity map onto $\mathcal{U} \cap \mathcal{S}_{U_{p}} \mathfrak{M}_{H}(I, d) \subset$ $\mathcal{U}$, and that for each $z \in \mathcal{U} \cap \mathcal{S}_{U_{p}} \mathfrak{M}_{H}(I, d)$ the set $\Phi(D \times\{z\})$ is contained in the fiber $\varphi^{-1}(\varphi(z))$. $\Phi$ induces a deformation retract $\Psi$ defined by $\Psi(r, \Phi(c, z))=\Phi(r c, z)$ for $z \in \mathcal{U} \cap \mathcal{S}_{U_{p}} \mathfrak{M}_{H}(I, d), c \in D$ and $r \in[0,1]$. By (2) of Proposition 3.6, $W^{0}$ and $\overline{\mathcal{W}}$ are preserved under the retraction $\Phi$. Here we have assumed that $\mathcal{U}$ is small enough so that all sheaves in $\mathcal{U}$ are $2 e-H$-stable.

Now we prove that (3.22) and (3.23) have same images. Let $p \in K_{l} \cap \mathcal{U}$ be a general point and let $W \ni p$ be a closed neighborhood of $p$, contained in $\mathcal{U}$, such that $\Delta_{l}=W \cap K_{l}$ is contractible. Let $T_{l}=\Psi([0,1], W)$. Because the retraction $\Psi$ preserves $W^{0}$ and $K_{l} \subset \mathcal{S M}_{H}(I, d)$, the image of (3.22) must be the same as the image of (3.23). Since the former is surjective, (3.23) is surjective as well. This proves that (3.21) is surjective for $i=1$ and 2.

In the remainder of this section, we shall prove the surjectivity of

$$
H_{i}\left(\mathcal{W}_{2}, \mathcal{W}_{2} \cap \mathcal{S} \mathfrak{M}_{H}(I, d)\right) \longrightarrow H_{i}\left(\mathcal{W}_{1}, \mathcal{W}_{1} \cap \mathcal{S M}_{H}(I, d)\right), \quad i \leq 2
$$

We will adapt the same strategy as we did for the surjectivity of (3.21). We still keep the Whitney stratification of $\mathfrak{M}_{H}(I, d)$. Since $\mathcal{W}_{1} \subset \mathfrak{M}_{H}(I, d)$ has pure codimension $g+1$ and is defined by $3 g-2$ equations, and since $\mathfrak{M}_{H}(I, d)$ is a local complete intersection, by Lemma 1.12

$$
H_{i}\left(\mathcal{W}_{1}-T,\left(\mathcal{W}_{1}-T\right) \cap \mathcal{S} \mathfrak{M}_{H}(I, d)\right) \longrightarrow H_{i}\left(\mathcal{W}_{1}, \mathcal{W}_{1} \cap \mathcal{S M}_{H}(I, d)\right), \quad i \leq 2
$$


is surjective, where $T$ is the union of all strata in $B_{2} \subset \mathcal{W}_{1}$ of codimension at least $3 g+1$. Note that $\Lambda_{2}^{C} \cap \mathcal{W}_{1} \subset T$ because $\Lambda_{2}^{C}$ is a union of strata and $\operatorname{codim}\left(\Lambda_{2}^{C}\right) \geq 3 g+2$. Now let

$$
\xi \in H_{i}\left(\mathcal{W}_{1}-T,\left(\mathcal{W}_{1}-T\right) \cap \mathcal{S} \mathfrak{M}_{H}(I, d)\right)
$$

be any element represented by a cycle $(\Sigma, \partial \Sigma)$. Since $\mathcal{W}_{1}-T$ is locally irreducible, by Lemma 1.12 we can assume that $\Sigma \cap B_{2}$ is discrete. Let $\mathcal{E} \in \Sigma \cap B_{2}$ and let $R$ be an irreducible component of $\Lambda_{\mathcal{E}}^{C}$ containing $\mathcal{E}$. Then by Lemma 1.9 we can find a sheaf $\mathcal{E}_{0} \in \Lambda_{\mathcal{E}}^{C}$ such that $\left.\ell_{(}^{\prime} \mathcal{E}_{0}^{\vee \vee} / \mathcal{E}_{0}\right)=$ 1. Because of dimension reason, we can assume $\operatorname{Ext}^{2}\left(\mathcal{E}_{0}, \mathcal{E}_{0}(-2 C)\right)^{0}=0$. Hence $R$ is transversal to $\mathcal{S} \mathfrak{M}_{H}(I, d)$ at $\mathcal{E}_{0}$. Now we pick a differentiable path $\rho:[0,1] \rightarrow R$ connecting $p$ and $\mathcal{E}_{0}$ so that all sheaves $\mathcal{E}^{\prime}$ in $\rho([0,1])$ have vanishing $H^{2}\left(\mathcal{E} n d^{0}\left(\mathcal{E}^{\prime}\right)(-2 C)\right)$. Let $\mathcal{U}$ be a (classical) neighborhood of $\rho([0,1])$ in $\mathfrak{M}_{H}(I, d)$. Without loss of generality, we can assume that there is a tautological family $\mathcal{E}_{\mathcal{U}}$ on $X \times \mathcal{U}$. Now let $0 \in A$ be the versal deformation space of $\mathcal{E}_{\mid 2 C}$ of fixed determinant. According to Definition 2.3, there is an analytic map

$$
\varphi:(\mathcal{U}, \rho([0,1])) \rightarrow(A, 0)
$$

induced by the family $\mathcal{E}_{\mathcal{U}}$, after shrinking $\mathcal{U}$ if necessary. By further shrinking $\mathcal{U}$ if necessary, we can assume $\varphi$ is a submersion and it realizes $\mathcal{U}$ as a product $(-\varepsilon, 1+\varepsilon) \times \mathcal{U}_{0}$ such that $\varphi$ factor through $\varphi_{0}: \mathcal{U}_{0} \rightarrow A$. (Note that $\{t\} \times \mathcal{U}_{0}$ is a normal slice of $\rho([0,1])$ at $\rho(t)$.) Also if we choose the path $\rho([0,1])$ not to tangent to $\mathcal{S M}_{H}(I, d)$ at $\mathcal{E}_{0}=\rho(1)$, we can choose the fiber structure so that $\varphi\left(\{1\} \times \mathcal{U}_{0}\right) \subset \mathcal{S M}_{H}(I, d)$. Therefore we get a deformation retract $\Psi$ of $\varphi\left([0,1] \times \mathcal{U}_{0}\right)$ to $\varphi\left(\{1\} \times \mathcal{U}_{0}\right)$ defined by

$\Psi(r, \varphi(t, z))=\varphi(1-(1-r)(1-t), z), \quad$ for $r \in[0,1], t \in(-\epsilon, 1]$ and $z \in \mathcal{U}_{0}$.

As in the proof of the surjectivity of (3.21), this deformation retract will provide us a new cycle $\left(\Sigma_{1}, \partial \Sigma_{1}\right) \subset\left(\mathcal{W}_{1}-T,\left(\mathcal{W}_{1}-T\right) \cap \mathcal{S M}_{H}(I, d)\right)$ such that $\Sigma_{1} \cap B_{2}$ is one point less than $\Sigma \cap B_{2}$. By repeating of this argument, we eventually get a cycle $\left(\Sigma_{2}, \partial \Sigma_{2}\right) \subset\left(\mathcal{W}_{2}, \mathcal{W}_{2} \cap \mathcal{S} \mathfrak{M}_{H}(I, d)\right)$ that represent the element $\xi$. This proves the surjectivity of (3.27) and thus proves ? Proposition 3.1 .

\section{Proof of the main theorems.}

In this section, we will first prove theorem 0.4 by using Lefschetz hyperplane theorem. After that, we will study the pair $\mathcal{S M}_{d}(H) \subset \mathfrak{M}_{d}(H)$ in detail to establish both theorem 0.5 and 0.1 . 
We shall continue to use the notation developed in the previous sections. For instance, we will denote by $\mathcal{M}_{d}$ the open subset of $\mathfrak{M}_{H}(I, d)$ consisting of all $H$ - $\mu$-stable sheaves $\mathcal{E}$ with $H^{2}\left(\mathcal{E} n d^{0}(\mathcal{E})\right)=0$ and $\mathcal{S} \mathcal{M}_{d}$ the set $\mathcal{M}_{d} \cap$ $\mathcal{S M}_{H}(I, d)$, etc., and $B_{\delta}(p)$ will be the $\delta$-neighborhood of $p$, after fixing an analytic metric of the ambient space. In this section, we will consider only homologies with rational coefficients. To avoid any confusion, for any subset $Z \subset \mathfrak{M}_{H}(I, d)$ we will use the overline, i.e. $\bar{Z}$, to denote the closure of $Z$ in $\mathfrak{M}_{H}(I, d)$ and use $\operatorname{cl}(Z)$ to denote its closure in $\overline{\mathfrak{M}}_{H}(I, d)$. (The only exception is $\overline{\mathfrak{M}}_{H}(I, d)$ that is the moduli of semistable sheaves.) We fix $H_{0}$, $H_{0} \in \mathcal{C} \Subset \mathrm{NS}_{\mathbb{Q}}^{+}$and the $N$ given in lemma 1.3, 1.5 and 1.9. For $d \geq N$, we pick an $\left(H_{0}, I, d\right)$-suitable $H \in \mathcal{C}$.

Recall that by Lemma $1.3, \mathfrak{M}_{H_{0}}(I, d)^{0}$ is birational to $\mathfrak{M}_{H}(I, d)^{0}$, for $d \geq N$. Because the set $B_{0} \subset \mathfrak{M}_{H_{0}}(I, d)^{0}$ and $B \subset \mathfrak{M}_{H}(I, d)^{0}$, where $B$ and $B_{0}$ were introduced in (6) of Lemma 1.3, have codimensions at least 8 and because both $\mathfrak{M}_{H_{0}}(I, d)^{0}$ and $\mathfrak{M}_{H}(I, d)^{0}$ are local complete intersection varieties, by Lemma $1.12 H_{i}\left(\mathfrak{M}_{H_{0}}(I, d)^{0}\right)$ is canonically isomorphic to $H_{i}\left(\mathfrak{M}_{H}\left(I, d^{\prime}\right)^{0}\right)$ and the square

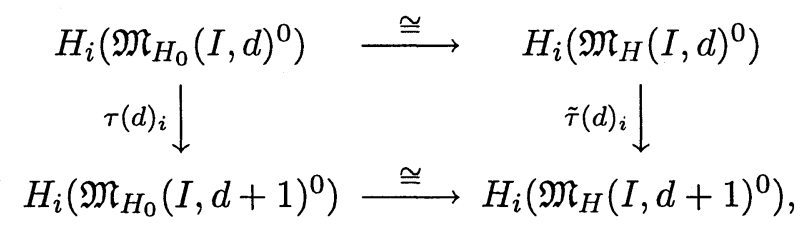

which was introduced in the introduction, is commutative, both for $i \leq 2$. Following the discussion there theorem 0.1 will follow from the surjectivity of $\tau(d)_{i}$, which is equivalent to the surjectivity of $\tilde{\tau}(d)_{i}$.

Theorem 4.1. Let $d \geq N$ and $H \in \mathcal{C}$ be $\left(H_{0}, I, d\right)$-suitable. Then the homomorphism $\tau(d)_{i}: H_{i}\left(\mathfrak{M}_{H}(I, d)^{0}\right) \rightarrow H_{i}\left(\mathfrak{M}_{H}(I, d+1)^{0}\right)$ is surjective for $i \leq 2$.

Before we prove this theorem, let us first fill in the details of the definition of the homomorphism $\tilde{\tau}(d)_{i}$. For any $x \in X$, we let $\mathcal{S}_{1}^{x} \mathcal{M}_{d} \subset \mathcal{S}_{1} \mathcal{M}_{d}$ be the set of $\mathcal{E} \in \mathcal{S}_{1} \mathcal{M}_{d}$ such that $\mathcal{E}$ is not locally free at $x$. Note that $\mathcal{S}_{1}^{x} \mathcal{M}_{d}$ is a $\mathbf{P}^{1}$-bundle over $\mathcal{M}_{d-1}^{0}$, where $\mathcal{M}_{d-1}^{0} \subset \mathcal{M}_{d-1}$ is the open subset of all locally free sheaves. Let $V_{0}$ be a general fiber of this bundle. Then the inclusion 
$V_{0} \subset \mathcal{S}_{1}^{x} \mathcal{M}_{d}$ and the bundle $\mathcal{S}_{1}^{x} \mathcal{M}_{d} \rightarrow \mathcal{M}_{d-1}^{0}$ induce a commutative diagram $(4.1)$

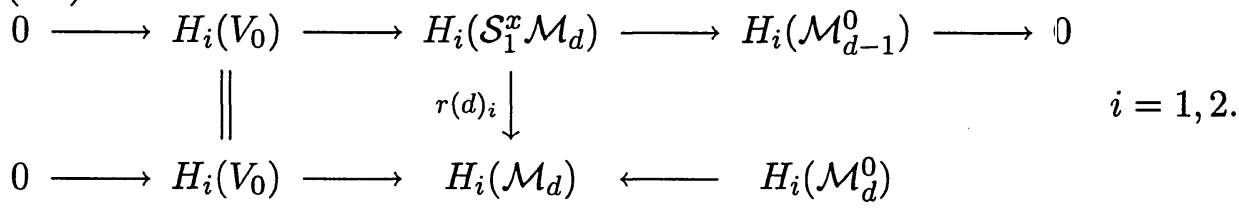

In the following, whenever a space $Z$ admits an obvious map $Z \rightarrow \mathcal{M}_{d}$, we will denote by $H_{i}(Z)^{\mathrm{im}}$ the image of $H_{i}(Z) \rightarrow H_{i}\left(\mathcal{M}_{d}\right)$.

Lemma 4.2. Let $d \geq N$ and $i=1$ or 2 . Then the induced homomorphism $H_{i}\left(\mathcal{M}_{d}^{0}\right) \rightarrow H_{i}\left(\mathcal{M}_{d}\right)$ is injective and its image $H_{i}\left(\mathcal{M}_{d}^{0}\right)^{i m}$ is a complement of $H_{i}\left(V_{0}\right)^{i m}$.

Proof. To show that $H_{i}\left(\mathcal{M}_{d}^{0}\right) \rightarrow H_{i}\left(\mathcal{M}_{d}\right)$ is injective, we need tc show that

$$
H_{i+1}\left(\mathcal{M}_{d}\right) \longrightarrow H_{i+1}\left(\mathcal{M}_{d}, \mathcal{M}_{d}^{0}\right)
$$

is surjective. Let $j=2,3$. Because $\mathcal{M}_{d}$ is smooth, by Lemma 1.13 the above homomorphism is surjective if for any real 1-dimensional subset $T \subset \mathcal{S}_{1} \mathcal{M}_{d}$ the homomorphism

$$
H_{j}\left(\mathcal{M}_{d}^{0} \cup T\right) \longrightarrow H_{j}\left(\mathcal{M}_{d}^{0} \cup T, \mathcal{M}_{d}^{0}\right)
$$

is surjective. Let $T \subset \mathcal{S}_{1} \mathcal{M}_{d}$ be such subset. By perturbing $T$ slightly, we can assume the restriction to $T$ of the map $\mu: \mathcal{S}_{1} \mathcal{M}_{d} \rightarrow X \times \mathcal{M}_{d-1}^{0}$ is one-toone, where $\mu$ sends $\mathcal{E}$ to $\left(\operatorname{supp}\left(\mathcal{E}^{\vee \vee} / \mathcal{E}\right), \mathcal{E}^{\vee \vee}\right)$. Let $V(T)=\mu^{-1}(\mu(T)) . V(T)$ is a $\mathbf{P}^{1}$-bundle over $T$. Now we let $N_{V(T)}$ be a normal slice of $\mathcal{S}_{1} \mathcal{M}_{d} \subset \mathcal{M}_{d}$ along $V(T)$. By the excision of homology,

$$
H_{j}\left(\mathcal{M}_{d}^{0} \cup T, \mathcal{M}_{d}^{0}\right) \cong H_{j}\left(N_{V(T)}, N_{V(T)}-V(T)\right) .
$$

Therefore (4.3) will be surjective if

$$
H_{j}\left(N_{V(T)}\right) \longrightarrow H_{j}\left(N_{V(T)}, N_{V(T)}-V(T)\right)
$$

is surjective. However, after shrinking $N_{V(T)}$ if necessary $N_{V\left(T^{\prime}\right)}$ is a fiber bundle over $T$ with fiber isomorphic to a tubular neighborhood of a $(-l)$ rational curve in a smooth surface, where $l \geq 1$, and that $V^{r}(T)$ is the subbundle whose fiber consists of those $(-l)$-rational curves in the fiber of $N_{V(T)} \rightarrow T$ (see Lemma 1.10). From this description, it is obvious that 
(4.4) is surjective. This proves the the injectivity of $H_{i}\left(\mathcal{M}_{d}^{0}\right) \rightarrow H_{i}\left(\mathcal{M}_{d}\right)$ for $i \leq 2$.

We next show that $H_{i}\left(\mathcal{M}_{d}^{0}\right)^{\mathrm{im}}$ is a complement of $H_{i}\left(V_{0}\right)^{\mathrm{im}}$. Based on the previous argument, it suffices to show that the composition $H_{i}\left(V_{0}\right) \rightarrow$ $H_{i}\left(\mathcal{M}_{d}\right) \rightarrow H_{i}\left(\mathcal{M}_{d}, \mathcal{M}_{d}^{0}\right)$ is an isomorphism. When $i=1$, this is obvious because both $H_{1}\left(V_{0}\right)$ and $H_{1}\left(\mathcal{M}_{d}, \mathcal{M}_{d}^{0}\right)$ are trivial. For $i=2$, we have $H_{2}\left(V_{0}\right)=\mathbb{Q}$ and $H_{2}\left(\mathcal{M}_{d}, \mathcal{M}_{d}^{0}\right) \cong \mathbb{Q}$, since $\mathcal{S M}_{d}$ is an irreducible divisor. The composition $H_{2}\left(V_{0}\right) \rightarrow H_{2}\left(\mathcal{M}_{d}\right) \rightarrow H_{2}\left(\mathcal{M}_{d}, \mathcal{M}_{d}^{0}\right)$ is non-trivial because the intersection number of $V_{0}$ with $\mathcal{S M}_{d}$ in $\mathcal{M}_{d}$ is $-l<0$ (see Lemma 1.10). This completes the proof of the lemma.

We now define the homomorphism

$$
\tau(d-1)_{i}: H_{i}\left(\mathfrak{M}_{H}(I, d-1)^{0}\right) \longrightarrow H_{i}\left(\mathfrak{M}_{H}(I, d)^{0}\right), \quad i \leq 2 .
$$

Let $i=1$ or 2 . Because $H_{i}\left(\mathcal{M}_{d}^{0}\right) \cong H_{i}\left(\mathfrak{M}_{H}(I, d)^{0}\right)$, to construct $\tau(d-1)_{i}$ it suffices to construct a homomorphism

$$
H_{i}\left(\mathcal{M}_{d-1}^{0}\right) \longrightarrow H_{i}\left(\mathcal{M}_{d}^{0}\right) .
$$

Let $f: H_{i}\left(\mathcal{M}_{d}\right) \rightarrow H_{i}\left(\mathcal{M}_{d}^{0}\right)$ be a homomorphism so that $\operatorname{ker}(f)=H_{i}\left(V_{0}\right)^{\text {im }}$ and that the composition $H_{i}\left(\mathcal{M}_{d}^{0}\right) \rightarrow H_{i}\left(\mathcal{M}_{d}\right) \rightarrow H_{i}\left(\mathcal{M}_{d}^{0}\right)$ is the identity. This is possible because $H_{i}\left(\mathcal{M}_{d}^{0}\right) \rightarrow H_{i}\left(\mathcal{M}_{d}\right)$ is injective and $H_{i}\left(\mathcal{M}_{d}^{0}\right)^{\text {im }}$ is a complement of $H_{i}\left(V_{0}\right)^{\mathrm{im}}$. Then using the diagram (4.1) we obtain a homomorphism as in (4.5) that is independent of the choice of $f$. This is the homomorphism mentioned in the introduction, which coincides with the homomorphism $\tau(d-1)_{i}$ introduced by Taubes.

Corollary 4.3. Assume $d \geq N$ and $i \leq 2$, then the homomorphism $\tau(d-$ $1)_{i}$ is surjective if and only if the homomorphism

$$
r(d)_{i}: H_{i}\left(\mathcal{S}_{1}^{x} \mathcal{M}_{d}\right) \longrightarrow H_{i}\left(\mathcal{M}_{d}\right)
$$

is surjective.

The remainder of this section is devoted to prove the surjectivity of (4.6). As explained in the introduction, we shall prove this in two steps: The first step is to use the Lefschetz hyperplane theorem to prove the surjectivity of

$$
H_{i}\left(\mathcal{S M}_{d}\right) \longrightarrow H_{i}\left(\mathcal{M}_{d}\right), \quad i \leq 2 .
$$

The second step is to establish the surjectivity of

$$
r(d)_{i}: H_{i}\left(\mathcal{S}_{1}^{x} \mathcal{M}_{d}\right) \longrightarrow H_{i}\left(\mathcal{M}_{d}\right), \quad i \leq 2
$$


by carefully studying the inclusion $\mathcal{S}_{1}^{x} \mathcal{M}_{d} \subset \mathcal{S M}_{d}$.

We now prove the surjectivity of (4.7). In the following, the index $i$ will take value 1 or 2 unless otherwise is mentioned. We first remark that since $\mathcal{S} \overline{\mathfrak{M}}_{H}(I, d) \subset \overline{\mathfrak{M}}_{H}(I, d)$ is a Cartier divisor and that the singular locus of $\overline{\mathfrak{M}}_{H}(I, d)$ has codimension at least 8 , by Lemma 1.12 for any closed subset $\Lambda \subset \overline{\mathfrak{M}}_{H}(I, d)$ of codimension at least 8

$$
H_{i}\left(\overline{\mathfrak{M}}_{H}(I, d)-\Lambda, \mathcal{S} \overline{\mathfrak{M}}_{H}(I, d)-\Lambda\right) \stackrel{\cong}{\longrightarrow} H_{i}\left(\overline{\mathfrak{M}}_{H}(I, d), \mathcal{S} \overline{\mathfrak{M}}_{H}(I, d)\right) .
$$

In particular $H_{i}\left(\mathcal{M}_{d}, \mathcal{S} \mathcal{M}_{d}\right) \cong H_{i}\left(\overline{\mathfrak{M}}_{H}(I, d), \mathcal{S} \overline{\mathfrak{M}}_{H}(I, d)\right)$. Thus to prove the surjectivity of (4.7) it suffices to prove the vanishing of

$$
H_{i}\left(\overline{\mathfrak{M}}_{H}(I, d), \mathcal{S} \overline{\mathfrak{M}}_{H}(I, d)\right),
$$

which will be accomplished by first proving that

$$
H_{i}\left(\operatorname{cl}(\mathcal{Y}), \operatorname{cl}(\mathcal{Y}) \cap \mathcal{S} \overline{\mathfrak{M}}_{H}(I, d)\right) \longrightarrow H_{i}\left(\overline{\mathfrak{M}}_{H}(I, d), \mathcal{S} \overline{\mathfrak{M}}_{H^{r}}(I, d)\right)
$$

is surjective and then proving

$$
H_{i}\left(\operatorname{cl}(\mathcal{Y}), \operatorname{cl}(\mathcal{Y}) \cap \mathcal{S} \overline{\mathfrak{M}}_{H}(I, d)\right)=0,
$$

where $\mathcal{Y} \subset \mathfrak{M}_{H}(I, d)$ consists of all sheaves $\mathcal{E}$ whose restriction to $C$ is either non-locally free or unstable (See Lemma 1.7). The proof of the surjectivity of (4.10) is based on Lefschetz hyperplane theorem and (4.11) is by applying the vanishing of $H_{i}\left(\overline{\mathcal{W}}, \overline{\mathcal{W}} \cap \mathcal{S} \mathfrak{M}_{H}(I, d)\right)$.

We first prove that (4.10) is surjective. Let

$$
\Psi: \overline{\mathfrak{M}}_{H}(I, d) \longrightarrow \mathbf{P}^{R}
$$

be the morphism constructed in lemma 1.7 and let $V \subset \mathbf{P}^{R}$ be the codimension $3 g-2$ linear subspace such that $\Psi^{-1}(V)=\operatorname{cl}(\mathcal{Y})$. For $\delta>0$, we let $V^{\delta} \subset \mathbf{P}^{R}$ be the $\delta$-neighborhood of $V \subset \mathbf{P}^{R}$ under the Fubini-Study metric and let $\operatorname{cl}(\mathcal{Y})^{\delta}=\Psi^{-1}\left(V^{\delta}\right)$ and $\mathcal{Y}^{\delta}=\Psi^{-1}\left(V^{\delta}\right) \cap \mathfrak{M}_{H}(I, d)$. Since fibers of $\Psi$ can have large dimensions, we shall consider the restriction of $\Psi$ to the open subset $U \subset \mathcal{M}_{d}$ consisting of sheaves $\mathcal{E} \in \mathcal{M}_{d}$ such that $\ell\left(\mathcal{E}^{\vee V} / \mathcal{E}\right) \leq 4$. $U$ is smooth, the compliment of $U$ in $\overline{\mathfrak{M}}_{H}(I, d)$ has codimension at least 5 , and that for any $u \in U$ we have $\operatorname{dim} \Psi^{-1}(\Psi(u)) \leq 15$. The later is true because for any rank 2 locally free sheaf $\mathcal{E}$ the set of subsheaves $\mathcal{F} \subset \mathcal{E}$ with $\ell(\mathcal{E} / \mathcal{F})=5$ has dimension 15 . Then by Lemma 1.12

$$
H_{i}\left(U, U \cap \mathcal{S M}_{d} \cap \mathcal{Y}^{\delta}\right) \stackrel{\cong}{\longrightarrow} H_{i}\left(\mathcal{M}_{d}, \mathcal{S} \mathcal{M}_{d} \cap \mathcal{Y}^{\delta}\right) .
$$


Because the fibers of $\Psi_{\mid U}: U \rightarrow \mathbf{P}^{R}$ have dimension at most 15 and $U$ has pure dimension much bigger than 18, we can apply the stratified Morse theory technique (exactly the same as in the proof of theorem 4.1 on page 195 of $[\mathrm{GM}])$ to the map $\Psi_{\mid U}$ to conclude that

$$
H_{i}\left(U \cap \mathcal{Y}^{\delta}, U \cap \mathcal{S M}_{d} \cap \mathcal{Y}^{\delta}\right) \longrightarrow H_{i}\left(U, U \cap \mathcal{S M}_{d} \cap \mathcal{Y}^{\delta}\right)
$$

is surjective. Then by (4.13),

$$
H_{i}\left(U \cap \mathcal{Y}^{\delta}, U \cap \mathcal{S} \mathcal{M}_{d} \cap \mathcal{Y}^{\delta}\right) \longrightarrow H_{i}\left(\mathcal{M}_{d}, \mathcal{S} \mathcal{M}_{d} \cap \mathcal{Y}^{\delta}\right)
$$

is surjective. Now assuming

$$
H_{i}\left(\mathcal{M}_{d}, \mathcal{S M}_{d} \cap \mathcal{Y}^{\delta}\right) \longrightarrow H_{i}\left(\mathcal{M}_{d}, \mathcal{S M}_{d}\right)
$$

is surjective, then the top line of the following commutative square

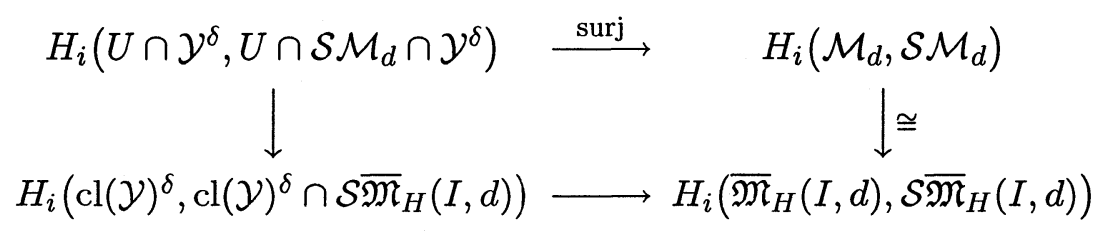

is surjective, then so does the bottom line. Finally, because this surjectivity holds for all $0<\delta \ll 1$, and that $\operatorname{cl}(\mathcal{Y})$ and $\mathcal{S} \overline{\mathfrak{M}}_{H}(I, d)$ are complete, by Proposition 4.A.1 on page 206 of [GM] we obtain the surjectivity of (4.10).

It remains to show that (4.15) is surjective. Note that for $i=0$, it is true because $\mathcal{M}_{d}$ is irreducible and $\mathcal{S} \mathcal{M}_{d}$ is non-empty. We first consider the long exact sequence of homology of triple $\left(\mathcal{S M}_{d} \cap \mathcal{Y}^{\delta}, \mathcal{S} \mathcal{M}_{d}, \mathcal{M}_{d}\right)$ :

$$
H_{i}\left(\mathcal{M}_{d}, \mathcal{S M}_{d} \cap \mathcal{Y}^{\delta}\right) \longrightarrow H_{i}\left(\mathcal{M}_{d}, \mathcal{S M}_{d}\right) \longrightarrow H_{i-1}\left(\mathcal{S M}_{d}, \mathcal{S M}_{d} \cap \mathcal{Y}^{\delta}\right)
$$

Clearly, (4.15) is surjective if $H_{j}\left(\mathcal{S M}_{d}, \mathcal{S M}_{d} \cap \mathcal{Y}^{\delta}\right)=0$ for $j \leq 1$, which we shall prove now. We first claim that for $j \leq 1, H_{j}\left(\mathcal{S} \mathcal{M}_{d}, \mathcal{S}_{1} \mathcal{M}_{d}\right)=0$. $H_{0}=0$ because $\mathcal{S}_{1} \mathcal{M}_{d}$ is dense in $\mathcal{S} \mathcal{M}_{d}$ following from the irreducibility of $\mathcal{M}_{d-1}$. For $H_{1}$, let $f:([0,1], \partial[0,1]) \rightarrow\left(\mathcal{S M}_{d}, \mathcal{S}_{1} \mathcal{M}_{d}\right)$ be a continuous map. Since $\mathcal{S}_{1} \mathcal{M}_{d}$ is dense in $\mathcal{S} \mathcal{M}_{d}$, we can assume without loss of generality that $f^{-1}\left(\mathcal{S} \mathcal{M}_{d}-\mathcal{S}_{1} \mathcal{M}_{d}\right)$ is a finite set, say $\left\{p_{1}, \cdots, p_{k}\right\}$. Because $\mathcal{S} \mathcal{M}_{d}$ is a local complete intersection and the compliment of $\mathcal{S}_{1} \mathcal{M}_{d} \cup \mathcal{S}_{2}^{0} \mathcal{M} \subset \mathcal{S} \mathcal{M}_{d}$ has codimension 2 , by lemma 1.12 we can choose $f$ so that the points $f\left(p_{i}\right)$ are all contained in $\mathcal{S}_{2} \mathcal{M}_{d}$. Let $R_{1}=\mathcal{S}_{2}^{0} \mathcal{M}_{d}$ and let $R_{2}=\mathcal{S}_{2} \mathcal{M}_{d}-\mathcal{S}_{2}^{0} \mathcal{M}_{d}$. Since $R_{1}$ is irreducible and $R_{2}$ is non-empty, by lemma 1.14 we can choose $f$ so that all $f\left(p_{i}\right)$ actually belong to $R_{2}$. On the other hand, because 
$\mathcal{S M}_{d}$ is locally irreducible at $R_{2}$, which will be proved in Lemma 4.7, we can perturb $f$ within $\bigcup\left(B_{\delta}\left(f\left(p_{i}\right)\right) \cap \mathcal{S M}_{d}\right)$ to obtain a representative $f^{\prime}$ of $[f]$ whose image is contained in $\mathcal{S}_{1} \mathcal{M}_{d}$. Therefore, $[f]=0$ and hence $H_{1}\left(\mathcal{S M}_{d}, \mathcal{S}_{1} \mathcal{M}_{d}\right)=0$. Combined with the long exact sequence of homology of triple $\left(\mathcal{S}_{1} \mathcal{M}_{d} \cap \mathcal{Y}^{\delta}, \mathcal{S}_{1} \mathcal{M}_{d}, \mathcal{S} \mathcal{M}_{d}\right)$, we see that

$$
\alpha: H_{1}\left(\mathcal{S}_{1} \mathcal{M}_{d}, \mathcal{S}_{1} \mathcal{M}_{d} \cap \mathcal{Y}^{\delta}\right) \longrightarrow H_{1}\left(\mathcal{S M}_{d}, \mathcal{S}_{1} \mathcal{M}_{d} \cap \mathcal{Y}^{\delta}\right)
$$

is surjective and hence

$$
H_{1}\left(\mathcal{S}_{1} \mathcal{M}_{d}, \mathcal{S}_{1} \mathcal{M}_{d} \cap \mathcal{Y}^{\delta}\right) \rightarrow H_{1}\left(\mathcal{S} \mathcal{M}_{d}, \mathcal{S} \mathcal{M}_{d} \cap \mathcal{Y}^{\delta}\right)
$$

is surjective since $\mathcal{S}_{1} \mathcal{M}_{d} \subset \mathcal{S} \mathcal{M}_{d}$ is dense. Therefore $H_{1}\left(\mathcal{S} \mathcal{M}_{d}, \mathcal{S} \mathcal{M}_{d} \cap \mathcal{Y}^{\delta}\right)=$ 0 if

$$
H_{1}\left(\mathcal{S}_{1} \mathcal{M}_{d}, \mathcal{S}_{1} \mathcal{M}_{d} \cap \mathcal{Y}^{\delta}\right)=0 .
$$

To prove the vanishing of (4.18), we will use the restriction of $\Psi$ in (4.12) to $\mathcal{S}_{1} \mathcal{M}_{d}$ :

$$
\Psi^{\prime}: \mathcal{S}_{1} \mathcal{M}_{d} \longrightarrow \mathbf{P}^{R}
$$

Because fibers of $\Psi^{\prime}$ have dimension 1, we can apply theorem on page 153 of [GM] to conclude that $H_{1}\left(\mathcal{S}_{1} \mathcal{M}_{d}, \mathcal{S}_{1} \mathcal{M}_{d} \cap \mathcal{Y}^{\delta}\right)=0$. Hence $H_{1}\left(\mathcal{S}_{.} \mathcal{M}_{d}, \mathcal{S} \mathcal{M}_{d} \cap\right.$ $\left.\mathcal{Y}^{\delta}\right)=0$. This completes the proof of the surjectivity of (4.10).

We now show that (4.11) is trivial. First, because $H$ is $(I, d)$-generic, the complement of $\mathfrak{M}_{H}(I, d)$ in $\overline{\mathfrak{M}}_{H}(I, d)$ has codimension at least $10 \mathrm{~g}$, because $\mathcal{Y}-\mathcal{S} \mathfrak{M}_{H}(I, d)$ has pure codimension $g+1$ and is defined by $3 g+2$ equations, by Lemma 1.12

$$
H_{i}\left(\mathcal{Y}, \mathcal{Y} \cap \mathcal{S} \mathfrak{M}_{H}(I, d)\right) \longrightarrow H_{i}\left(\operatorname{cl}(\mathcal{Y}), \operatorname{cl}(\mathcal{Y}) \cap \mathcal{S} \overline{\mathfrak{M}}_{H}\left(I, a^{\prime}\right)\right)
$$

is surjective. Let $\mathcal{W} \subset \mathcal{Y}$ be the set of sheaves whose restriction to $C$ is locally free. Since $\mathcal{Y}-\overline{\mathcal{W}} \subset \mathcal{S} \mathfrak{M}_{H}(I, d)$ is open in $\mathcal{Y}$, by excision of homology

$$
H_{i}\left(\overline{\mathcal{W}}, \overline{\mathcal{W}} \cap \mathcal{S} \mathfrak{M}_{H}(I, d)\right) \cong H_{i}\left(\mathcal{Y}, \mathcal{Y} \cap \mathcal{S} \mathfrak{M}_{H}(I, d)\right)
$$

However, we know $H_{i}\left(\overline{\mathcal{W}}, \overline{\mathcal{W}} \cap \mathcal{S} \mathfrak{M}_{H}(I, d)\right)=0$ by Proposition 3.1. Therefore both $H_{i}\left(\mathcal{Y}, \mathcal{Y} \cap \mathcal{S} \mathcal{M}_{d}\right)$ and (4.11) vanish. This proves the vanishing of $H_{i}\left(\mathcal{M}_{d}, \mathcal{S M}_{d}\right)$ and consequently the surjectivity of (4.7).

Our next task is to prove the surjectivity of (4.8). 
Proposition 4.4. Let $H_{0}, H_{0} \in \mathcal{C} \Subset N S_{\mathbb{Q}}^{+}$be fixed. Then for the $N$ given before, $d \geq N$ and $\left(H_{0}, I, d\right)$-suitable $H \in \mathcal{C}$, the homomorphism

$$
r(d)_{i}: H_{i}\left(\mathcal{S}_{1}^{x} \mathcal{M}_{d}\right) \longrightarrow H_{i}\left(\mathcal{M}_{d}\right)
$$

is surjective for $i \leq 2$.

Proof. The statement for $i=0$ follows from [GL,OG1]. The proof that $r(d)_{1}$ is surjective is similar and easier than that of $r(d)_{2}$, which we shall prove in detail. From now on, we assume the proposition is true for $i=1$. We remark that the result of Taubes explained in the introduction and diagram (4.1) implies that if $r(d)_{1}$ is surjective for large $d$, then $\tau(d)_{1}$ is an isomorphism for large $d$. Since $\mathcal{S}_{1}^{x} \mathcal{M}_{d} \rightarrow \mathcal{M}_{d-1}^{0}$ is a $\mathbf{P}^{1}$ bundle, $\tau(d)_{1}$ is an isomorphism for large $d$ implies that $r(d)_{1}$ is an isomorphism for large $d$, which implies further that $H_{1}\left(\mathcal{S}_{2}^{x_{1} x_{2}} \mathcal{M}_{d}\right) \cong H_{1}\left(\mathcal{M}_{d}\right)$. Here $x_{1} \neq x_{2} \in X$ and $\mathcal{S}_{2}^{x_{1} x_{2}} \mathcal{M}_{d}$ consists of $\mathcal{E}$ in $\mathcal{S}_{2}^{0} \mathcal{M}_{d}$ such that $\mathcal{E}^{\vee \vee} / \mathcal{E} \cong \mathcal{O}_{x_{1}} \oplus \mathcal{O}_{x_{2}}$.

We now show the surjectivity of $r(d)_{2}$. Because $H_{2}\left(\mathcal{M}_{d}, \mathcal{S M}_{d}\right)=0$, $H_{2}\left(\mathcal{S M}_{d}\right)^{\mathrm{im}}=H_{2}\left(\mathcal{M}_{d}\right)$. Hence Proposition 4.4 follows from the identities:

$$
H_{2}\left(\mathcal{S} \mathcal{M}_{d}\right)^{\mathrm{im}}=H_{2}\left(\mathcal{S}_{1} \mathcal{M}_{d}\right)^{\mathrm{im}}
$$

and

$$
H_{2}\left(\mathcal{S}_{1} \mathcal{M}_{d}\right)^{\mathrm{im}}=H_{2}\left(\mathcal{S}_{1}^{x} \mathcal{M}_{d}\right)^{\mathrm{im}}
$$

The difficulty in showing the first identity lies in the fact that $\mathcal{S M}_{d}$ is not locally irreducible along $\mathcal{S}_{2}^{0} \mathcal{M}_{d}$. Thus $H_{2}\left(\mathcal{S}_{1} \mathcal{M}_{d}\right) \rightarrow H_{2}\left(\mathcal{S M}_{d}\right)$ is not necessarily surjective. However, we shall show that their images in $H_{2}\left(\mathcal{M}_{d}\right)$ coincide.

To this end, we need to introduce some spaces that will help us understand the geometry of the compliment of $\mathcal{S}_{1} \mathcal{M}_{d} \subset \mathcal{S} \mathcal{M}_{d}$. The first space is $\mathcal{Z}_{1}$ that is an algebraic space whose closed points are pairs

$$
\left\{\mathcal{F}_{1} \subset \mathcal{F}_{2}\right\}: \mathcal{F}_{1} \in \mathcal{S M}_{d} \text { and } \mathcal{F}_{2} \in \mathcal{M}_{d-1} .
$$

We let $\pi_{1}^{\mathcal{Z}_{1}}, \pi_{2}^{\mathcal{Z}_{1}}$ and $\pi_{X}^{\mathcal{Z}_{1}}$ be maps from $\mathcal{Z}_{1}$ to $\mathcal{S} \mathcal{M}_{d}, \mathcal{M}_{d-1}$ and $X$ respectively by sending $\left\{\mathcal{F}_{1} \subset \mathcal{F}_{2}\right\}$ to $\mathcal{F}_{1} \in \mathcal{S} \mathcal{M}_{d}, \mathcal{F}_{2} \in \mathcal{M}_{d-1}$ and $\operatorname{supp}\left(\mathcal{F}_{1} / \mathcal{F}_{2}\right)$ respectively. Note that $\pi_{1}^{\mathcal{Z}_{1}}$ is one-to-one over $\left(\pi_{1}^{\mathcal{Z}_{1}}\right)^{-1}\left(\mathcal{S}_{1} \mathcal{M}_{d}\right)$. The second space we need is the algebraic space consisting of filtrations $\left\{\mathcal{F}_{1} \subset \mathcal{F}_{2} \subset \mathcal{F}_{3}\right\}$ such that $\mathcal{F}_{i} \in \mathcal{M}_{d-i+1}$ and $\ell\left(\mathcal{F}_{i+1} / \mathcal{F}_{i}\right)=1$. We denote this space by $\mathcal{Z}_{2}$. Similarly, we let $\pi_{\mathcal{Z}_{1}}^{\mathcal{Z}_{2}}: \mathcal{Z}_{2} \rightarrow \mathcal{Z}_{1}$ be the map sending $\left\{\mathcal{F}_{1} \subset \mathcal{F}_{2} \subset \mathcal{F}_{3}\right\}$ to 
$\left\{\mathcal{F}_{1} \subset \mathcal{F}_{2}\right\}$ and let $\pi_{i}^{\mathcal{Z}_{2}}: \mathcal{Z}_{2} \rightarrow \mathcal{M}_{d-i+1}$ be the map sending filtrations in $\mathcal{Z}_{2}$ to the i-th term (i.e. $\mathcal{F}_{i}$ ) of the filtrations. $\pi_{X 1}^{\mathcal{Z}_{2}}$ and $\pi_{X 2}^{\mathcal{Z}_{2}}: \mathcal{Z}_{2} \rightarrow X$ are projections that send filtrations to $\operatorname{supp}\left(\mathcal{F}_{2} / \mathcal{F}_{1}\right)$ and $\operatorname{supp}\left(\mathcal{F}_{3} / \mathcal{F}_{2}\right)$ respectively.

Before we proceed, let us first prove four technical lemmas.

Lemma 4.5. Let $\mathcal{S}_{2}^{\prime} \mathcal{M}_{d} \subset \mathcal{S}_{2} \mathcal{M}_{d}-\mathcal{S}_{2}^{0} \mathcal{M}_{d}$ be the subset consisting of $w$ 's so that $\left(\pi_{1}^{\mathcal{Z}_{2}}\right)^{-1}(w) \subset \mathcal{Z}_{2}$ are single point sets. Then

$$
\operatorname{dim}\left(\mathcal{S}_{2} \mathcal{M}_{d}-\mathcal{S}_{2}^{0} \mathcal{M}_{d} \cup \mathcal{S}_{2}^{\prime} \mathcal{M}_{d}\right) \leq \operatorname{dim} \mathcal{M}_{d}-4
$$

Proof. Let $\mathcal{E} \in \mathcal{S}_{2} \mathcal{M}_{d} .\left(\pi_{1}^{\mathcal{Z}_{2}}\right)^{-1}(\mathcal{E})$ is a point if and only if there is a unique filtration

$$
\mathcal{T}_{0} \subset \mathcal{T}_{1} \subset \mathcal{T}_{2}=\mathcal{E}^{\vee \vee} / \mathcal{E}
$$

such that $\ell\left(\mathcal{T}_{j} / \mathcal{T}_{j-1}\right)=1$ for $j=1,2$. When $\mathcal{E} \notin \mathcal{S}_{2}^{0} \mathcal{M}_{d}$, the uniqueness of the above filtration is equivalent to $\left(\mathcal{E}^{\vee \vee} / \mathcal{E}\right) \otimes \mathcal{O}_{p}=\mathcal{O}_{p}$ for some $p \in X$. Hence $\mathcal{S}_{2} \mathcal{M}_{d}-\mathcal{S}_{2}^{0} \mathcal{M}_{d} \cup \mathcal{S}_{2}^{\prime} \mathcal{M}_{d}$ consists of $\mathcal{E}$ such that $\mathcal{E}$ is the kernel of $\mathcal{E}^{\vee \vee} \rightarrow \mathcal{O}_{x}^{\oplus 2}$ for some $x \in X$. Therefore $\mathcal{S}_{2}^{\prime} \mathcal{M}_{d}$ is dense in $\mathcal{S}_{2} \mathcal{M}_{d}-\mathcal{S}_{2}^{0} \mathcal{M}_{d}$ and thus (4.21) follows from $\operatorname{codim}\left(\mathcal{S}_{2} \mathcal{M}_{d}\right)=2$.

Lemma 4.6. $\mathcal{Z}_{1}$ and $\mathcal{Z}_{2}$ are locally irreducible.

Proof. We will prove the second statement and leave the first to the readers. Consider the map $p=\pi_{X 1}^{2} \times \pi_{X 2}^{2} \times \pi_{3}^{\mathcal{Z}_{2}}: \mathcal{Z}_{2} \rightarrow X \times X \times \mathcal{M}_{d-2}$. It is obvious that the fibers of $p$ over $(X \times X-\Delta) \times \mathcal{M}_{d-2}^{0}$ is isomorphic to $\mathbf{P}^{1} \times \mathbf{P}^{1}$, where $\Delta$ is the diagonal in $X \times X$. Since $(X \times X-\Delta) \times \mathcal{M}_{d-1}^{0}$ is smooth, $\mathcal{Z}_{2}$ will be locally irreducible at $\xi \in \mathcal{Z}_{2}$ if for each $\xi^{\prime}$ in an analytic neighborhood of $\xi \in \mathcal{Z}_{2}$ there is a deformation $\xi_{t}^{\prime}$ of $\xi^{\prime}$ in $\mathcal{Z}_{2}$ such that $p\left(\xi_{t}^{\prime}\right) \in(X \times X-\Delta) \times \mathcal{M}_{d-2}^{0}$ for general $t$. Such deformation always exist because of Proposition 6.3 in [Li2] and that $\mathcal{M}_{d-2}^{0} \subset \mathcal{M}_{d-2}$ is clense. This shows that $\mathcal{Z}_{2}$ is locally irreducible.

Lemma 4.7. The algebraic set $\mathcal{S M}_{d}$ is locally irreducible along $\mathcal{S}_{2} \mathcal{M}_{d}-$ $\mathcal{S}_{2}^{0} \mathcal{M}_{d}$.

Proof. Consider $\mathcal{Z}_{1}$ and the projection $\pi_{1}^{\mathcal{Z}_{1}}: \mathcal{Z}_{1} \rightarrow \mathcal{S} \mathcal{M}_{d}$. Clearly, $\pi_{1}^{\mathcal{Z}_{1}}\left(\mathcal{Z}_{1}\right)=$ $\mathcal{S} \mathcal{M}_{d}$. Because fibers of $\pi_{1}^{\mathcal{Z}_{1}}$ over $\mathcal{S}_{2} \mathcal{M}_{d}-\mathcal{S}_{2}^{0} \mathcal{M}_{d}$ are connecte:d, $\mathcal{S} \mathcal{M}_{d}$ is locally irreducible there since $\mathcal{Z}_{1}$ is locally irreducible. This proves of the Lemma. 
Let $\mathcal{S}_{3}^{\prime} \mathcal{M} \subset \mathcal{S}_{3} \mathcal{M}$ be the set of sheaves $\mathcal{E}$ such that $\ell\left(\left(\mathcal{E}^{\vee \vee} / \mathcal{E}\right)_{x}\right) \leq 2$ and $\left(\mathcal{E}^{\vee \vee} / \mathcal{E}\right)_{x} \not \mathcal{O}_{x} \oplus \mathcal{O}_{x}$ for all $x \in X$.

Lemma 4.8. Let $\mathcal{F} \in \mathcal{S}_{2} \mathcal{M}_{d} \cup \mathcal{S}_{3}^{\prime} \mathcal{M}_{d}$ be any sheaf and let $S(\mathcal{F})$ be the set of all sheaves $\mathcal{E}$ such that $\mathcal{E}^{\vee} \cong \mathcal{F}^{\vee}$ and $\ell\left(\left(\mathcal{E}^{\vee \vee} / \mathcal{E}\right)_{x}\right)=\ell\left(\left(\mathcal{F}^{\vee \vee} / \mathcal{F}\right)_{x}\right)$ for each $x \in X$. Then

(1) $H_{i}(S(\mathcal{F}))^{i m} \subset H_{i}\left(V_{0}\right)^{\text {im }}$, where $i=1$ or 2 and $V_{0}=S(\mathcal{E})$ for some $\mathcal{E} \in \mathcal{S}_{1} \mathcal{M}_{d}$

(2) There is a deformation retract neighborhood $U$ of $S(\mathcal{F}) \subset \mathcal{M}_{d}$ such that $H_{1}\left(U-\mathcal{S M}_{d}\right)=0$.

Proof. We first introduce set similar to $\mathcal{Z}_{2}$ that is a desingularization of $S(\mathcal{F})$ as topological space. Let $\mathcal{E}_{0}$ be any sheaf and let $x \in X$ be a point such that $\mathcal{E}_{0}$ is locally free at $x$. We let $R_{1}\left(x, \mathcal{E}_{0}\right)$ be the set of all filtrations $\mathcal{E}_{1} \subset \mathcal{E}_{0}$ such that $\mathcal{E}_{0} / \mathcal{E}_{1} \cong \mathcal{O}_{x}$, and let $R_{2}\left(x, \mathcal{E}_{0}\right)$ be the set of all filtrations $\mathcal{E}_{2} \subset \mathcal{E}_{1} \subset \mathcal{E}_{0}$ such that $\mathcal{E}_{i} / \mathcal{E}_{i+1} \cong \mathcal{O}_{x}$. Let $\pi_{21}: R_{2} \rightarrow R_{1}$ be the map sending $\mathcal{E}_{2} \subset \mathcal{E}_{1} \subset \mathcal{E}_{0}$ to $\mathcal{E}_{1} \subset \mathcal{E}_{0}$. Obviously, $R_{1}\left(x, \mathcal{E}_{0}\right) \cong \mathbf{P}^{1}$ and for any $z \in R_{1}\left(x, \mathcal{E}_{0}\right), \pi_{21}^{-1}(z) \cong \mathbf{P}^{2}$. Thus $R_{2}\left(x, \mathcal{E}_{0}\right)$ is a $\mathbf{P}^{2}$-bundle over $\mathbf{P}^{1}$. Hence, $R_{2}\left(x, \mathcal{E}_{0}\right)$ is simply connected and $H_{2}\left(R_{2}\left(x, \mathcal{E}_{0}\right)\right)=\mathbb{Q}^{\oplus 2}$. Now we prove (1) of the lemma. We will prove the case where $\mathcal{F} \in \mathcal{S}_{2} \mathcal{M}_{d}-\mathcal{S}_{2}^{0} \mathcal{M}_{d}$ and leave the remainder cases to readers. Let $\mathcal{F} \in \mathcal{S}_{2} \mathcal{M}_{d}-\mathcal{S}_{2}^{0} \mathcal{M}_{d}$ and let $x \in X$ be the support of $\mathcal{F}^{\vee \vee} / \mathcal{F}$. Let $\mathcal{E}_{0}=\mathcal{F}^{\vee \vee}$ and let $p: R_{2}\left(x, \mathcal{E}_{0}\right) \rightarrow S(\mathcal{F})$ be the map sending $\mathcal{E}_{2} \subset \mathcal{E}_{1} \subset \mathcal{E}_{0}$ to $\mathcal{E}_{2}$. Then $p$ maps $R_{2}\left(x, \mathcal{E}_{0}\right)$ onto $S(\mathcal{F})$. Let $w \in S(\mathcal{F})$ be the sheaf that is the kernel of $\mathcal{E}_{0} \rightarrow \mathcal{O}_{x}^{\oplus 2}$. Then $p$ is oneto-one away from $Z=p_{2}^{-1}(w)$ and $Z \cong P^{1}$. Hence $H_{1}\left(R_{2}\right) \rightarrow H_{1}(S(\mathcal{F}))$ is an isomorphism and $p_{*}: H_{2}\left(R_{2}\left(x, \mathcal{E}_{0}\right)\right) \rightarrow H_{2}(S(\mathcal{F}))$ is surjective whose kernel contains $[Z]$. Thus $\operatorname{dim} H_{2}(S(\mathcal{F})) \leq 1$. On the other hand, $H_{2}\left(V_{0}\right)^{\text {im }}$ is one dimensional and is obviously contained in $H_{2}(S(\mathcal{F}))^{\mathrm{im}}$. Therefore, $H_{2}(S(\mathcal{F}))^{\mathrm{im}}=H_{2}\left(V_{0}\right)^{\mathrm{im}}$. This proves (1) of the lemma.

Now we prove (2) of the lemma. Let $\mathcal{F} \in \mathcal{S}_{2} \mathcal{M}_{d} \cup \mathcal{S}_{3}^{\prime} \mathcal{M}_{d}$ be any sheaf and let $U$ be a deformation retract neighborhood of $S(\mathcal{F}) \in \mathcal{M}_{d}$. Since $H_{1}(S(\mathcal{F}))=0, H_{1}(U)=0$. Because of the long exact sequence

$$
\longrightarrow H_{2}\left(U, U-\mathcal{S} \mathcal{M}_{d}\right) \longrightarrow H_{1}\left(U-\mathcal{S M}_{d}\right) \longrightarrow H_{1}(U)=0
$$

it suffices to show that $H_{2}\left(U, U-\mathcal{S M}_{d}\right)=0$. Now let $\xi$ be any element in $H_{2}\left(U, U-\mathcal{S M}_{d}\right)$ represented by a cycle $(D, \partial D) \rightarrow\left(U, U-\mathcal{S M}_{d}\right)$. Since $U$ is smooth and since $\operatorname{codim}\left(\mathcal{S}_{2} \mathcal{M}_{d} \cup \mathcal{S}_{3}^{\prime} \mathcal{M}_{d}\right) \geq 2$, we can assume without 
loss of generality that $D \cap \mathcal{S} \mathcal{M}_{d} \subset \mathcal{S}_{1} \mathcal{M}_{d}$. Further, since $S\left(\mathcal{J}^{\mathcal{E}}\right)$ is a deformation retract of $U$, we can assume $D \cap \mathcal{S}_{1} \mathcal{M}_{d}$ is close to $S(\mathcal{F})$ so that if $\left\{\mathcal{F}_{1}, \cdots \mathcal{F}_{k}\right\}=D \cap \mathcal{S}_{1} \mathcal{M}_{d}$ then $S\left(\mathcal{F}_{i}\right) \subset U$. Therefore if we let $U_{i}$ be neighborhood of $S\left(\mathcal{F}_{i}\right) \subset U$, the element $\xi$ will belong to the image of

$$
\oplus^{k} H_{2}\left(U_{i}, U_{i}-\mathcal{S} \mathcal{M}_{d}\right) \longrightarrow H_{2}\left(U, U-\mathcal{S} \mathcal{M}_{d}\right) .
$$

However because $\mathcal{F}_{i} \in \mathcal{S}_{1} \mathcal{M}_{d}$, by Lemma 1.10 we can choose $U_{i}$ so that $H_{1}\left(U_{i}, U_{i}-\mathcal{S} \mathcal{M}_{d}\right)=0$. Therefore $\xi=0$ and hence $H_{1}\left(U-\mathcal{S} \mathcal{M}_{d}\right)=0$. This proves 2) of the lemma.

Now we prove (4.19). Let $\xi \in H_{2}\left(\mathcal{S M}_{d}\right)$ be any element. Since $\mathcal{M}_{d}$ is smooth and $\mathcal{S M}_{d}$ is Cartier, by Lemma 1.12 and 1.13, we can find a Riemann surface $\Sigma$ with $\partial \Sigma=\emptyset$ and a continuous map $f: \Sigma \rightarrow \mathcal{S M}_{d}$ such that the cycle $[f(\Sigma)]$ is a multiple of $\xi$ and that

(4.22): $f(\Sigma) \subset \mathcal{S}_{1} \mathcal{M}_{d} \cup \mathcal{S}_{2}^{0} \mathcal{M}_{d} \cup \mathcal{S}_{2}^{\prime} \mathcal{M}_{d} \cup \mathcal{S}_{3}^{0} \mathcal{M}_{d}, f^{-1}\left(\mathcal{S}_{2}^{0} \mathcal{M}_{d}\right)$ is at most real 1-dimensional and $f^{-1}\left(\mathcal{S}_{2}^{\prime} \mathcal{M}_{d} \cup \mathcal{S}_{3}^{0} \mathcal{M}_{d}\right)$ is discrete.

(Recall $\mathcal{S}_{2}^{\prime} \mathcal{M}$ is defined in Lemma 4.5.) Now let $R=f^{-1}\left(\mathcal{S}_{2}^{0} \mathcal{M}_{d} \cup \mathcal{S}_{2}^{\prime} \mathcal{M}_{d} \cup\right.$ $\left.\mathcal{S}_{3}^{0} \mathcal{M}_{d}\right) . \quad R \subset \Sigma$ is closed. Without loss of generality, we can assume $R$ is piecewise smooth. By our assumption on $f, R$ can be expressed as a disjoint union $R_{1} \cup R_{2}$, where $R_{1}$ is a 1-chain and $R_{2}$ is discrete. We let $S$ be the Riemann surface with boundary obtained by cutting $\Sigma$ along $R_{1}$. Let $\eta: S \rightarrow \Sigma$ be the projection. Because $\pi_{1}^{\mathcal{Z}_{1}}: \mathcal{Z}_{1} \rightarrow \mathcal{S} \mathcal{M}_{d}$ is one-to-one over $f\left(\Sigma-R_{1} \cup R_{2}\right), f_{\mid \Sigma-R_{1} \cup R_{2}}$ lifts to a

$$
g^{\prime}: S-\partial S \cup \eta^{-1}\left(R_{2}\right) \rightarrow \mathcal{Z}_{1} .
$$

$g^{\prime}$ extends to $g: S \rightarrow \mathcal{Z}_{1}$ because $\pi_{1}^{\mathcal{Z}_{1}}$ is finite over $f(\Sigma)$, since (4.22). Next, let $B=\left(\pi_{1}^{\mathcal{Z}_{1}} \circ g\right)^{-1}\left(\mathcal{S}_{3}^{0} \mathcal{M}_{d}\right) \cap \partial S . B$ is discrete and $B$ cuts $\partial S$ into 1-chains $I_{1}, \cdots, I_{k}$. Because each fiber of $\pi_{\mathcal{Z}_{1}}^{\mathcal{Z}_{2}}: \mathcal{Z}_{2} \rightarrow \mathcal{Z}_{1}$ over the interior of $g\left(I_{i}\right)$ consists of single point, we can lift $g_{\mid I_{i}}: I_{i} \rightarrow \mathcal{Z}_{1}$ to $\tilde{g}_{\mid I_{i}}: \coprod_{i} I_{i} \rightarrow \mathcal{Z}_{2}$ such that $g_{\mid I_{i}}=\pi_{\mathcal{Z}_{1}}^{\mathcal{Z}_{2}} \circ \tilde{g}_{\mid I_{i}}$. Let $\amalg I_{i}$ be the disjoint union of $I_{i}$ and $\tilde{g}=\amalg \tilde{g}_{\mid I_{i}}$. Then we have the following diagram:

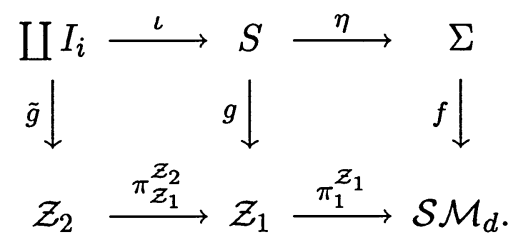

Before going into details, let us first explain the strategy we will use. We will see that Lemma 4.8 implies that (4.19) does hold if we can find a 
representative $f: \Sigma \rightarrow \mathcal{S M}_{d}$ of any $\xi \in H_{2}\left(\mathcal{S M}_{d}\right)$ that satisfies (4.22) and $f^{-1}\left(\mathcal{S}_{2} \mathcal{M}_{d} \cup \mathcal{S}_{3}^{0} \mathcal{M}_{d}\right)$ is discrete. Such choice of $f$ is not obvious since $\mathcal{S} \mathcal{M}_{d}$ is not locally irreducible along the codimension 1 subset $\mathcal{S}_{2}^{0} \mathcal{M}_{d} \subset \mathcal{S} \mathcal{M}_{d}$. Our solution is to utilize the fact that $\mathcal{Z}_{1}$ is locally irreducible. We first find a chain $g(S) \subset \mathcal{Z}_{1}$ covering $f(\Sigma)$ as in (4.23). Since $g(S)$ may not be closed, we need to find a 2-chain $T \subset \mathcal{Z}_{1}$ to close $S$, i.e. $\partial(T+S)=0$. In our case, we will choose a 2-chain $\tilde{T} \subset \mathcal{Z}_{2}$ so that its image $T$ in $\mathcal{Z}_{1}$ will have $\partial T=-\partial S$. This way, we obtain a decomposition

$$
\xi=[g(T+S)]-\left[\pi_{1}^{\mathcal{Z}_{2}}(\tilde{T})\right] .
$$

Since $T+S \subset \mathcal{Z}_{1}$ is closed and $\mathcal{Z}_{1}$ is locally irreducible, we can perturb $T+S$ to a new cycle $A_{1}^{\prime} \subset \mathcal{Z}_{1}$ so that with $A_{1}=\pi_{1}^{\mathcal{Z}_{1}}\left(A_{1}^{\prime}\right),\left[A_{1}\right]=[g(S+T)]$ and $A_{1}-\mathcal{S}_{1} \mathcal{M}_{d}$ is discrete so $\left[A_{1}\right] \in H_{2}\left(\mathcal{S}_{1} \mathcal{M}_{d}\right)^{\text {im }}$ by Lemma 4.8. As to $A_{2}=$ $\pi_{1}^{\mathcal{Z}_{2}}(\tilde{T}) \subset \mathcal{S}_{2} \mathcal{M}_{d} \cup \mathcal{S}_{3}^{\prime} \mathcal{M}_{d}$, by choosing $\tilde{T} \subset \mathcal{Z}_{2}$ generic we can assume $A_{2}-$ $\mathcal{S}_{2}^{0} \mathcal{M}_{d}$ is discrete. Then we can show, by perturbing $A_{2}$ and using Lemma 4.8, that $\left[A_{2}\right] \in H_{2}\left(\mathcal{S}_{2}^{0} \mathcal{M}_{d}\right)^{\mathrm{im}}$. Thus (4.19) follows from $H_{2}\left(\mathcal{S}_{2}^{0} \mathcal{M}_{d}\right)^{\mathrm{im}} \subset$ $H_{2}\left(\mathcal{S}_{1} \mathcal{M}_{d}\right)^{\text {im }}$.

Now we continue our search of the desired representative of $\xi$. We first show how to find the chain $\tilde{T} \subset \mathcal{Z}_{2}$ such that $\pi_{\mathcal{Z}_{1}}^{\mathcal{Z}_{2}}(\tilde{T})+S$ is closed. Since $\iota\left(\amalg I_{i}\right) \subset S$ is a closed chain, $\iota\left(\amalg \partial I_{i}\right)=0$. Then we can index $\amalg \partial I_{i}$ to be $p_{1}^{+}, p_{1}^{-}, \ldots, p_{m}^{+}, p_{m}^{-}$such that $\iota\left(p_{j}^{+}\right)=\iota\left(p_{j}^{-}\right)=p_{j} \in \partial S$. We claim that for each $j$ we can find a path $A_{j} \subset \mathcal{Z}_{2}$ such that $\partial A_{j}=-p_{j}^{+}+p_{j}^{-}$and $\pi_{\mathcal{Z}_{1}}^{\mathcal{Z}_{2}}\left(A_{j}\right)=0$ as chain. Indeed, since $\left(\pi_{1}^{\mathcal{Z}_{1}} \circ g\right)\left(p_{j}\right) \in \mathcal{S}_{3}^{0} \mathcal{M}_{d}$, it associates to a sheaf $\mathcal{E}$ such that $\mathcal{E}$ is the kernel of $\mathcal{E}^{\vee \vee} \rightarrow \mathcal{O}_{x_{0}} \oplus \mathcal{O}_{x_{+}} \oplus \mathcal{O}_{x_{-}}$. By rearranging the index of $x_{*}$ 's, we can assume $g\left(p_{j}\right)$ corresponds to the filtration $\left\{\mathcal{E} \subset \mathcal{E}_{1}\right\}$, where $\mathcal{E}_{1} / \mathcal{E} \cong \mathcal{O}_{x_{0}}$, and $\tilde{g}\left(p_{j}^{ \pm}\right)$corresponds to the filtration $\left\{\mathcal{E} \subset \mathcal{E}_{1} \subset \mathcal{E}_{ \pm}\right\}$, where $\mathcal{E}_{1} / \mathcal{E}_{ \pm} \cong \mathcal{O}_{x_{ \pm}}$. We now fix a smooth path $\rho_{j}:[0,1] \rightarrow X$ connecting $x_{-}$and $x_{+}$that is contained in a smooth affine curve $D \subset X$ disjoint from $x_{0}$. We fix a trivialization $\left(\mathcal{E}_{1}^{\vee \vee}\right)_{\mid D} \cong \mathcal{O}_{D} \oplus \mathcal{O}_{D}$ such that $\mathcal{E}_{1}$ is the kernel of

$$
\mathcal{E}_{1}^{\vee \vee} \rightarrow\left(\mathcal{E}_{1}^{\vee \vee}\right)_{\mid D} \cong \mathcal{O}_{D} \oplus \mathcal{O}_{D} \stackrel{\mathrm{pr}_{1}}{\longrightarrow} \mathcal{O}_{D} \rightarrow \mathcal{O}_{x_{+}} \oplus \mathcal{O}_{x_{-}} .
$$

We then let $\psi_{j}:[0,1] \times[0,1] \rightarrow D \times D$ be the map sending $(r, s)$ to $\left(\rho_{j}(r), \rho_{j}(s)\right)$. Associated to each $w=\left(w_{1}, w_{2}\right) \in D \times D$ we have a filtration of sheaves

$$
[\mathcal{F}]_{w}=\left\{\mathcal{F}_{1, w} \subset \mathcal{F}_{2, w} \subset \mathcal{F}_{3, w}\right\} \in \mathcal{Z}_{2},
$$

where $\mathcal{F}_{3, w}$ is the kernel of the surjective composite

$$
\mathcal{E}_{1}^{\vee \vee} \longrightarrow\left(\mathcal{E}_{1}^{\vee \vee}\right)_{\mid D} \stackrel{\mathrm{pr}_{1}}{\longrightarrow} \mathcal{O}_{D} \longrightarrow \mathcal{O}_{w_{1}}
$$


$\mathcal{F}_{2, w}$ is the kernel of the above surjective composite with $\mathcal{O}_{w_{1}}$ replaced by $\mathcal{O}_{\pi(w)}$, where $\pi(w) \subset D$ is the subscheme corresponding to the image of $w$ via $\pi: D \times D \rightarrow \operatorname{Hilb}^{2}(D)$ and $\mathcal{F}_{1, w}$ is the sheaf so that $\mathcal{F}_{1, w} \rightarrow \mathcal{F}_{2, w}$ is isomorphic to $\mathcal{E} \rightarrow \mathcal{E}_{1}$ away from $D$. (i.e. $\mathcal{F}_{2, w} / \mathcal{F}_{1, w} \cong \mathcal{O}_{x_{0}}$.) We denote the map $w \mapsto[\mathcal{F}]_{w}$ by $\varphi_{j}$, where $w \in D \times D$. Now let $J$ be the anti-diagonal: $\{r+s=1\} \subset[0,1] \times[0,1]$ and let $A_{j}=\varphi_{j} \circ \psi_{j}(J) \subset \mathcal{Z}_{2} . A_{j}$ is a 1-chain with $\partial A_{j}=-p_{j}^{+}+p_{j}^{-}$, with appropriate orientation. Since $\pi(J)=0$ as 1-chain in $\operatorname{Hilb}^{2}(D), \pi_{\mathcal{Z}_{1}}^{\mathcal{Z}_{2}}\left(A_{j}\right)=0$ as chain. Therefore

$$
\Gamma=\sum_{i=1}^{k} \tilde{g}\left(I_{i}\right)+\sum_{j=1}^{m} A_{j} \subset \mathcal{Z}_{2}
$$

is closed and as chain $\pi_{\mathcal{Z}_{1}}^{\mathcal{Z}_{2}}(\Gamma)=g(\partial S)$.

Lemma 4.9. There is a 2-chain $T \subset \mathcal{Z}_{2}$ such that $\partial T=-\Gamma$, that $\pi_{1}^{\mathcal{Z}_{2}}(T) \subset \mathcal{S}_{2} \mathcal{M}_{d} \cup \mathcal{S}_{3}^{\prime} \mathcal{M}_{d}$ and $\pi_{1}^{\mathcal{Z}_{2}}(T) \cap \mathcal{S}_{3}^{0} \mathcal{M}_{d}$ is discrete.

Proof. As chains,

$$
\partial\left(\pi_{X}^{\mathcal{Z}_{1}} \circ g(S)\right)=\pi_{X}^{\mathcal{Z}_{1}} \circ g(\partial S)=\pi_{X}^{\mathcal{Z}_{1}} \circ \pi_{\mathcal{Z}_{1}}^{\mathcal{Z}_{2}}(\Gamma) .
$$

Thus $\pi_{X}^{\mathcal{Z}_{1}} \circ \pi_{\mathcal{Z}_{1}}^{\mathcal{Z}_{2}}(\Gamma)$ represents the trivial class in $H_{1}(X)$. Beca,use $\pi_{X 1}^{\mathcal{Z}_{2}}=$ $\pi_{X}^{\mathcal{Z}_{1}} \circ \pi_{\mathcal{Z}_{1}}^{\mathcal{Z}_{2}},\left[\pi_{X 1}^{\mathcal{Z}_{2}}(\Gamma)\right]=0$ in $H_{1}(X)$. We claim $\left[\pi_{X 2}^{\mathcal{Z}_{2}}(\Gamma)\right]$ is also trivial in $H_{1}(X)$. To prove this we first rearrange indices of $I_{1}, \cdots, I_{k}$ to $I_{1}^{ \pm}, \cdots, I_{l}^{ \pm}$ such that $\eta\left(I_{i}^{+}\right)=-\eta\left(I_{i}^{-}\right)$. This is possible because $\eta(\partial S)=0$, at least after we subdivide those chains having $\eta\left(I_{i}\right)=0$. Next, we let $\Lambda_{1}$ be those $I_{i}^{ \pm}$ such that $\operatorname{Im} g\left(I_{i}^{+}\right)=\operatorname{Im} g\left(I_{i}^{-}\right)$(which means that we do not need to cut $\Sigma$ along $I_{i}$ in order to get the lifting $g$ ) and let $\Lambda_{2}$ be the remainder $I_{i}$ 's. Note that for $i \in \Lambda_{1}, g\left(I_{i}^{+}\right)=-g\left(I_{i}^{-}\right)$, which implies $\tilde{g}\left(I_{i}^{+}\right)=-\tilde{g}\left(I_{i}^{-}\right)$. Then we have the following identities of chains:

$$
\begin{gathered}
\pi_{X 2}^{\mathcal{Z}_{2}} \circ \tilde{g}\left(I_{i}^{+}\right)+\pi_{X 2}^{\mathcal{Z}_{2}} \circ \tilde{g}\left(I_{i}^{-}\right)=0, \quad \text { if } I_{i}^{ \pm} \in \Lambda_{1} ; \\
\pi_{X 1}^{\mathcal{Z}_{2}} \circ g\left(I_{i}^{ \pm}\right)=-\pi_{X 2}^{\mathcal{Z}_{2}} \circ \tilde{g}\left(I_{i}^{\mp}\right), \quad \text { if } I_{i}^{ \pm} \in \Lambda_{2} .
\end{gathered}
$$

The second identity holds because for any $z \in \mathcal{S}_{2}^{0} \mathcal{M}_{d}$ and $\left(\pi_{1}^{\mathcal{Z}_{2}}\right)^{-1}(z)=$ $\left\{\xi_{1}, \xi_{2}\right\}$ we have $\pi_{X 1}^{\mathcal{Z}_{2}}\left(\xi_{1}\right)=\pi_{X 2}^{\mathcal{Z}_{2}}\left(\xi_{2}\right)$. As to the 1-chains $A_{1}, \cdots, A_{m}$ we added on, the class

$$
\left[\pi_{X 2}^{\mathcal{Z}_{2}}\left(\sum_{j=1}^{m} A_{j}\right)\right] \in H_{1}(X)
$$


certainly depends on the choices of paths $\rho_{j}$. However, since path $\rho_{j}$ can be chosen arbitrary so long as its end points are as prescribed, we can assume without loss of generality that (4.26) is indeed trivial. Therefore we have

$$
\left[\pi_{X 2}^{\mathcal{Z}_{2}}(\Gamma)\right]=-\left[\pi_{X 1}^{\mathcal{Z}_{2}}(\Gamma)\right]=0 \in H_{1}(X) .
$$

Now we consider the projection $\pi_{3}^{\mathcal{Z}_{2}}: \mathcal{Z}_{2} \rightarrow \mathcal{M}_{d-2}$ that sends $\mathcal{F}_{1} \subset \mathcal{F}_{2} \subset$ $\mathcal{F}_{3}$ to $\mathcal{F}_{3}$. Because $\mathcal{M}_{d-2}$ is smooth and $\mathcal{Z}_{2}$ is locally irreducible, we can perturb $\Gamma$ to $\Gamma^{\prime} \subset \mathcal{Z}_{2}$ such that $\pi_{3}^{\mathcal{Z}_{2}}\left(\Gamma^{\prime}\right) \subset \mathcal{M}_{d-2}^{0}$. Let $T_{1}$ be the 2-chain in $\mathcal{Z}_{2}$ so that $\partial T_{1}=\Gamma^{\prime}-\Gamma$. By choosing $\Gamma^{\prime}$ close to $\Gamma$ and $T_{1}$ in general position, we can make $T_{1}-\Gamma \subset\left(\pi_{1}^{\mathcal{Z}_{2}}\right)^{-1}\left(\mathcal{S}_{2}^{0} \mathcal{M}_{d}\right)$ since $\Gamma \subset\left(\pi_{1}^{\mathcal{Z}_{2}}\right)^{-1}\left(\mathcal{S}_{2} \mathcal{M}_{d} \cup \mathcal{S}_{3}^{\prime} \mathcal{M}_{d}\right)$ and $\mathcal{Z}_{2}$ is locally irreducible. (See proof of Lemma 1.13.) Let $\mathcal{X}=\left(\pi_{3}^{Z_{2}}\right)^{-1}\left(\mathcal{M}_{d-2}^{0}\right)$. It is easy to check that fibers of $\mathcal{X}$ over $\mathcal{M}_{d-2}^{0} \times X \times X\left(\right.$ via $\left.\pi_{3}^{\mathcal{Z}_{2}} \times \pi_{X 1}^{\mathcal{Z}_{2}} \times \pi_{X 2}^{\mathcal{Z}_{2}}\right)$ are connected and have trivial first homology groups. By Leray spectral sequence, $H_{1}(\mathcal{X})$ is isomorphic to $H_{1}\left(\mathcal{M}_{d-2}^{0} \times X \times X\right)$. Because of (4.27), for any pair of distinct points $\left(x_{1}, x_{2}\right) \in X \times X$ there is a 1-cycle $\Gamma^{\prime \prime}$ contained in

$$
\left(\pi_{X 1}^{\mathcal{Z}_{2}} \times \pi_{X 2}^{\mathcal{Z}_{2}}\right)^{-1}\left(x_{1}, x_{2}\right) \subset \mathcal{X} \subset \mathcal{Z}_{2}
$$

such that $\Gamma^{\prime \prime}$ is homologous to $\Gamma^{\prime}$ in $\mathcal{X}$, via $T_{2} \subset \mathcal{X}$. Because $\pi_{1}^{\mathcal{Z}_{2}}\left(\Gamma^{\prime \prime}\right) \subset$ $\mathcal{S}_{2}^{x_{1} x_{2}} \mathcal{M}_{d}$ and $\pi_{1}^{\mathcal{Z}_{2}}\left(\Gamma^{\prime \prime}\right)$ is the boundary of $\pi_{1}^{\mathcal{Z}_{2}}\left(T_{1}+T_{2}\right) \subset \mathcal{S} \mathcal{M}_{d}$ (note that $\pi_{1}^{\mathcal{Z}_{1}}(g(\Gamma))=0$ as chain $),\left[\pi_{1}^{\mathcal{Z}_{2}}\left(\Gamma^{\prime \prime}\right)\right] \in H_{1}\left(\mathcal{S}_{2}^{x_{1} x_{2}} \mathcal{M}_{d}\right)$ is contained in the kernel of $h: H_{1}\left(\mathcal{S}_{2}^{x_{1} x_{2}} \mathcal{M}_{d}\right) \rightarrow H_{1}\left(\mathcal{M}_{d}\right)$. By the induction hypothesis, $h$ is an isomorphism, hence $\left[\pi_{1}^{\mathcal{Z}_{2}}\left(\Gamma^{\prime \prime}\right)\right]=0 \in H_{1}\left(\mathcal{S}_{2}^{x_{1} x_{2}} \mathcal{M}_{d}\right)$, and hence $\left[\Gamma^{\prime \prime}\right]=0 \in H_{1}\left(\left(\pi_{1}^{\mathcal{Z}_{2}}\right)^{-1}\left(\mathcal{S}_{2}^{x_{1} x_{2}} \mathcal{M}_{d}\right)\right)$. Therefore, we can find a 2-chain $T_{3} \subset\left(\pi_{1}^{\mathcal{Z}_{2}}\right)^{-1}\left(\mathcal{S}_{2}^{x_{1} x_{2}} \mathcal{M}_{d}\right)$ such that $\partial T=-\Gamma^{\prime \prime}$. By construction, the 2chain $T=T_{1}+T_{2}+T_{3}$ satisfies the condition required in the lemma, since $\pi_{1}^{\mathcal{Z}_{2}}(\mathcal{X}) \subset \mathcal{S}_{2} \mathcal{M}_{d}$. This completes the proof of the lemma.

Continuation of the proof of (4.19). Let $T \subset \mathcal{Z}_{2}$ be the chain just constructed. We let $B_{1}=g(S)+\pi_{\mathcal{Z}_{1}}^{\mathcal{Z}_{2}}(T)$ and $B_{2}=\pi_{1}^{\mathcal{Z}_{2}}(T)$, as chains in $\mathcal{Z}_{1}$ and $\mathcal{S}_{2} \mathcal{M}_{d} \cup \mathcal{S}_{3}^{\prime} \mathcal{M}_{d}$ respectively. By our construction, $\partial B_{1}=0$, $\pi_{1}^{\mathcal{Z}_{1}}\left(B_{1}\right) \subset \mathcal{S}_{1} \mathcal{M}_{d} \cup \mathcal{S}_{2} \mathcal{M}_{d} \cup \mathcal{S}_{3}^{\prime} \mathcal{M}_{d}$ and $\pi_{1}^{\mathcal{Z}_{1}}\left(B_{1}\right)-\mathcal{S}_{1} \mathcal{M}_{d} \cup \mathcal{S}_{2}^{0} \mathcal{M}_{d}$ is discrete. For $B_{2}$, we also have $\partial B_{2}=0$ and that $B_{2}-\mathcal{S}_{2} \mathcal{M}_{d} \subset \mathcal{S}_{3}^{\prime} \mathcal{M}_{d}$ is discrete. Let $u_{1}$ be the image of $\left[B_{1}\right]$ under $H_{2}\left(\mathcal{Z}_{1}\right) \rightarrow H_{2}\left(\mathcal{S M}_{d}\right)$ and $u_{2}$ be $\left[B_{2}\right] \in H_{2}\left(\mathcal{S} \mathcal{M}_{d}\right)$. Clearly, the cycle $\xi \in H_{2}\left(\mathcal{S M}_{d}\right)$ we begin with has a decomposition $\xi=u_{1}+u_{2}$.

We now show that $u_{1}^{\mathrm{im}} \in H_{2}\left(\mathcal{S}_{1} \mathcal{M}_{d}\right)^{\mathrm{im}}$ and $u_{2}^{\mathrm{im}} \in H_{2}\left(\mathcal{S}_{1}^{x} \mathcal{M}_{d}\right)^{\mathrm{im}} \subset$ $H_{2}\left(\mathcal{S}_{1} \mathcal{M}_{d}\right)$, where $u_{i}^{\mathrm{im}}$ is the image of $u_{i}$ in $H_{2}\left(\mathcal{M}_{d}\right)$. We first prove $u_{1}^{\mathrm{im}} \in H_{2}\left(\mathcal{S}_{1} \mathcal{M}_{d}\right)^{\mathrm{im}}$. We take the representative $B_{1} \subset \mathcal{Z}_{1}$. By perturbing $B_{1} \subset \mathcal{Z}_{1}$, we can assume $\pi_{1}^{\mathcal{Z}_{1}}\left(B_{1}\right)-\mathcal{S}_{1} \mathcal{M}_{d}$ is finite and is contained in 
$\mathcal{S}_{2} \mathcal{M}_{d} \cup \mathcal{S}_{3}^{\prime} \mathcal{M}_{d}$, since $\mathcal{Z}_{1}$ is locally irreducible. Now consider the projection

$$
\pi=\pi_{X}^{\mathcal{Z}_{1}} \times \pi_{2}^{\mathcal{Z}_{1}}: \mathcal{Z}_{1} \longrightarrow X \times \mathcal{M}_{d-1}
$$

Clearly, $\pi\left(B_{1}\right)-X \times \mathcal{M}_{d-1}^{0}$ is discrete. Let $\left\{\left(x_{1}, \mathcal{F}_{1}\right), \cdots,\left(x_{l}, \mathcal{F}_{l}\right)\right\}=\pi\left(B_{1}\right)-$ $X \times \mathcal{M}_{d-1}^{0}$ and let $S\left(\mathcal{F}_{i}\right) \subset \mathcal{S} \mathcal{M}_{d-1}$ be the closed subset defined before. (i.e. $S\left(\mathcal{F}_{i}\right)$ consists of $\mathcal{E}$ such that $\mathcal{E}^{\vee} \cong \mathcal{F}_{i}^{\vee}$ and $\ell\left(\left(\mathcal{E}^{\vee \vee} / \mathcal{E}\right)_{x}\right)=\ell\left(\left(\mathcal{F}_{i}^{\vee \vee} / \mathcal{F}_{i}\right)_{x}\right)$ for each $x \in X$.) By Lemma 4.8, there is a deformation retract neighborhood $U_{i}$ of $\left\{x_{i}\right\} \times S\left(\mathcal{F}_{i}\right) \subset X \times \mathcal{M}_{d-1}$ such that $H_{1}\left(U_{i}-X \times \mathcal{S M}_{d-1}\right)=0$. Therefore by Mayer-Vietoris sequence

$$
\left[B_{1}\right] \in H_{2}\left(\pi^{-1}\left(X \times \mathcal{M}_{d-1}^{0}\right)\right) \oplus \oplus^{l} H_{2}\left(\pi^{-1}\left(U_{i}\right)\right)
$$

and hence

$$
u_{1}^{\mathrm{im}} \in H_{2}\left(\mathcal{S}_{1} \mathcal{M}_{d}\right)^{\mathrm{im}} \oplus \oplus^{l} H_{2}\left(\pi_{1}^{\mathcal{Z}_{1}}\left(\pi^{-1}\left(U_{i}\right)\right)\right)^{\mathrm{im}} .
$$

Since $U_{i}$ is a deformation retract of $\left\{x_{i}\right\} \times S\left(\mathcal{F}_{i}\right)$ and $\mathcal{Z}_{1} \rightarrow X \times \mathcal{M}_{d-1}$ is proper,

$$
H_{2}\left(\pi_{1}^{\mathcal{Z}_{1}}\left(\pi^{-1}\left(U_{i}\right)\right)\right)^{\mathrm{im}}=H_{2}\left(\pi_{1}^{\mathcal{Z}_{1}}\left(\pi^{-1}\left(\left\{x_{i}\right\} \times S\left(\mathcal{F}_{i}\right)\right)\right)\right)^{\mathrm{im}} .
$$

However, $\pi_{1}^{\mathcal{Z}_{1}}\left(\pi^{-1}\left(\left\{x_{i}\right\} \times S\left(\mathcal{F}_{i}\right)\right)\right)$ is exactly $S\left(\tilde{\mathcal{F}}_{i}\right)$ for any $\tilde{\mathcal{F}}_{i}$ in this set. Therefore because $\tilde{\mathcal{F}}_{i} \in \mathcal{S}_{2} \mathcal{M}_{d} \cup \mathcal{S}_{3}^{\prime} \mathcal{M}_{d}$ and Lemma 4.8 , the right hand side of (4.28) is contained in $H_{2}\left(\mathcal{S}_{1} \mathcal{M}_{d}\right)^{\mathrm{im}}$. This shows that $u_{1}^{\mathrm{im}} \in H_{1}\left(\mathcal{S}_{1} \mathcal{M}_{d}\right)^{\mathrm{im}}$.

Next, we study the class $u_{2}$. To do this, we need to introduce a new set similar to $\mathcal{Z}_{1}$. We let $\mathcal{Z}_{3}$ be the set of filtrations $\mathcal{F}_{1} \subset \mathcal{F}_{2}$ such that $\mathcal{F}_{1} \in \mathcal{S}_{2} \mathcal{M}_{d} \cup \mathcal{S}_{3}^{\prime} \mathcal{M}_{d}$ and $\ell\left(\mathcal{F}_{2} / \mathcal{F}_{1}\right)=2$. The obvious map $\pi_{1}^{\mathcal{Z}_{3}}$ sending any filtration to its first factor is a map from $\mathcal{Z}_{3}$ to $\mathcal{S}_{2} \mathcal{M}_{d} \cup \mathcal{S}_{3}^{\prime} \mathcal{M}_{d}$. $\pi_{1}^{\mathcal{Z}_{3}}$ is finite over $\mathcal{S}_{2} \mathcal{M}_{d} \cup \mathcal{S}_{3}^{\prime} \mathcal{M}_{d}$, and is one-to-one over $\mathcal{S}_{2} \mathcal{M}_{d}$. Let $B_{2} \subset \mathcal{S}_{2} \mathcal{M} 1_{d} \cup \mathcal{S}_{3}^{0} \mathcal{M}_{d}$ be the closed chain representing $u_{2}$. We know $B_{2} \cap \mathcal{S}_{3}^{\prime} \mathcal{M}_{d}$ is finite. Therefore because $\pi_{1}^{\mathcal{Z}_{3}}$ is finite, which is true based on our choice of $\mathcal{S}_{3}^{\prime} \cdot \mathcal{M}_{d}$ before Lemma 4.8, we can find a closed 2-chain $\tilde{B}_{2} \subset \mathcal{Z}_{3}$ such that $\pi_{1}^{\mathcal{Z}_{3}}\left(\tilde{B}_{2}\right)=B_{2}$. Finally, because $\left(\pi_{1}^{\mathcal{Z}_{3}}\right)^{-1}\left(\mathcal{S}_{2}^{0} \mathcal{M}_{d}\right)$ is locally irreducible, which can be proved similar to that of $\mathcal{Z}_{1}$, we can perturb $B_{2}$ so that $\pi_{1}^{\mathcal{Z}_{3}}\left(B_{2}\right)-\mathcal{S}_{2}^{0} \mathcal{M}_{d}$ is finite. We now show $u_{2} \in H_{2}\left(\mathcal{S}_{1} \mathcal{M}_{d}\right)^{\text {im }}$. Let

$$
\pi: \mathcal{Z}_{3} \longrightarrow S^{2} X \times \mathcal{M}_{d-2}
$$

be the map sending $\mathcal{F}_{1} \subset \mathcal{F}_{2}$ to $\left\{\operatorname{supp} \mathcal{F}_{2} / \mathcal{F}_{1}, \mathcal{F}_{2}\right\}$. By Lemma 4.8 , fibers of $\pi$ over $S^{2} X \times \mathcal{M}_{d-1}^{0}$ are simply connected. Hence similar to the discussion concerning (4.27),

$$
u_{2}^{\mathrm{im}}=\left[B_{2}\right]^{\mathrm{im}} \in H_{2}\left(\pi^{-1}\left(\left(S^{2} X-\Delta\right) \times \mathcal{M}_{d-2}^{0}\right)\right)^{\mathrm{im}} \oplus\left(\oplus H_{2}\left(S\left(\mathcal{F}_{i}^{-}\right)\right)^{\mathrm{im}}\right),
$$


where the summation runs over all possible $\mathcal{F}_{i} \in \pi_{1}^{\mathcal{Z}_{3}}\left(B_{2}\right)-\mathcal{S}_{2}^{0} \mathcal{M}_{d}$. By Lemma 4.8, the last factor is contained in $H_{2}\left(\mathcal{S}_{1} \mathcal{M}_{d}\right)^{\mathrm{im}}$. Because $\pi^{-1}\left(\left(S^{2} X-\Delta\right) \times \mathcal{M}_{d-2}^{0}\right)=\mathcal{S}_{2}^{0} \mathcal{M}_{d}, u_{2}^{\mathrm{im}}$ will belong to $H_{2}\left(\mathcal{S}_{1} \mathcal{M}_{d}\right)^{\mathrm{im}}$ if $H_{2}\left(\mathcal{S}_{2}^{0} \mathcal{M}_{d}\right)^{\mathrm{im}} \subset H_{2}\left(\mathcal{S}_{1} \mathcal{M}_{d}\right)^{\mathrm{im}}$.

In the following, we will show

$$
H_{2}\left(\mathcal{S}_{1} \mathcal{M}_{d}\right)^{\mathrm{im}} \subset H_{2}\left(\mathcal{S}_{1}^{x} \mathcal{M}_{d}\right)^{\mathrm{im}} \text { and } H_{2}\left(\mathcal{S}_{2}^{0} \mathcal{M}_{d}\right)^{\mathrm{im}} \subset H_{2}\left(\mathcal{S}_{1}^{x} \mathcal{M}_{d}\right)^{\mathrm{im}} .
$$

In particular this will imply that both $u_{1}^{\mathrm{im}}$ and $u_{2}^{\mathrm{im}}$ and then $\xi^{\mathrm{im}}$ are in $H_{2}\left(\mathcal{S}_{1}^{x} \mathcal{M}\right)^{\mathrm{im}}$. We will prove the first inclusion and leave the proof of the second inclusion to the readers. We need to show that for any $\eta \in H_{2}\left(\mathcal{S}_{1} \mathcal{M}_{d}\right)$, we can find $\xi \in H_{2}\left(\mathcal{S}_{1}^{x} \mathcal{M}_{d}\right)$ such that $\eta^{\mathrm{im}}=\xi^{\mathrm{im}}$ in $H_{2}\left(\mathcal{M}_{d}\right)$. Since $\mathcal{S}_{1} \mathcal{M}_{d}$ is a $\mathbf{P}^{1}$-bundle over $X \times \mathcal{M}_{d-1}^{0}$ with projection $\pi$, we have the following commutative diagram (of exact sequences)

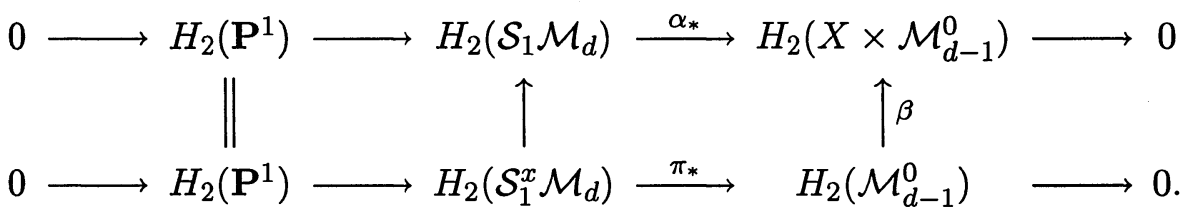

Clearly, $\beta$ factor through $H_{0}(X) \otimes H_{2}\left(\mathcal{M}_{d-1}^{0}\right) \subset H_{2}\left(X \times \mathcal{M}_{d-1}^{0}\right)$, according to the Kunneth decomposition

$$
H_{2}\left(X \times \mathcal{M}_{d-1}\right) \doteq H_{2}\left(\mathcal{M}_{d-1}^{0}\right) \oplus H_{1}(X) \otimes H_{1}\left(\mathcal{M}_{d-1}^{0}\right) \oplus H_{2}(X) .
$$

Now we consider the case where $\alpha_{*}(\eta) \in H_{1}(X) \otimes H_{1}\left(\mathcal{M}_{d-1}^{0}\right)$. We choose a ball $B \subset X$ containing $x$ and a compact $S \subset \mathcal{M}_{d-1}^{0}$ such that

$$
\alpha_{*}^{-1}\left(H_{1}(X) \otimes H_{1}\left(\mathcal{M}_{d-1}^{0}\right)\right)^{\mathrm{im}} \subset H_{2}\left(\mathcal{W}_{S}\right)^{\mathrm{im}},
$$

where $\mathcal{W}_{S}$ is the set of $\mathcal{E} \in \mathcal{S}_{1} \mathcal{M}_{d}$ such that $\mathcal{E}$ is a subsheaf of some $\mathcal{F} \in S$ such that $\operatorname{supp}(\mathcal{F} / \mathcal{E}) \cap B=\emptyset$. Because $H_{1}\left(\mathcal{S}_{1}^{x} \mathcal{M}_{d-1}\right) \rightarrow H_{1}\left(\mathcal{M}_{d-1}\right)$ is an isomorphism, by induction hypothesis, we can replace $S$ by a set $S^{\prime} \subset$ $\mathcal{S}_{1}^{x} \mathcal{M}_{d-1}$ and still have (4.31) with $S$ replaced by $S^{\prime}$. Note that $\mathcal{W}_{S^{\prime}} \subset$ $\mathcal{S}_{2}^{0} \mathcal{M}_{d}$. Now let $\mathcal{S}_{2}^{x} \mathcal{M}_{d}$ be the set of all sheaves $\mathcal{E} \in \mathcal{S}_{2}^{0} \mathcal{M}_{d}$ such that $\ell\left(\left(\mathcal{E}^{\vee \vee} / \mathcal{E}\right)_{x}\right)=1$. Then $\mathcal{S}_{2}^{x} \mathcal{M}_{d}$ is a smooth divisor of $\mathcal{S}_{1}^{x} \mathcal{M}_{d} \cup \mathcal{S}_{2}^{x} \mathcal{M}_{d}$, and further for any $w \in \mathcal{S}_{2}^{x} \mathcal{M}_{d}$ there is a neighborhood $U$ of $w \in \mathcal{S}_{1}^{x} \mathcal{M}_{d} \cup \mathcal{S}_{2}^{x} \mathcal{M}_{d}$ such that $H_{1}\left(U-\mathcal{S}_{2}^{x} \mathcal{M}_{d}\right)=0$, following the proof of Lemma 1.10. Therefore similar to the argument after (4.28)

$$
H_{2}\left(\mathcal{W}_{S^{\prime}}\right)^{\mathrm{im}} \subset H_{2}\left(\mathcal{S}_{1}^{x} \mathcal{M}\right)^{\mathrm{im}} \oplus H_{2}\left(V_{0}\right)^{\mathrm{im}} \subset H_{2}\left(\mathcal{S}_{1}^{x} \mathcal{M}_{d}\right)^{\mathrm{im}} .
$$


Therefore the left hand side of (4.31) is contained in $H_{2}\left(\mathcal{S}_{1}^{x} \mathcal{M}_{d}\right)^{\mathrm{im}}$. The case where $\alpha_{*}^{-1}\left(H_{2}(X)\right)^{\mathrm{im}} \subset H_{2}\left(\mathcal{S}_{1}^{x} \mathcal{M}_{d}\right)^{\mathrm{im}}$ can be proved similarly. We will omit the proof here. This proves (4.19) and (4.20).

Now we are ready to prove the main theorems. Let $H_{0}$ be any ample divisor, let $H_{0} \in \mathcal{C} \Subset \mathrm{NS}_{\mathbb{Q}}^{+}$be a precompact neighborhood of $H_{0} \Subset \mathrm{NS}_{\mathbb{Q}}^{+}$and let $N$ be a sufficiently large constant given before. Then for any $H \in \mathcal{C}$ and $d \geq N, H_{i}\left(\mathfrak{M}_{H}(I, d)^{0}\right)$ is isomorphic to $H_{i}\left(\mathfrak{M}_{H_{0}}(I, d)^{0}\right)(i \leq 2$ here and in the later discussion). However, for $d \geq N$ and $\left(H_{0}, I, d\right)$-suitable $H \in \mathcal{C}$, by theorem 4.1

$$
H_{i}\left(\mathfrak{M}_{H}(I, d)^{0}, \mathbb{Q}\right) \longrightarrow H_{i}\left(\mathfrak{M}_{H}(I, d+1)^{0}, \mathbb{Q}\right)
$$

is surjective. Therefore,

$$
H_{i}\left(\mathfrak{M}_{H_{0}}(I, d)^{0}, \mathbb{Q}\right) \longrightarrow H_{i}\left(\mathfrak{M}_{H_{0}}(I, d+1)^{0}, \mathbb{Q}\right)
$$

is surjective for all $d \geq N$. Since $H_{i}\left(\mathfrak{M}_{H_{0}}(I, d)^{0}\right)$ are linear spaces, the above chain of homomorphisms has to stabilize at finite steps. Namely, for some $N_{1} \geq N$, it is an isomorphism for all $d \geq N_{1}$. Further, combined with the work of [Ta] (see (0.3) and (0.4)), we can find $N_{2}$ so that for $d \geq N_{2}$,

$$
H_{i}\left(\mathfrak{M}_{H_{0}}(I, d)^{0}, \mathbb{Q}\right) \cong H_{i}\left(\mathcal{B}\left(P_{d}\right)^{*}, \mathbb{Q}\right) .
$$

This proves theorem 0.1. For theorem 0.2 , we simply apply the above isomorphism to the fact

$$
\operatorname{dim} H_{1}\left(\mathcal{B}\left(P_{d}\right)^{*}\right)=b_{1} \text { and } \operatorname{dim} H_{2}\left(\mathcal{B}\left(P_{d}\right)^{*}\right)=b_{2}+\frac{1}{2} b_{1}\left(b_{1}-1\right),
$$

where $b_{i}=\operatorname{dim} H_{i}(X)$ (see page 181-182 of [DK] $]^{4}$ ). Finally, by the proof of lemma $4.3, h_{1}\left(\mathfrak{M}_{H_{0}}(I, d)\right)=h_{1}\left(\mathfrak{M}_{H_{0}}(I, d)^{0}\right)$, and $h_{2}\left(\mathfrak{M}_{H_{1}}(I, d)\right)=$ $h_{2}\left(\mathfrak{M}_{H_{0}}(I, d)^{0}\right)+1$ because $\mathcal{S} \mathfrak{M}_{H_{0}}(I, d) \subset \mathfrak{M}_{H_{0}}(I, d)$ is an irreducible Cartier divisor. This proves theorem 0.3 .

\section{References.}

[At] I. V. Artamkin, On deformation of sheaves, Math. USSR, Izv. 32 (no. 3) (1989), 663-668.

\footnotetext{
${ }^{4}$ Since $\mathrm{SU}(2)$ or $\mathrm{SO}(3)$ is a rational three sphere, the first two rational homology groups of $\tilde{\mathcal{B}}^{*}$ and $\mathcal{B}^{*}$ coincide.
} 
[Ar] M. Artin, Algebraic approximation of structures over complete local rings, Publ. Math. IHES. 36 (1969), 23-58.

[AJ] M.F. Atiyah and J.D. Jones, Topological aspects of Yang-Mills theory, Comm. Math. Phy. 61 (1978), 97-118.

[Be] A. Beauville, Sur la cohomologie de certains espaces de modules de fibrés vectoriels, preprint.

$\left[\mathrm{BHM}^{2}\right]$ C.P. Boyer, J.C. Hurtubise, B.M. Mann and R.J. Milgram, The topology of instanton moduli spaces. I: The Atiyah-Jones conjecture, Ann of Math. 137 (1993), 561-609.

[Do] S.K. Donaldson, Polynomial invariants for smooth four-manifolds, Topology, 29 (No. 3) (1986), 257-315.

[DK] S.K. Donaldson and P.B. Kronheimer, The Geometry of Four-Manifolds, Oxford Mathematical Monographs, Oxford Science Publications, 1990.

[DN] J.M. Drezet and S. Narasimhan, Group de Picard des varietes de modules de fibres semi-stables sur les coubes algebriques, Invent. Math. 97 (1989), 53-94.

[ES] G. Ellingsud and S.A.Strømme, Toward the Chow ring of the Hilbert scheme of $\mathbf{P}^{2}$, J. Reine. Angew. Math. 441 (1993), 33-44.

[Fr] R. Friedman, Vector bundles over surfaces, to be published.

[GH] L. Göttsche and D. Huybrechts, Hodge numbers of moduli spaces of stable bundles on K3 surfaces, preprint.

[GL] D. Gieseker and J. Li, E, Irreducibility of moduli of rank two vector bundles, J. Diff. Geom. 40 (1994), 23-104.

[GM] M. Goresky and R. MacPherson, Stratified Morse Theory, Ergebnisse der Mathematik und ihrer Grenzgebiete, Springer-Verlag 3 Band 14, 1988.

[Ha] H.A. Hamm, Lefschetz theorems for singular varieties, Proceeding of Symposia in Pure Mathematics, 40 (1983), 547-557.

[HM] J.C. Hurtubise and R.J. Milgram, The Atiyah-Jones conjecture for ruled surfaces, preprint, 1994.

[Ki] F. Kirwan, Geometric invariant theory and Atiyah-Jones conjecture, preprint, 1993.

[La] R. Lazarsfeld, Some applications of the theory of positive vector bundles, Lecture Notes in Mathematics 1092 (1984), 29-61. 
[Li1] J. Li, Algebraic geometric interpretation of Donaldson's polynomial invariants, J. of Diff. Geometry, 37 (1993), 417-466.

[Li2] J. Li, Kodaira dimension of moduli space of vector bundles on surfaces, Invent. Math. 115 (1994), 1-40.

[Li3] J. Li, The Picard group of moduli space of stable sheaves over algebraic surfaces, to appear in Taniguchi Symposium on Mathematics.

[Ma] M. Maruyama, Moduli of stable sheaves, II, J. Math. Kyoto Univ. 18-3 (1978), 557-614.

[Mu] D. Mumford, Towards an enumerative geometry of the moduli space of curves, Arithmetic and Geometry, Volume II, Progress in Mathematics, Birkhauser Boston, Inc. 1983.

[OG1] K. O'Grady, The irreducible components of moduli spaces of vector bundles on surfaces, Invent. Math. 112 (1993), 586-613.

[OG2] K. O'Grady, Moduli of vector bundles on projective surfaces: some basic results, Invent. Math. 123 (no. 1) (1996), 141-207.

[Qi] Z. Qin, Birational properties of moduli spaces of stable locally free rank-2 sheaves on algebraic surfaces, Manuscripta Math. 72 (1991), 163-180.

[Ta] C. Taubes, The stable topology of self-dual moduli spaces, J. Diff. Geom. 19 (1984), 337-392.

[T1] Y-L. Tian, The based SU(n)-instanton moduli spaces, Math. Ann. 298 (1994), 117-139.

[T2] Y-L. Tian, The Atiyah-Jones conjecture for classical group.s, preprint, 1994.

[Yo] K. Yoshioka, The Betti numbers of the moduli space of stable sheaves of rank 2 on $\mathbf{P}^{2}$, J. reine angew. Math. 453 (1994), 193-220.

[Zh] K. Zhu, Generic smoothness of the moduli of rank two stable bundles over an algebraic surface, 207 (No.4) (1991), 629-643.

RECEIVED MAY 14, 1996.

DePARTMENT OF MATHEMATiCS

STANFORD UNIVERSITY

STANFORD, CA 94305

E-mail address: JLI@GAUSS.STANFORD.EDU 